Journal of Patient-Centered

\title{
Abstracts From the 2021 Health Care Systems Research Network Annual Conference
}

Follow this and additional works at: https://aah.org/jpcrr

Part of the Diseases Commons, Health and Medical Administration Commons, Medical Specialties Commons, Mental and Social Health Commons, and the Public Health Commons

\section{Recommended Citation}

Abstracts from the 2021 Health Care Systems Research Network Annual Conference. J Patient Cent Res Rev. 2021;8:154-217. doi: 10.17294/2330-0698.1882

Published quarterly by Midwest-based health system Advocate Aurora Health and indexed in PubMed Central, the Journal of Patient-Centered Research and Reviews (JPCRR) is an open access, peer-reviewed medical journal focused on disseminating scholarly works devoted to improving patient-centered care practices, health outcomes, and the patient experience. 


\title{
Abstracts From the 2021 Health Care Systems Research Network Annual Conference
}

\begin{abstract}
The Health Care Systems Research Network (HCSRN) is comprised of 19 not-forprofit health systems with embedded research departments. The network's annual conference serves as a forum for member institutions to highlight research findings, explore new collaborations, and share insights and opportunities. Abstracts accepted for oral and poster presentation at the 2021 HCSRN Conference are published in this supplement of Journal of Patient-Centered Research and Reviews, the official scientific journal of the annual conference. J Patient Cent Res Rev. 2021;8:154-217.
\end{abstract}

\section{ADDICTION SCIENCE/SUBSTANCE USE}

\section{Does the Change in Smoking and Marijuana Use Policies Influence Adolescents' Risky Sexual Behaviors and Other Associated Factors?}

Ruaa Al Juboori, Orlando Davy, Thembekile Shato, Divya Subramaniam, Qiang Fu

\section{Saint Louis University / SSM Health, St. Louis, MO}

Background: Changing policies around marijuana use and the increase in the use of e-cigarettes have affected patterns of risky sexual behaviors (RSBs) among adolescents. Despite a decline in some RSBs like condom non-use in previous years, few researchers have analyzed how adolescents' RSB trends have been influenced by other risky behaviors such as smoking, marijuana use, fighting, and binge drinking, which is the objective of the current study.

Methods: A total of 61,072 students from 4 Youth Risk Behavior Surveillance System survey years (1993, 2001, 2009, and 2017) were included. The distributions for each outcome within each risk behavior were examined to assess whether a trend existed. Multivariate logistic regression models were constructed to examine the relationship between 5 risky behaviors (suicide risk, smoking, binge drinking, marijuana use, and physical fight) and 2 indicators of RSBs (condom non-use and multiple sexual partners). Interactions between survey year and risky behavior were used to assess whether changes in the patterns of behavior across survey years were either widening or narrowing. An odds ratio of $>1$ implied divergence in the strength of the behaviors from previous years, whereas an odds ratio of $<1$ implied the strength of associations were converging to a current year with respect to the base year 1993.

Results: Although there were significant associations between risky behaviors like suicidality and RSBs, a narrowing in the trend of the association between some risky behaviors and RSBs was noticed. There was a convergence in the trend of association between fighting in 2009 , and binge drinking in 2001, with condom non-use as compared to 1993 . Also, there was a similar pattern between fighting and suicidal risk in 2017 as compared to 1993 for multiple sexual partners. However, there were no consistent decreasing changes in associations across time in marijuana use and smoking with RSBs.

Conclusion: Further longitudinal studies are needed to clarify directionality and policy recommendations for substance use as proxy to reduce the growing burden of sexually transmitted infections among the adolescent population.

Association Between Pharmacotherapy for Opioid Use Disorder and Counseling in OfficeBased Versus Specialty Substance Use Disorder Treatment Settings

Richard Grucza, Ned Presnall, Kevin Xu, Carrie Mintz

\section{Saint Louis University / SSM Health, St. Louis, MO}

Background: Expanding pharmacotherapy for opioid use disorder (OUD) in the United States is a high priority for public health officials. Buprenorphine, a partial opioid agonist, was approved by the U.S. Food and Drug Association in 2002 for the maintenance treatment of OUD. Office-based opioid therapy with buprenorphine expanded rapidly, but specialty substance use disorder (SSUD) settings have been slower to universally adopt buprenorphine therapy. To our knowledge, no literature has directly reported the likelihood of receiving medication in these two settings among people in treatment for OUD. The analyses presented here examine the association between receipt of counseling services for OUD and receipt of medication in both office-based and SSUD treatment settings. 
Methods: We identified 234,804 Medicaid- and commercially insured individuals in the IBM MarketScan database who utilized OUD counseling services from 2006 to 2016. For these individuals, we identified pharmacy claims for buprenorphine, oral naltrexone, and extended-release naltrexone and used the "days supply" variable to identify co-prescription of OUD pharmacotherapy on counseling days. We identified 34.1 counseling service days per individual, on average. Individuals averaged 34.1 counseling service days. Using procedure codes to differentiate officebased individual and group psychotherapy (CPT codes) from comparable services in SSUD settings (HCPCS-H codes), we examined the association between counseling services and pharmacotherapy in each setting. We examined 3 outcomes: 1) coverage with any medication on counseling days; 2) coverage with buprenorphine on counseling days; and 3) coverage with naltrexone (oral or extended-release) on counseling days.

Results: Depending on the type of counseling received (individual, group-based, or intensive), patients had 2.7 to 4.2 times higher odds of being treated with medication on counseling days in office-based vs SSUD settings. Odds ratios for buprenorphine receipt ranged from 3.7 to 7.3 for office-based vs SSUD settings. In contrast, naltrexone was more likely to used be in SSUD settings (odds ratios ranging from 0.3 to 0.5 for office-based vs SSUD).

Conclusion: The disparity in pharmacotherapy practices between office-based and SSUD care is large and consistent with documented biases against agonist OUD pharmacotherapy in SSUD settings.

\section{Medication Storage and Disposal Among Opioid- Naïve Patients in Primary Care}

Mia Lussier, Lorraine Tusing, Jove Graham, Alanna Kulchak Rahm, Christina Gregor, Eric Wright

\section{Geisinger Research, Forty Fort, PA}

Background: Prescription opioids in the home are a potential contributor to opioid-related deaths. Proper opioid storage and disposal is known to reduce medication misuse. Prior studies supporting safe storage and disposal practices largely focus on postsurgery patients, demonstrating improved storage and disposal with education and supply of a disposal mechanism. We aimed to implement and evaluate a safe medication storage and disposal program for patients newly prescribed opioid medications in primary care. Herein, we report baseline storage and disposal practices among patients newly prescribed opioids in primary care.

Methods: We developed a best practice alert for patients newly prescribed an opioid medication across 12 primary care locations. The initial best practice alert was silent, allowing us to determine baseline storage and disposal patterns. A telephone survey was conducted among all eligible subjects 30-45 days after encounter. The survey was adapted from previously used surveys and included storage and disposal questions targeted at the prescribed opioid medication. Household information, such as the presence of children in the home, someone living with chronic pain, health literacy, education, and income, were collected in order to evaluate factors that may influence storage and disposal behaviors.

Results: Of 307 eligible participants, 208 (68\%) completed the survey; 47 participants $(23 \%)$ indicated that their doctor talked to them about disposal, and $60(29 \%)$ indicated that their doctor talked to them about storage. Of the orders placed, 71 (34\%) were written for CII medications, and 203 (96\%) were written for as-needed use; 14 participants (7\%) did not fill their prescription, and $145(70 \%)$ had medication remaining at the time of the survey. Four participants (2\%) reported their medication was stored in a locked location. Among those who had leftover opioid, 13 participants (8\%) disposed of their medication, of whom 5 (38\%) did so by a Centers for Disease Control and Prevention-recommended method.

Conclusion: In a survey of participants without any intervention to improve storage and disposal methods, we found few stored medications safely or disposed leftover medications properly. Future analysis will evaluate how interventions (ie, active best practice alert, education, reminder call, and disposal bag mailed to home) affected participants' storage and disposal of opioid medications.

\section{CANCER}

\section{Treatment Decision-Making in Multiple Myeloma: A Pilot Study}

Mara Epstein, Joann Wagner, Jill Zitzewitz, Kathleen M. Mazor

\section{Meyers Primary Care Institute, Worcester, MA}

Background: There is no single standard of care for treating multiple myeloma (MM). The choice of potential treatment options, including autologous stem cell transplantation and newer, less intensive therapies, makes it important to understand how patients with MM are informed about treatment options. In this pilot study, we examined patients' perceptions of treatment decisions and the factors they considered important when selecting first-line treatment for MM.

Methods: We developed a 60-item questionnaire combining an existing measure of communication in cancer care with new items. Participants were recruited through ResearchMatch, an online, volunteer-based research platform, and additionally through a local multiple myeloma support group. Responses were scored on a Likert scale, with 
some open-ended questions. Eligible participants were men and women 18-80 years of age who had been previously diagnosed with MM.

Results: 12 participants (50\% male gender; 92\% White race; median age: 65.5 years) responded to the survey, and $75 \%$ were diagnosed with MM more than 3 years prior. Twothirds received chemotherapy as primary treatment, and 50\% had an autologous stem cell transplantation. All participants reported having a provider available to answer questions soon after diagnosis. However, 4 participants $(33 \%)$ disagreed with the statement "I knew what would happen next, and what decisions I would face" after diagnosis, suggesting communication gaps. Comments included "I wasn't given much information about my prognosis" and "could have explained more fully the benefits and drawbacks of each treatment." The majority (83\%) reported "always" being involved in treatment decisions, and 92\% understood why decisions were made. However, only 42\% "always" understood the risks of different treatment choices. Patients sought information on $\mathrm{MM}$ from varied and multiple sources, including their doctor $(75 \%)$, the internet $(67 \%)$, nurses $(42 \%)$, cancer societies $(42 \%)$, and friends/family (17\%). Participants identified "overall survival" (50\%), and "cost" $(25 \%)$ as the most important factors in treatment decisions.

Conclusion: Most MM patients responding to this pilot survey were involved in treatment decision-making, although the risks and benefits of each treatment were not always well understood. These preliminary results reveal gaps in patientprovider communication around MM treatment and a need to more fully elucidate barriers to ensure all MM patients receive care that aligns with their goals and preferences.

\section{Determinants of Urologists' Adherence to Active Surveillance Follow-Up Guideline for Low-Risk Prostate Cancer}

Soohyun Hwang, Sarah Birken

\section{University of North Carolina at Chapel Hill, Chapel Hill, NC}

Background: The National Comprehensive Cancer Network (NCCN) guidelines offer guidance for follow-up care for patients with low-risk prostate cancer on active surveillance. However, in practice, $70 \%$ of patients receive follow-up care that is not guideline-adherent, characterized by insufficient or excessive surveillance testing, potentially diminishing active surveillance effectiveness and contributing to poor patient outcomes. The objective of this study is to identify provider- and organization-level determinants of guidelineadherent active surveillance follow-up care.

Methods: We used in-depth semi-structured qualitative interviews with 12 U.S.-based urologists to examine determinants of urologists' adherence to the active surveillance follow-up guideline. Guided by the combined use of the Consolidated Framework for Implementation Research, which focuses on organization-level determinants, and the Theoretical Domains Framework, which focuses on provider-level determinants, we used template analysis to identify multilevel determinants of urologists' adherence to guideline-recommended active surveillance follow-up care. Results: Relevant determinants were comfort with varied utilization behaviors of the guideline, perspectives on the prostate biopsy procedure, and the degree of structure within the practice setting. At the provider level, there was variation in how urologists provided active surveillance follow-up care. All urologists referred to the NCCN guidelines; however, most urologists adapted the guidelines to their needs and/or comfort level (eg, following a subset of recommendations, adapting the interval/frequency of serial tests). Most providers felt that strictly adhering to the repeated biopsy aspect of the guideline was difficult because of concerns about fitting everybody to one type of frequency that does not stratify patients by risk. Others reflected on patients expressing physical discomfort and concerns of infection stemming from the biopsy procedure. At the organization level, urologists in a structured practice environment had the tendency toward providing NCCN guideline-adherent care, whereas urologists practicing in settings with less organization relied more on individual discretion, which created room for flexibility with the care that they provide.

Conclusion: Both provider- and organization-level determinants affected urologists' provision of follow-up care, which may partially explain why patients eventually fail to receive NCCN guideline-adherent active surveillance follow-up care. Findings call on the need for multilevel strategies to increase adherence or to modify existing guidelines to reflect the need at multiple levels.

\section{Electronic Patient-Reported Outcome Measures in Head and Neck Cancer}

Jeewanjot Grewal, Theresa Zatirka, Amy Williams, Steven Chang, Samantha Tam

\section{Henry Ford Health System, Detroit, MI}

Background: Electronic patient-reported outcome measures (ePROMs) represent a patient-centric essential representation of the patient experience. While traditional assessments have relied on provider-generated data, these data correlate poorly with ePROMs as indicators of outcome. Implementation of ePROMs alone in a medical oncology setting has improved the length of systemic treatment and overall survival, decreased acute care visits and emergency department visits, and improved patient-reported quality of life. Thus far, the application of ePROMs has mainly been limited to clinical trial settings. This study presents 
preliminary results from patients with head and neck cancer in a systemwide implementation of ePROMs.

Methods: The institution implemented ePROMs throughout the health system, including the multidisciplinary head and neck surgical/medical/radiation oncology clinics, beginning in fall 2020. The National Institutes of Health's validated and standardized Patient-Reported Outcomes Measurement Information System (PROMIS) was utilized to capture 4 core oncology domains: fatigue, pain interference, physical function, and depression. ePROMs were recorded before, during, and after treatment. Questionnaires were administered prior to patient appointments via MyChart, and an iPad was given to the patients at the time of check-in should the questionnaires be incomplete before each visit.

Results: In all, 106 patients successfully completed PROMIS questionnaires from September to October 2020. Mean PROMIS scores were 51.4 (standard deviation [SD]: 8.8) for the depression scale, 52.4 (SD: 10.0) for fatigue, 56.7 (SD: 10.4) for pain, and 43.7 (SD: 9.6) for physical function. Physical function scores were the only subscale to vary significantly within the cohort. Patients scored 0.22 points less on the physical function subscale with each year of increasing age $(\mathrm{P}=0.004)$. As well, patients with oropharyngeal primaries had significantly higher physical function subscores (mean: 48.7, 95\% CI: 44.9-52.5) compared to patients with laryngeal primaries (mean: 39.7, 95\% CI: 35.8-43.5; $\mathrm{P}=0.003$ ).

Conclusion: ePROMs can be successfully implemented as a part of a routine multidisciplinary head and neck cancer practice. ePROMs have previously been shown to yield clinically meaningful patient-centric data to drive intervention and may improve quality of life, patient satisfaction, and overall survival. In patients with head and neck cancer, physical function varied most, with older patients and those with laryngeal primaries reporting worse physical function-related quality of life.

\section{Impact of Definitive and Timely Diagnosis on Survival of Metastatic Pancreatic Cancer in Patients With Initial Diagnosis of Cancer of Unknown Primary}

\section{Larissa White, Julie Smith-Gagen}

\section{Kaiser Permanente Colorado, Aurora, CO}

Background: Cancer of unknown primary (CUP) accounts for $3 \%-5 \%$ of all cancers and is the fourth most common cause of cancer mortality in the United States. Median survival after CUP diagnosis is 3-4 months. Pancreatic cancer (PC) accounts for $3 \%$ of all cancers and is the third most common cause of cancer mortality in the United States. Median survival after stage III/IV PC diagnosis is 2-3 months. As CUP and metastatic PC are comparable in prevalence and survival, $\mathrm{PC}$ diagnosis is a useful endpoint to assess the impact of definitive and timely diagnosis (DTD) on survival of patients initially diagnosed with CUP. We examined how DTD of metastatic PC affects survival in older patients who initially present with CUP compared to those diagnosed with metastatic PC only.

Methods: This study used 2010-2016 Surveillance, Epidemiology, and End Results (SEER)-Medicare data. Logistic regression models compared patient characteristics who received DTD in two subsets: CUP-PC and PC-only. The impact of DTD on patient survival in CUP-PC was analyzed using Cox proportional hazards regression. All models were adjusted for gender, age, race, ethnicity, area of residence, histology, and Charlson comorbidity score.

Results: The adjusted odds of DTD in CUP-PC were 15\% lower for those with a comorbidity score of 0 (adjusted odds ratio: $0.85[0.79,0.91])$ and $24 \%$ lower for epithelial/ unspecified histology (adjusted odds ratio: 0.76 [0.71, 0.82]). DTD in CUP-PC was associated with a $72 \%$ reduction in hazard of death (hazard ratio: 0.28 [0.25, 0.31]). Patients who are Black were associated with a $15 \%$ increase (hazard ratio: $1.15[1.08,1.23]$ ), Latino with a $19 \%$ increase (hazard ratio: $1.19[1.10,1.29])$, and epithelial/unspecified histology with an $85 \%$ increase (hazard ratio: 1.85 [1.76, 1.95]).

Conclusion: DTD of CUP-PC was favorable in patients who were a race other than White or Black with fewer or no comorbidities. Unfavorable characteristics included older patients and epithelial/unspecified histology. Results indicate transition in diagnosis from CUP to metastatic cancer of a known site and diagnosis within 3 months has a significant impact on the life of the patient. Our findings reflect the importance of DTD in CUP patient survival, especially for older, female, Black, Latino, and rural-residing patients with multiple comorbidities.

\section{Patient Self-Reported Experience of Serious IIIness Communication and Care Among Individuals With Advanced Cancer}

Ellis C. Dillon, Martina Li, Amandeep Mann, Amaka Agodi, Alyssa Hernandez, Hal S. Luft, Natalia Colocci, Steve Lai, Manali Patel

\section{Sutter Health, Palo Alto, CA}

Background: For individuals with advanced cancer, careful communication and early conversations regarding patients' goals, values, and preferences for care (ie, goals of care) can improve patient experiences and care at the end of life. Yet, many patients lack understanding of their diagnosis and prognosis and do not receive goal-aligned care.

Methods: A health care organization in Northern California adopted patient experience surveys as part of a quality improvement program on August 12, 2019. Individuals with advanced cancer (stage IV, recurrence with distant metastases or glioblastoma) were surveyed by mail, email, 
or phone at 1,4 , and 8 months after they were identified. Descriptive statistics and qualitative thematic analysis of open-ended questions are reported.

Results: 546 patients were identified and 403 surveys were completed at $1(n=162), 4(n=147)$, and 8 months $(n=94)$. By 1 month, 48 of 150 patients $(32.0 \%)$ reported discussing palliative care with their care team and 13 patients $(8.7 \%)$ were already receiving specialty palliative care. At 1 month, 102 of $156(65.4 \%)$ rated the quality of information received about treatment and treatment choices as "excellent" vs $59.7 \%$ at 4 months and $63.4 \%$ at 8 months. Meanwhile, 46 of $154(29.9 \%)$ at 1 month, 32 of $143(22.4 \%)$ at 4 months, and 16 of $91(17.6 \%)$ at 8 months responded "I wish I had more information about my prognosis" (vs, at 8 months, $74.7 \%$ responding "right amount of information," $0 \%$ "wished they had less information," and 7.7\% "not sure about wanting more information." Participants provided 233 responses to the open-ended question: "What could be done to improve your experience?" The most common theme was a desire for improved communication about diagnosis, prognosis, or treatment, for example, "I haven't been given how long I'll live. It would be good to have an idea." and "Discussion of what my options are. This thing happened so fast that I had radiation and didn't know what was happening."

Conclusion: For individuals with advanced cancer, these surveys suggest the biggest potential to improve experience is to improve communication, specifically discussion of treatment choices, palliative care, and prognosis. Feedback from surveys was relayed to local clinical teams, who then responded to identified concerns. Continued longitudinal surveys may reveal how patients' needs change as their illness progresses.

\section{Patients Deciding on Cancer Screening and Prevention Using Clinical Decision Support: "I Wouldn't Have Thought About Getting Screened Without It"}

Melissa Harry, Daniel Saman, Laura Freitag, Clayton Allen, Patrick J. O'Connor, JoAnn Sperl-Hillen, Joseph Bianco, Anjali Truitt, Thomas Elliott

\section{Essentia Health, Duluth, MN}

Background: Clinical decision support (CDS) designed to encourage discussions between patients and their primary care providers may help improve cancer screening and prevention rates. Our objective was to understand patients' perspectives on both cancer prevention and screening and a personalized CDS tool among those exposed to the CDS tool because they were due or overdue for cancer screening (breast, colon, lung, cervical) or prevention (human papillomavirus vaccination, tobacco, overweight).

Methods: We conducted semi-structured, qualitative interviews with 37 adult patients due or overdue for cancer prevention and screening services in 10 primary care clinics within intervention arms of a randomized control trial in a health care system serving a largely rural population. We conducted interviews immediately following a visit with a primary care provider. We thematically segmented and coded transcribed interview data using qualitative content analysis.

Results: We identified 3 overarching themes: 1) the CDS had more strengths than weaknesses, with the latter providing areas for improvement; 2) cancer prevention and screening facilitators and barriers identified were mainly at the patient level, but lack of using the CDS by some providers was the only provider-level barrier noted; and 3) discussions and decision-making varied by type of cancer prevention and screening. Almost all participants made positive comments regarding the CDS. Some participants learned new information, reported the CDS helped them make a decision they otherwise would not have made, and found the recommendations helpful in making a decision with their provider. Only 1 of the patients eligible for lung cancer screening reported speaking with their provider about it. Participants who described using the tool with their provider did have higher self-reported rates of making a choice than those who did not.

Conclusion: Participants generally approved of a personalized CDS tool for cancer screening and prevention in primary care, finding it useful and a welcome addition to their visit. However, we found some providers did not use the CDS with eligible patients, suggesting adoption issues that future research should assess, along with the impact of the CDS on screening rates. Other researchers could also apply the patient interview framework we developed in understanding patient perspectives and care needs.

\section{Identifying Individuals With Advanced Cancer: Assessing a Real-Time Alternative to Retrospective Tumor Registry Data}

Ellis C. Dillon, Amandeep Mann, Kevin Hays, Martina Li, Natalia Colocci, Manali Patel, Steve Lai, Hal S. Luft

\section{Sutter Health, Palo Alto, CA}

Background: Tumor registry data are the gold standard for cancer surveillance, but registries only report cancers at diagnosis and have long lags. Easily accessible real-time data on current cancer stage and recurrent advanced disease are needed for clinical and research initiatives. The growing use of electronic chemotherapy pathways decision support tools may help to meet this need.

Methods: ClinicalPath (formerly Via Oncology: https:// www.elsevier.com/solutions/clinicalpath) was launched at a health care organization in Northern California on February 1, 2019. This tool is designed to assist oncologists in choosing a prioritized evidence-based chemotherapy 
regimen. Oncologists input certain patient information, including stage of disease and presence of distant metastases. To understand how well data input to ClinicalPath identify patients with advanced stages of cancer, we selected all patient records in ClinicalPath consistent with advanced cancer (stage IV or distant metastases) from February 1, 2019, to September 30, 2019, to compare with organizational tumor registry (TR) data for those patients. The goal was to assess whether ClinicalPath was accurately capturing instances of advanced cancer, ie, stage IV cancer or earlier stage cancer with recurrence and distant metastases.

Results: Of 101 patients with advanced cancer identified from ClinicalPath, $85(84.2 \%)$ were found in TR data. Overall, 84 of those $85(98.8 \%)$ had the same cancer diagnosis; 56 (65.9\%) were reported in both data sources as having stage IV cancer, $20(23.5 \%)$ in the TR had an earlier stage cancer, and 9 in the TR $(10.6 \%)$ were missing staging information. One individual (1.2\%) had discrepant diagnosis (ie, TR showed prostate cancer while ClinicalPath showed non-small cell lung cancer). This patient was diagnosed with prostate cancer, which was reported in TR, and was later ( $>20$ years) diagnosed with non-small cell lung cancer, which was noted in ClinicalPath. There were 16 patients not in TR data, likely because they came to the organization after being diagnosed elsewhere.

Conclusion: Information in ClinicalPath is consistent with the TR for the majority of patients with advanced cancer and captures patients whose TR data is submitted from other organizations. Being embedded in the clinical workflow, ClinicalPath offers reliable and faster evidence-based data for research and clinical quality improvement efforts.

\section{Improving Access to Specialty Radiotherapy for Patients With Resectable Brain Tumors}

Mehee Choi, Brian Martin, Joseph Zabramski, Lisa Misell, David Brachman

\section{GT Medical Technologies, Tempe, AZ}

Background: Many patients with brain tumors face challenges with access to care. For rural patients, prolonged travel times may limit access to appropriate radiotherapy. Radiation centers offering specialized brain radiotherapy, eg, stereotactic radiosurgery (SRS), are geographically limited. Utilization of brain brachytherapy, localized radiation undertaken at the time and place of resection, could help lessen this access-to-care disparity. Until recently, technical challenges have limited the adoption of brachytherapy, but the U.S. Food and Drug Association recently cleared an easier-to-use permanently implanted, bioresorbable collagen square embedded with Cesium-131 sources (GammaTile, GT Medical Technologies) for implantation at surgery for all recurrent intracranial tumors and for newly diagnosed malignant intracranial neoplasms. To investigate if wider availability of this treatment could possibly lower the geographic barrier to access to care, we mapped the U.S. population against existing radiation centers with brain tumor treatment expertise and neurosurgery centers performing craniotomies.

Methods: We analyzed 2018 Centers for Medicare \& Medicaid Services claims data using CPT codes for singleand multifraction SRS to identify radiation centers with brain tumor treatment expertise and mapped these against the population. Using similar methodology, using CPT codes for craniotomies, we identified neurosurgery centers, as any facility performing craniotomies is potentially eligible to implant GammaTile.

Results: A total of 135 radiation centers used CPT codes for SRS; 193 million, 119 million, 82 million, and 52 million Americans lived $>30,>60,>90$, and $>120$ minutes from one of these centers, respectively. A total of 530 neurosurgery centers perform craniotomies, including 1 or more in every state, a 4-fold increase over the number of radiation centers offering SRS.

Conclusion: For many patients, substantial travel distances limit their access to radiation centers with brain tumor treatment expertise. In contrast, the 530 craniotomyperforming neurosurgery centers have far greater geographic dispersion. The option of undergoing brain radiation with GammaTile implantation at the time of brain tumor craniotomy brings treatment closer to millions, ensures compliance, and reduces additional travel for follow-up radiation treatment.

\section{Diffusion of Newly Recommended Annual Colorectal Cancer Screening Among African Americans 45-49 Years Old}

Gloria Coronado, Andrea N. Burnett-Hartman, Jeff Lee, Carmit McMullen, Carolyn Rutter, Caitlin Murphy, Mary Ann McBurnie, John M. Caruthers, Amanda Petrik, Samantha Hendren

\section{Kaiser Permanente Northwest, Portland, OR}

Background: Over the past two decades, there has been an unexplained rise in colorectal cancer incidence in individuals younger than age 50. In response to this rise, in 2020, the U.S. Preventive Services Task Force (USPSTF) updated its colorectal cancer screening recommendations; the new draft recommendations call for screening initiation among average-risk individuals to begin at age 45, instead of 50 . Since 2016, Kaiser Permanente Northwest has recommended colorectal cancer screening for African Americans who are 45-49 years old, and these patients are included in an annual centralized mailed fecal immunochemical test (FIT) outreach program. Little is known about the uptake of colorectal cancer screening in this population subgroup. This information can provide important clues about how 
quickly screening recommendations are implemented in delivery systems.

Methods: Patients were 45-49-year-old African Americans members of Kaiser Permanente Northwest identified from 2016 through 2020. We report the proportion of eligible patients who completed a FIT each year and the proportion that completed 1, 2, 3, and 4 FITs over the 4-year evaluation interval.

Results: Our study sample included 923 African Americans in the 45-49 age range; $41 \%$ of the sample completed at least one FIT over the 4-year interval. This included $22.3 \%$ who screened only once, $11.9 \%$ who screened twice, $5.7 \%$ who screened 3 times, and less than $2 \%$ who screened each of the 4 years.

Conclusion: Despite the annual promotion of FIT among African Americans 45-49 years of age, only 41\% completed at least one FIT over the 4-year interval. Additional efforts are needed to successfully promote colorectal cancer screening initiation in this group. Given the pending changes to USPSTF guidelines to drop the age of screening initiation to 45 years, our findings may inform the diffusion of colorectal cancer screening in the context of new screening guidelines.

\section{CARDIOVASCULAR DISEASE}

\section{Comparing Change in Systolic Blood Pressure With Clinic-Based Care Versus Telehealth Care in a Pragmatic Cluster-Randomized Trial (Hyperlink 3)}

Karen L. Margolis, JoAnn Sperl-Hillen, A. Lauren Crain, Jeffrey Anderson, Anna Bergdall, Leif I. Solberg, MarySue Beran, Beverly B. Green, Pamala A. Pawloski, Jeanette Y. Ziegenfuss, Amy Kodet, Daniel Rehrauer, Meghan JaKa, Christine Norton, Patricia Haugen, Benjamin Crabt, Nicole Trower, Deepika Appana, Rashmi Sharma, Sarah Norman, Patrick J. O'Connor

\section{HealthPartners, Minneapolis, MN}

Background: Uncontrolled hypertension is the largest contributor to all-cause and cardiovascular mortality. Nurseand pharmacist-led telehealth care (TC) interventions have resulted in large, lasting improvements in blood pressure compared with clinic-based care (CC) under controlled research conditions but have not been studied at scale under real-world conditions.

Methods: This cluster-randomized trial in 21 primary care clinics used electronic health record algorithms triggered during primary care encounters to enroll patients 18-85 years old with hypertension and blood pressure of $>150 / 95$ mmHg. TC patients $(n=1424)$ were recommended to follow up with a pharmacist in 2 weeks to obtain home blood pressure telemonitoring and telephone management visits. CC patients $(n=1648)$ were recommended to follow up with a medical assistant for a blood pressure check in 2 weeks. The primary outcome was the difference in change in systolic blood pressure (SBP) between groups over 12 months using blood pressure data from the electronic health record. A secondary per protocol analysis among patients who enrolled and adhered to recommended follow-up within 6 weeks used inverse probability weighting to account for potential bias.

Results: Enrolled patients had a mean age of 60 years and were 47\% male, 7\% Asian, 19\% Black, 69\% White, and $2 \%$ Hispanic. Mean enrollment blood pressure was 164/94 mmHg. Within 6 weeks, $27 \%$ of TC patients had a pharmacist visit (of whom $80 \%$ began telemonitoring) and 32\% of CC patients had a medical assistant visit. Change in SBP was similar in TC (-18.7 $\mathrm{mmHg})$ and $\mathrm{CC}(-17.5 \mathrm{mmHg})$ patients (difference of $-1.2 \mathrm{mmHg}$ [95\% CI: -2.9, 0.6]). However, in the per protocol analysis, TC patients had greater decrease in SBP than CC patients $(-4.6 \mathrm{mmHg}[95 \% \mathrm{CI}:-7.7,-1.5])$. Patient surveys showed higher ratings of care, more frequent self-monitoring, and greater use of home blood pressure for treatment changes in TC.

Conclusion: Patient engagement in the TC intervention was much lower in this pragmatic trial than in a previous explanatory trial, and the primary analysis showed equivalent decrease in SBP in both groups. However, among those who engaged in follow-up care, TC patients had a larger decrease in SBP. Additional research is needed to study effects of strategies to encourage uptake of telehealth care in realworld patients with hypertension.

\section{Blood Pressure Checks and Diagnosing Hypertension: Provider Knowledge, Practices, and Beliefs}

Beverly B. Green, Melissa Anderson, Andrea Cook, Kelly Ehrlich, Yoshio Hall, Clarissa Hsu, Dwayne Joseph, Karen L. Margolis, Jennifer McClure, Sean Munson, Matthew Thompson

\section{Kaiser Permanente Washington Health Research Institute, Seattle, WA}

Background: The U.S. Preventive Services Task Force recommends out-of-office blood pressure (BP) measurement, using 24-hour ambulatory (ABPM) or home BP monitoring, before making a new diagnosis of hypertension. However, this approach is not common in routine practice. This study's objective was to evaluate provider knowledge, beliefs, and practices about BP diagnostic tests.

Methods: The setting was 10 primary care medical centers in a single health care system. Emails with a link to a survey were sent to 420 providers, with $282(67 \%)$ responding, including 102 medical assistants (MA), 28 licensed practical nurses (LPN), 33 registered nurses (RN), 86 primary care physicians (physician), and 33 advanced practitioners 
(AP). Outcomes were a descriptive analysis of provider BP measurement and hypertension-diagnosis knowledge, beliefs, and practices.

Results: Most providers (78.8\%) believed that BP measured manually with a stethoscope and ABPM was a very or highly accurate way to measure BP when making a new diagnosis of hypertension. In contrast, most providers did not believe that automated clinic BP, home BP, or kiosk $\mathrm{BP}$ measurements were very or highly accurate. Almost all providers reported that they always or almost always relied on clinic BP measurements in making a new diagnosis of hypertension (95.7\%), but the majority of physicians/ APs would prefer using ABPM (60.5\%) if it was readily available. Providers were mixed as to whether patients received home BP training and whether home BP monitors were checked for accuracy. Physicians/APs reported varying home BP schedules, with no schedule the most common response $(37.3 \%)$, followed by 14 days (19.1\%) and 7 days (10.9\%). Almost all physicians/APs reported using a clinic BP threshold of 140/90 $\mathrm{mmHg}$ for making a new diagnosis, but a few reported $130 / 80 \mathrm{mmHg}$. Very few physicians/APs reported guideline-concordant home or ABPM diagnostic thresholds, with $140 / 90 \mathrm{mmHg}$ the most common response.

Conclusion: In our study, providers did not report following evidence-based guidelines for making a new diagnosis of hypertension and appeared to have substantial gaps in knowledge, beliefs, and use of recommended practices. Interventions to increase use of evidence-based practices for diagnosing hypertension are needed.

\section{Blood Pressure Checks and Diagnosing Hypertension (BP-CHECK): A Randomized Controlled Trial}

Beverly B. Green, Melissa Anderson, Andrea Cook, Kelly Ehrlich, Yoshio Hall, Clarissa Hsu, Dwayne Joseph, Predrag Klasnja, Karen L. Margolis, Jennifer McClure, Sean Munson, Matthew Thompson

\section{Kaiser Permanente Washington Health Research Institute, Seattle, WA}

Background: The U.S. Preventive Services Task Force recommends out-of-office blood pressure (BP) measurement, using 24-hour ambulatory (ABPM) or home BP monitoring, before making a new diagnosis of hypertension. However, this approach is not common in routine practice. The objective of this randomized controlled trial was to compare the performance and acceptability of clinic, home, and kiosk-based BP monitoring to ABPM for making a new diagnosis of hypertension.

Methods: The setting was 12 Kaiser Permanente Washington primary care medical centers. The population was 510 adults 18-84 years old without diagnosed hypertension and on no antihypertensive medications who registered elevated BP in clinic and at a baseline research visit. Participants were randomized to 1 of 3 regimens for diagnosing hypertension - 1) Clinic BP (at follow-up clinic appointment), 2) Home BP (duplicate BP measurements twice a day for 5 days), or 3) Kiosk BP (triplicate BP measurements over 3 days) - at any time over 3 weeks. All participants subsequently completed ABPM. All BP data were collected electronically. The primary outcome was the diagnostic performance of each method as compared to ABPM. Secondary outcomes include adherence to the assigned protocol and patientreported outcomes. Linear regression models were used to estimate the mean difference in systolic BP and diastolic BP between the diagnostic and ABPM reference standard measures for each group.

Results: Overall, 78.8\% (402 of 510) of the participants completed the prespecified minimum number of $\mathrm{BP}$ measurements needed for diagnostic assessments. Clinic, Home, and Kiosk BP monitoring adherence was $87.2 \%$, $90.6 \%$, and $67.9 \%$, respectively, with $91.6 \%$ completing ABPM. Compared to mean daytime ABPM, difference in adjusted mean systolic BP for Clinic was $-4.7 \mathrm{mmHg}(95 \%$ CI: $-7.2-2.1 ; \mathrm{P}<0.001)$, for Home was $-0.1 \mathrm{mmHg}(95 \% \mathrm{CI}$ : $-1.6,1.5 ; \mathrm{P}=0.92)$, and for Kiosk was $9.0 \mathrm{mmHg}(95 \% \mathrm{CI}$ : 7.0, 11.1; $\mathrm{P}<0.001)$. Difference in adjusted mean diastolic BP for Clinic was $-6.7 \mathrm{mmHg}$ (95\% CI: $-8.2,-5.1 ; \mathrm{P}<0.001)$, for Home was $-0.3 \mathrm{mmHg}(95 \% \mathrm{CI}:-1.3,0.8 ; \mathrm{P}=0.81$ ), and for Kiosk was $3.9 \mathrm{mmHg}$ (95\% CI: 2.6, 5.3; $\mathrm{P}<0.001$ ). Sensitivity of Clinic, Home, and Kiosk for detecting hypertension was $31.1 \%, 81.7 \%$, and $95.1 \%$, and specificity was $79.5 \%, 53.2 \%$, and $26.7 \%$, respectively. Home BP was preferred. ABPM was more likely to disturb sleep and be uncomfortable, but participants perceived it as accurate.

Conclusion: Home BP had better diagnostic performance, adherence, and acceptability than clinic or kiosk.

\section{Telehealth Versus Self-Directed Lifestyle Intervention to Promote Healthy Blood Pressure: Study Design and Rationale}

Alex Chang, Christina Yule, Sara Kwiecien, Charlotte Collins, Allison Naylor, Stephen Juraschek, Lisa Bailey-Davis

\section{Geisinger Research, Danville, PA}

Background: Weight loss, consumption of a Dietary Approaches to Stop Hypertension (DASH) dietary pattern, reduced sodium intake, and increased physical activity have been shown to lower blood pressure (BP). Use of webbased tools and telehealth to deliver lifestyle counseling could yield potentially scalable solutions to improve blood pressure through behavioral modification, though limited data exist to support these approaches in clinical practice. Methods: This randomized controlled trial is designed to 
compare the efficacy of a telehealth vs self-directed lifestyle intervention in lowering 24-hour systolic BP in patients with overweight/obesity (body mass index of $\geq 25 \mathrm{~kg} / \mathrm{m}^{2}$ ) and 24hour systolic BP of $120-160 \mathrm{mmHg}$. All participants are to receive personalized recommendations to improve dietary quality based on a web-based food frequency questionnaire, access to an online comprehensive weight management program, and a smartphone dietary app. The telehealth arm additionally includes weekly calls with registered dietitian nutritionists who use motivational interviewing. The primary outcome is change from baseline to 12 weeks in 24-hour systolic BP. Secondary outcomes include changes from baseline in 24-hour diastolic BP, daytime systolic $\mathrm{BP}$, nighttime systolic BP, daytime diastolic BP, nighttime diastolic BP, total Healthy Eating Index-2015 (HEI) score, weight, waist circumference, and physical activity. Other prespecified outcomes include change in individual components of the HEI score and satisfaction with the Healthy BP research study, measured on a 5-point Likert scale. Exploratory analyses are designed to test whether outcomes differed before and after the pandemic.

Results: Out of 210 participants who were consented for the first study visit, 169 have completed the run-in period and have been randomized into the trial. Recruitment and trial enrollment continued during the pandemic with conversion to remote study visits. In a preplanned interim analysis of the first 34 randomized patients, 28 (82\%) completed the 3-month study visit. Mean (standard deviation) changes from baseline to 3 months were $-8.0 \mathrm{mmHg}$ (9.9) for 24hour systolic BP, $-5.4 \mathrm{~kg}$ (4.5) for weight, $-6.1 \mathrm{~cm} \mathrm{(7.4)} \mathrm{for}$ waist circumference, and 11.2 units (12.0) for HEI score.

Conclusion: Completion of the study is expected by summer of 2021 and will determine the comparative effectiveness of a telehealth vs self-directed approach to lower 24-hour systolic BP using web-based tools.

\section{Provider Knowledge and Practice Patterns Regarding the Measurement and Management of Hypertension}

Ahana Bhan, Kathryn Foti, Jamie Green, Cybele Pacheco, Ion Dan Bucaloiu, Alex Chang

\section{Geisinger Research, Danville, PA}

Background: Studies show that blood pressure (BP) control has worsened in recent years despite 2017 American College of Cardiology (ACC)/American Heart Association (AHA) guidelines recommending lower BP targets $(<130 / 80$ $\mathrm{mmHg}$ ) for many adults with hypertension. Little data exist on provider knowledge and consistency of practice patterns with ACC/AHA guidelines.

Methods: A 31-item hypertension survey was emailed in January 2021 to providers involved in hypertension care at Geisinger, including those practicing in family medicine/internal medicine, nephrology, cardiology, and endocrinology. The survey consisted of 31 questions about provider characteristics, knowledge of BP measurement, use of out-of-office BP measurement, and practice patterns for hypertension management. Practice patterns were assessed based on responses to questions about BP goals in various clinical scenarios (eg, younger/older, no comorbidity/ various comorbidities, good/poor functional status).

Results: Out of a total of 584 health care providers, 173 providers (35\% attending physicians, 28\% advanced practitioners, $20 \%$ pharmacists, $18 \%$ residents/fellows) completed the survey, a $29.6 \%$ response rate. The majority $(53 \%)$ of providers reported repeating office BP measurements $75 \%-100 \%$ of the time if BP was above their target goal. Nearly all (99\%) providers reported using home $\mathrm{BP}$ monitoring in patients with uncontrolled BP; the most common way of receiving BP results was having the patient bring results to clinic (52\%). Only 42\% correctly answered that mineralocorticoid receptor antagonists were the drug of choice for uncontrolled BP after maximum doses of angiotensin-converting enzyme inhibitors, dihydropyridine calcium channel blockers, and thiazide diuretic. In most patient scenarios for which treatment to $<130 / 80 \mathrm{mmHg}$ is recommended by the AHA, the majority of providers did not report typically treating to that level. For example, providers reported treating a hypothetical 85-year-old healthy patient with good functional status less aggressively (47\% to $<150 / 90,45 \%$ to $<140 / 90$, and $8 \%$ to $<130 / 80 \mathrm{mmHg}$ ) than an 85 -year-old patient with diabetes $(8 \%$ to $<150 / 90,65 \%$ to $<140 / 90$, and $26 \%$ to $<130 / 80 \mathrm{mmHg}$ ). Family medicine providers were more likely to choose higher BP targets.

Conclusion: Health care providers' clinical practices play a crucial role in achieving adequate BP control in the population. Additional work is needed to enhance capture of home BP measurements and address challenges to implementing lower BP goals.

\section{Blood Pressure Control and Practice Patterns During the COVID-19 Pandemic}

Alex Chang, Ahana Bhan, Kathryn Foti, Jamie Green, Cybele Pacheco, Ion Dan Bucaloiu

\section{Geisinger Research, Danville, PA}

Background: Recent data suggest worsening blood pressure (BP) control in U.S. adults with hypertension. The impact of the COVID-19 pandemic on BP control has not been well studied.

Methods: We used electronic health record (EHR) data to examine trends in BP control and adherence to hypertension guidelines among adults 18 years of age or older with hypertension diagnosis codes who received care at Geisinger from January 1, 2018, to October 12, 2020. BP control of $<140 / 90 \mathrm{mmHg}$ was defined by averaging all outpatient BP values in the EHR over each calendar year for each patient. 
Trends in BP control were examined overall and stratified by the prior year's mean BP as $\geq 140 / 90,<140 / 90$, or unavailable.

Results: There were 127,362, 132,780, and 109,589 patients with hypertension and outpatient BP measurements in 2018, 2019, and 2020, respectively. Patient characteristics were similar for each calendar year (mean age of 62.9 years, 50.8\% female, $35.6 \%$ with diabetes, $29.1 \%$ with chronic kidney disease, $5.5 \%$ with myocardial infarction, and $12.5 \%$ with heart failure). Prevalence of uncontrolled BP of $>140 / 90 \mathrm{mmHg}$ was $25.1 \%$ in $2018,24.9 \%$ in 2019 , and worsened to $27.2 \%(\mathrm{P}<0.001)$ in 2020 . The prevalence of uncontrolled BP was higher in 2020 compared to earlier years regardless of the prior year's mean BP classification (prior BP of $\geq 140 / 90$ : $48.2 \%$ remained uncontrolled in 2019 vs $50.7 \%$ in 2020; prior BP of $<140 / 90$ : $13.9 \%$ became uncontrolled in 2019 vs $15.2 \%$ uncontrolled in 2020; no BP in prior year: $37.5 \%$ were uncontrolled in 2019 vs 39.6\% uncontrolled in 2020). Among patients with hypertension seen during the study period, $74.5 \%$ were on first-line antihypertensive drugs (renin-angiotensinaldosterone system inhibitor: $61.9 \%$; thiazides: $27.7 \%$; calcium channel blockers: $25.0 \%$ ), while $34.2 \%$ were on beta blockers; the mean number of antihypertensive classes was 1.83 (standard deviation: 1.3). In 2020, 12.5\% of visits were conducted by telehealth, and $<1 \%$ of these telehealth visits had BP values recorded in structured EHR fields.

Conclusion: BP control worsened slightly in a large health system during the COVID-19 pandemic. Further investigation is needed to understand how unknown BP measurements may have impacted hypertension care. Opportunities to improve BP control include enhancing capture of home BP measurements to enable appropriate titration and addition of BP medication classes.

\section{The Impact of the COVID-19 Pandemic on Cardiovascular Hospitalizations}

\section{Suma Vupputuri, Kevin B. Rubenstein, Celeena R. Jefferson, Sadiya S. Khan}

\section{Kaiser Permanente Mid-Atlantic States, Rockville, MD}

Background: During the early phases of the COVID-19 pandemic, significant declines in hospitalizations for cardiovascular disease (CVD) were observed. Potential explanations for this phenomenon include health systemlevel factors (eg, limiting elective procedures) as well as individual-level factors (eg, avoidance of health care due to fear). These may have led to delays in care, and it is not known if a "rebound" effect resulting in greater than normal CVD hospitalizations may have occurred. Therefore, we aimed to examine rates of CVD hospitalization following the onset of the COVID-19 pandemic compared to rates of CVD hospitalization prior to the pandemic.
Methods: We conducted a retrospective, serial crosssectional study among adults ( $\geq 18$ years old) with CVD hospitalization during two periods: 1) following the onset of the COVID-19 pandemic (March 1, 2020, to November 30, 2020); and 2) the prepandemic period (March 1, 2019, to November 30, 2019). CVD events were defined by emergency department or inpatient primary diagnoses for ischemic heart disease, stroke, and peripheral vascular disease. Rates of CVD hospitalization were reported for 3-month intervals within the pandemic and prepandemic periods per 100 members.

Results: During the COVID-19 and prepandemic periods, 2503 and 2864 patients were hospitalized for CVD, respectively. Patients hospitalized during the pandemic had a mean (standard deviation) age of 67 (14) years, were predominantly Black $(45 \%)$ and White $(35 \%)$, and the majority were male (58\%). There were no differences in demographic characteristics between patients hospitalized for CVD in the COVID-19 pandemic and prepandemic periods. Rates of CVD hospitalization were lower during the COVID-19 period (12.1, 13.1, and 14.3 per 100 members for time intervals with midpoints at April 15, 2020, July 15, 2020, and October 15, 2020, respectively) than the prepandemic period $(15.4,16.1,14.3$ per 100 members for time intervals with midpoints at April 15, 2019, July 15, 2019, and October 15, 2019, respectively).

Conclusion: Rates of CVD hospitalization were significantly lower following the onset of the COVID-19 pandemic and did not normalize until late in 2020. No rebound effect of increased CVD hospitalization was noted during our study period. Future efforts should investigate whether the severity of outcomes due to CVD hospitalization differ during the pandemic relative to the prepandemic period, following potential delays in health care utilization.

\section{CHRONIC CONDITIONS, MULTIMORBIDITY, AND AGING POPULATIONS}

\section{Association Between Spiritual Distress With Symptoms and Quality of Life in Patients Receiving Home-Based Palliative Care}

Ellie Fratt, Andre Cipta, Bethany Turner, Eric Haupt, Emily Rozema, Huong Nguyen

Kaiser Permanente Southern California, Pasadena, CA

Background: Spiritual distress may exacerbate other symptoms and negatively affect quality of life. The purpose of this analysis is to determine the association between spiritual distress with symptoms and quality of life in patients receiving home-based palliative care (HBPC). 
Methods: Data for this analysis were drawn from a comparative effectiveness trial of two HBPC models (standard vs tech-supported) for patients with serious illness and their caregivers. Research staff contacted patients and caregivers via phone within 14 days from the patient's admission to HBPC. Survey responses on the Edmonton Symptom Assessment Scale (ESAS) and PROMIS-10 tool for measuring quality of life were either obtained directly from the patient or via the caregiver (assisted or proxy report). Spiritual distress was assessed with a validated question using the following anchors: 0 $=$ no spiritual pain/at peace to $10=$ worst spiritual pain/ not at peace; patients were categorized as having no (0), mild (1-5), or moderate/severe (6-10) spiritual distress. Sociodemographic characteristics were extracted from the patient's electronic medical record.

Results: The sample included 658 patients (mean age: $78 \pm 13$ years; $60 \%$ female; $50 \%$ White). The Charlson comorbidity index was $8.3 \pm 3.3$. Over one-quarter of the sample $(n=181)$ had moderate to severe spiritual distress, and $48 \%$ endorsed some level of spiritual distress upon admission to HBPC. There were no differences in sociodemographics or religious affiliation across spiritual distress groups except for race/ ethnicity. Patients who were identified as belonging to Hispanic, Black/African American, or Other racial/ethnic groups more often reported moderate to severe spiritual distress than non-Hispanic Whites (32\% vs $32 \%$ vs $43 \%$ vs $21 \%$, respectively; $\mathrm{P}=0.02$ ). Symptom burden per ESAS $(29.8 \pm 14.9$ vs $35.5 \pm 15.0$ vs $42.5 \pm 14.5)$ and qualify of life per PROMIS-10 (physical: $32.3 \pm 7.1$ vs $30.6 \pm 5.9$ vs 28.6 \pm 6.0 ; mental: $41.2 \pm 9.1$ vs $38.4 \pm 8.3$ vs $34.8 \pm 7.3$ ) were significantly worse with increasing spiritual distress from none, mild, and moderate/severe, respectively $(\mathrm{P}<0.001$ for all). Results of the adjusted analyses will be presented at the meeting.

Conclusion: Spiritual distress is correlated with significantly worse symptoms and quality of life. Prevalence of moderate to severe spiritual distress varies by race/ethnicity. It is important to assess for and address spiritual distress when optimizing symptom management and quality of life, particularly within vulnerable sociodemographic groups.

\section{Time-Series Analysis of Recent Antidiabetic Medication Prescribing Trends Among Medicare Enrollees in an Integrated Health System}

Michael Miller, Anna DiNucci, Rabia Jalalzai, Nonna
Rabinovich, Douglas Roblin

Kaiser Permanente Mid-Atlantic States, Rockville, MD

Background: As prevalence of type 2 diabetes mellitus (T2DM) has grown, numerous therapeutic options have emerged with new evidence and revised treatment guidelines. Despite high T2DM prevalence in Medicare enrollees $(31.6 \%$ in 2015), recent U.S. prescribing trends (post-2015) for antidiabetic medications remain unreported. Therefore, this research describes recent monthly prescribing trends for antidiabetic medications in a large, diverse population of Medicare enrollees from Mid-Atlantic states.

Methods: Demographic, diagnostic, clinical encounter, and prescribing data between July 2018 and July 2020 for Medicare enrollees 65 years of age or older with a prescription drug benefit and T2DM were extracted from the electronic health record in a large integrated health system. The total number of unique prescription orders for antidiabetic medications was summed and reported as the monthly prescribing rate per 100 eligible plan members. Cross-sectional time-series regression models with NeweyWest standard errors and up to a 6-lag order were estimated for the 25-month observation period to describe the monthly prescribing trends overall and by pharmaceutical subclasses: first-generation (biguanides, thiazolidinediones, sulfonylureas, alpha-glucosidase inhibitors, dopamine receptor agonists, meglitinide analogs), second-generation (glucagon-like peptide-1 agonists, sodium glucose cotransporter-2 inhibitors, dipeptidyl peptidase-4 inhibitors), human insulins, insulin analogs, and combination medications. Separate models were constructed for all monthly-eligible cross-sectional samples and continuouslyeligible samples.

Results: For all monthly-eligible members $(n>22,000)$, the time-series model estimated the overall baseline month prescribing rate as $\beta<$ sub $>0</$ sub $\geq 23.88 \mathrm{~T} 2 \mathrm{DM}$ medication orders per 100 members, with a significant positive monthly trend $(\beta<$ sub $>1</$ sub $\geq 0.12) ; \mathrm{P}<0.05$. At baseline, first-generation antidiabetic medication orders were most common $(\beta<$ sub $>0</$ sub $\geq 16.28$ medication orders per 100 eligible members), followed by human insulins $(\beta<\operatorname{sub}>0</$ sub $\geq 3.87)$, insulin analogs $(\beta<\operatorname{sub}>$ $0</$ sub $\geq 3.04$ ), second-generation antidiabetic medications ( $\beta<$ sub $>0</$ sub $\geq 0.58$ ), and combination medications ( $\beta$ $<\operatorname{sub}>0</$ sub $\geq 0.11)$. Significant positive monthly trends were observed for human insulins, insulin analogs, and second-generation pharmaceutical subclasses $(\mathrm{P}<0.05)$. For continuously-eligible members $(n=19,185)$, no significant monthly trend for overall antidiabetic medication orders was observed. However, similar significant positive monthly prescribing trends were observed for human insulins, insulin analogs, and second-generation pharmaceutical subclasses $(\mathrm{P}<0.05)$.

Conclusion: Increasing monthly T2DM antidiabetic medication prescribing trends were observed for some pharmaceutical subclasses. Future work will focus on patient-level impact with respect to T2DM management and subsequent treatment outcomes. 


\section{Experiences of Caregivers of Persons Living With Dementia Receiving Home-Based Primary Care}

Jessica D. Vallejo, Mayra Macias

Kaiser Permanente Southern California, Pasadena, CA

Background: Home-based primary care (HBPC) services are essential to the care of homebound persons living with advanced dementia (PLWD). We sought to describe the experience of family caregivers receiving HBPC services within an integrated health system to identify improvement opportunities.

Methods: Using email and phone outreach, we recruited family caregivers of PLWD who were actively receiving care $(n=129)$ from October 6, 2020, to November 20, 2020, oversampling underrepresented groups. Semi-structured interview questions probed the following domains prioritized by the program leaders: satisfaction with HBPC services, areas for improvement or unmet needs, and experience with virtual care during the pandemic. Interviews were conducted in English or Spanish and were audio-recorded and transcribed. Transcripts were imported to NVivo where an iterative process, informed by grounded theory, was used to identify emergent themes.

Results: A total of 16 caregivers ( $81 \%$ female, $63 \%$ Hispanic, $19 \%$ spoke Spanish) were interviewed. Most caregivers were 50 years or older $(69 \%)$ and adult children or other family members (88\%); approximately one-third reported receiving minimal to no caregiving help from others (32\%). Caregivers reported that HBPC met $(31 \%)$ or exceeded $(56 \%)$ their expectations. The interviews highlighted 4 important themes: caregivers' appreciation for the coordinated, continuous, convenient, and affordable care provided by HBPC; informational and emotional support for the caregiver; desire for additional assistance with personal care needs and costs of medical supplies and equipment; and areas for improvement related to better communications around program expectations, after-hours support, and quality of care with contracted home health staff. Caregivers felt there should be a balance between virtual care and necessary in-person visits due to the complexity of patient needs and technology challenges.

Conclusion: Similar to previous reports, we also found that for most family caregivers of PLWD, HBPC was effective in providing coordinated, continuous, and convenient care aligned with families' priorities. However, caregivers also expressed unmet service and financial needs and desired improved communications and quality oversight for other contract services. Expansion of HBPC services will be contingent on not only improving care experience for patients and families but also more effective resource utilization for health systems and payers.
IMPACT Learning Health Network: Transforming Care Through Embedded Pragmatic Clinical Trials in Health Care Settings That Serve People With Dementia

Leah Tuzzio, Leah Hanson, Ula Hwang, Rosa Baier, Rachel Hays, Eric Larson

Kaiser Permanente Washington, Seattle, WA

Background: More than 5 million Americans are living with Alzheimer's disease or a related dementia, which is expected to triple in the next 30 years. People living with dementia (PLWD) have higher rates of hospital stays, emergency room visits, skilled nursing facility stays, and overall health care utilization. Care partners experience increased anxiety, depression, and stress. The disconnect between health care settings and evidence-based care for PLWD has stymied the real-world health care setting from adoption of promising interventions. Care settings that embrace a culture of continuous learning are ideal settings for embedded pragmatic clinical trials (ePCTs), which can accelerate evidence generation in a cycle that creates sustainable improvements in clinical practice.

Methods: The mission of the NIA IMPACT Collaboratory is to build the nation's capacity to conduct ePCTs of nonpharmacologic interventions embedded within health systems for PLWD and their care partners. Over the past year, IMPACT developed a learning health network (LHN), a consortium of individuals representing care settings and organizations joined by the common goal of improving care quality, equity, and accessibility for PLWD and their care partners. The LHN is intended to comprise 4 communities, bringing together stakeholders and catalyzing collaboration to build capacity for ePCT research: 1) longterm care (nursing homes, assisted living facilities); 2) health care settings (ambulatory care clinics, Accountable Care Organizations, Medicare Advantage plans, integrated delivery systems); 3) hospitals/emergency departments (inpatient, hospital at home, prehospital); and 4) communitybased organizations (Meals on Wheels, hospice, senior centers, adult day centers).

Results: Thus far, 3 communities have formed within the LHN. Topics discussed have included setting-specific priorities for dementia care, common interests in dementia research, challenges within each setting to implement ePCTs with this population, and how to collaborate. Currently, there are multiple IMPACT funding opportunities to support research collaboration in this area.

Conclusion: This LHN is building a national resource devoted to the intersections between ePCTs for persons with dementia and the setting in which they receive health care. The LHN seeks to engage investigators and sites within the HCSRN and other health care settings to establish a collaborative to support and facilitate the conduct of ePCTs among PLWD and their care partners. 


\section{Multimorbidity Among Nursing Home Residents With Obesity}

Ning Zhang, Yanhua Zhang, Junkai Xie, Chongli Yuan, Danni Liu, John Zeber, Arlene Ash, Min Zhang

\section{Meyers Primary Care Institute, Worcester, MA}

Background: A steady increase has occurred in the prevalence of obesity among nursing home residents, from $22 \%$ in 2005 to $28 \%$ in 2015 ; meanwhile, the prevalences of other weight categories have all decreased. Obesity develops frequently in the presence of other chronic conditions or cognitive impairment. Thus, nursing home care providers must manage obesity, comorbidities, and functional limitations. The objectives of this project are to characterize constellations of multimorbidity among nursing home residents with obesity.

Methods: This study is a secondary data analysis based on the Minimum Data Set in 2014. The study sample includes residents with a body mass index (BMI, defined as weight in kilograms divided by height in meters squared) of $\geq 30$. Residents were categorized as having class I $(30 \leq \mathrm{BMI}<$ $35)$, class II $(35 \leq \mathrm{BMI}<40)$, or class III (BMI $\geq 40)$ obesity. Multimorbidity represents if a resident had any chronic conditions, functional limitations, and geriatric conditions (ie, conditions commonly experienced by older adults that are not included in the chronic disease paradigm). Other variables of interest included age, sex, race, and ethnicity. We used association rule mining to identify the most common monads, dyads, triads, quadriads, and pentads. In addition, we identified the association between the cooccurring conditions.

Results: Among nursing home residents with obesity, the most common condition was hypertension (75\%) presenting with or without the co-occurrence of any other conditions. The most common dyad was hypertension and urinary incontinence (52\%). The most common triads were hypertension, urinary incontinence, and bowel incontinence $(37 \%)$. The most common quadriads were hypertension, urinary incontinence, bowel incontinence, and moderate to severe cognitive impairment $(23 \%)$. The most common pennads were hypertension, urinary incontinence, bowel incontinence, moderate to severe cognitive impairment, and depression (37\%). Class III obesity has a slightly different combination of multimorbidity than the other two groups. Bowel incontinence and urine incontinence have a strong correlation.

Conclusion: Nursing home residents with obesity frequently have hypertension, depression, moderate physical dependence, and some geriatric conditions. Clinical practice guidelines should address how these conditions in combination impact care processes and contribute to undesirable outcomes such as hospitalization and mortality.

\section{Examining Long-Term Weight Loss Surgery Success in a National Electronic Health Record Dataset}

Megan Ferriby Ferber, Timothy Chrusciel, Joanne Salas, Jeffrey Scherrer

\section{Saint Louis University / SSM Health, St. Louis, MO}

Background: As rates of obesity continue to rise, so do the number of patients seeking weight loss surgery (WLS). This study utilized a private sector electronic health record (EHR) database to determine if patients' sex, racial identity, and presurgical comorbidities were associated with postWLS success (ie, losing $\geq 50 \%$ excess weight) out to 4 years postsurgery.

Methods: We used a national EHR database of 5 million adults. Our study cohort was created using ICD-9 and ICD10 codes to identify patients who had WLS in 2012 to 2015. Additional inclusion criteria were baseline body mass index (BMI) of 35 or greater and a valid BMI for at least 1 followup period. Successful weight loss was defined as the loss of at least $50 \%$ of the weight needed to reach a BMI of 25 . Patient characteristics were compared at 4 follow-up periods: $1,2,3$, and 4 years postsurgery. Data were analyzed using chi-squared tests for categorical variables and independent $t$-tests for continuous variables.

Results: Results indicated $66.2 \%$ of patients experienced successful weight loss at 1 year post-WLS. Significant differences $(\mathrm{P}<0.05)$ were associated with female sex, White race, younger age, lower baseline BMI, and 8 of the 15 comorbidities examined. At 2 years post-WLS, 59.4\% of patients experienced successful weight loss, with White race, younger age, lower baseline BMI, and 5 comorbidities (diabetes, hypertension, hyperlipidemia, hypertensive heard disease, other heart disease) significantly associated with successful weight loss. At 3 years post-WLS, $49.9 \%$ of patients had successful weight loss. Hypertension, anxiety disorders, and nonalcoholic steatohepatitis were significantly associated with successful weight loss. At 4 years post-WLS, $48.7 \%$ of patients experienced successful weight loss, with race, age, and nonalcoholic steatohepatitis significantly associated with success $(\mathrm{P}<0.05)$.

Conclusion: Our results suggest that a larger number of patient characteristics are associated with successful weight loss at 1 year post-WLS as compared to success at 2 to 4 years post-WLS. The percentage of successful weight loss decreases with time after surgery. These results support the efficacy of WLS in the general population and that there may be a need for sustained support out to 2 years post-WLS to help patients experience optimal postWLS outcomes. 


\section{Symptom Burden Is Lower in Asian and Pacific Islander Patients Admitted to Home-Based Palliative Care but Similar Among Other Racial/ Ethnic Groups}

\author{
Emily Rozema, Eric Haupt, Richard Mularski, Huong \\ Nguyen
}

Kaiser Permanente Southern California, Pasadena, CA

Background: Prior studies suggest that, among patients admitted to home-based palliative care (HBPC), patientreported pain, depression, and overall symptom burden are higher among Black and Hispanic patients. However, these studies were mostly descriptive in nature and did not account for other relevant covariates. The purpose of this analysis is to add to existing knowledge by determining if symptom burden differs between seriously ill White patients and patients of color in a large, ethnically diverse HBPC cohort.

Methods: We drew from baseline data (2019) collected as part of a comparative effectiveness trial of two models of HBPC for patients with serious illness and their family caregivers across two Kaiser Permanente regions. The Edmonton Symptom Assessment Scale (ESAS) was administered to patients or proxy caregivers by research staff or HBPC clinicians. To assess for differences in ESAS total scores at admission to HBPC, we performed a univariate analysis of differences in mean scores and a multivariate median regression analysis across all race/ethnicity groups. Covariates included age, gender, insurance type, income, receipt of medical financial assistance, Charlson comorbidity score, admitting diagnosis, impairment with activities of daily living, do-not-resuscitate code status, hospital utilization in the prior 7 days, treatment arm, proxy response, whether the ESAS was administered by research staff or clinicians, and site.

Results: Characteristics of the 2245 patients were mean age of $78 \pm 13$ years, $52.7 \%$ female, $54.2 \%$ White, $23.4 \%$ Hispanic, $13.5 \%$ Black, 8.4\% Asian/Pacific Islander (PI), and 0.6\% multiracial/other/unknown. In the univariate analysis, mean baseline ESAS scores were comparable across White (29.4 $\pm 14.35)$, Black (29.7 \pm 15.14$)$, and Hispanic (29.3 \pm 15.75$)$ groups but lower among Asian/PIs (25.4 \pm 13.6$)(\mathrm{P}=0.002)$. In the multivariate analysis, mean baseline ESAS score for the Asian/PI group was lower $(-3.5[-6.2,-0.8] ; \mathrm{P}=0.01)$ compared to the White group. There were no significant differences across other race/ethnicity categories.

Conclusion: These findings indicate that, in this integrated health system cohort, while symptom burden was not higher in Black and Hispanic groups, it was lower among Asian/PI patients admitted to HBPC compared to Whites. More research is needed to assess whether access to care, health system factors, geographic factors, or other factors may contribute to previous findings of disparities in symptom burden.

\section{Challenges of Comparative Effectiveness Research in Palliative Care: Lessons From a Pragmatic Trial of Video Consultations in Home- Based Palliative Care}

Carmit McMullen, Huong Nguyen, Susan Wang, Henry Werch, Paula Edwards, Gina Andres, Brian Mittman, Ernest Shen, Lynn Reinke, Richard Mularski

\section{Kaiser Permanente Northwest, Portland, OR}

Background: Comparative effectiveness studies using pragmatic trial designs offer opportunities to generate robust evidence that health systems need about interventions and innovations tested in real-world settings. In palliative care, evidence-based interventions are needed to identify practice innovations for ameliorating the unmet demand for services. Telemedicine has the potential to enhance access, but there is little evidence about telehealth interventions in this setting. Methods: We conducted a pragmatic, cluster-randomized noninferiority trial in two large integrated health systems (HomePal Study). Registered nurses $(\mathrm{n}=111)$ at 14 sites were randomized to standard in-person care vs videovisit supported care. Patients and caregivers were enrolled in the study, which included mixed-methods process evaluation using the NASSS (nonadoption, abandonment, scale-up, spread, sustainability) framework of technology nonadoption. Primary outcomes included symptom burden and caregiving preparedness at 1-2 months.

Results: The study was stopped early due to low video-visit uptake and the health system's decision to expand telehealth to both study arms, the latter of which was incompatible with the randomized design. Maintaining static comparators and randomization of nurses ultimately proved unfeasible. Funding specifications precluded adaptations that we proposed in response to health system needs and constraints. Pivoting to a cluster-randomized step-wedge design would have allowed a staggered rollout of video-enabled care, an approach that is more familiar and amenable to health systems and clinicians. Shifting to an adaptive trial design would have enabled iterative improvements of our intervention as we gained experience with video visits and the technology platforms and incentives for telehealth delivery evolved.

Conclusion: The success of large, multisite pragmatic trials testing complex interventions depends on continued support and engagement of health system stakeholders, ranging from frontline clinicians to executives. Multiyear palliative care trials must do so while serving severely ill patients with acute needs in a rapidly shifting landscape of market pressures and regulatory/payment changes. Our experience offers important lessons, particularly with the unprecedented COVID-19 pandemic - the need to be flexible and nimble with study designs and methodology and the need to implement changes in the face of imperfect or incomplete evidence, as was the case with telehealth. 


\section{COVID-19}

\section{"Home a-Lonely": Understanding Emotional and Social Complexities of Remote Work During the COVID Pandemic}

\author{
Heather R. Walker, Isaac Holyoak \\ University of Utah Health, Salt Lake City, UT
}

Background: As the infection and death rates for COVID-19 continue to soar on a global scale, researchers around the world are coming forward to measure, evaluate, and understand the impacts of this virus. For example, the White House issued a Call to Action on March 16, 2020, to bring forward technology researchers to analyze COVID-19 data. Likewise, academic journals like The Lancet, Global Heart, Journal of Medical Internet Research, and others put out calls for COVID-19-centered research. To meet the increased call for expansive research on the impacts of COVID-19, this research sets out to understand how the shift from oncampus to remote work has been impacting work culture at two University of Utah Health teams.

Methods: This mixed-methods study aims to understand the social and emotional complexities of forced remote work experienced during the COVID-19 pandemic. To do this, a SenseMaker ${ }^{\circledR}$ project was developed and deployed via social media and through two institutional employee lists from University of Utah Health. SenseMaker ${ }^{\circledR}$ is a sensemaking tool that captures both widespread patterns and weak signals in attitudes and mindsets. It requires respondents to visually weigh various relational elements to interpret their experiences around a given topic or phenomenon (Van der Merwe et al, 2019).

Results: Findings reveal a resource-based discrepancy between individuals' coping behaviors and strategies, a distinct polarization in outlook toward remote work, and a feeling of productivity based more on meeting basic demands than on set work hours. Also, this study finds that conversations about mental health are not being had to the extent they are perceived to be needed.

Conclusion: When the Work-Home Resources Model (Ten Brummelhuis, 2012) is applied to contextualize experiences with forced remote work during a pandemic for health teams, it becomes evident that mental health needs to be strongly emphasized. Findings from this study complicate understandings of productivity and provoke further discussion of how health systems might best approach employee work and health in the future.
Disparity of Seroprevalence of SARS-CoV-2 IgG Antibodies in a Large Midwestern Health System

\author{
Veronica Fitzpatrick, Anne Rivelli, Jon Richards
}

Advocate Aurora Health, Downers Grove, IL

Background: Recent research has suggested widespread disparity in COVID-19 distribution among racial and ethnic minorities, especially Black and Hispanic populations. In all cases, Black persons are more likely to contract COVID-19 and are twice as likely as White persons to die of related complications. Black and Hispanic persons may be at greater risk of exposure to COVID-19 because they are more likely to be essential workers and also tend to live in densely populated areas and multigenerational households The objective of the study was to contribute to current research on distribution of exposure to SARS-CoV-2 immunoglobulin $\mathrm{G}$ (IgG) by describing disparities across baseline demographic and clinical role factors among a large cohort of health care workers employed by one of the largest Midwestern health systems in the United States.

Methods: This cross-sectional study collected 16,233 SARS-CoV-2 IgG serum samples between June 8, 2020, and July 10, 2020, from a convenience sample of Illinoisand Wisconsin-based adult health care workers. Positive presence of SARS-CoV-2 IgG antibodies was detected using the ARCHITECT assay ("SARS-CoV-2 IgG assay"), developed in collaboration with Abbott Laboratories and approved for use by the U.S. Food and Drug Administration. Study data included SARS-CoV-2 IgG assay result and baseline factors of interest (age, sex, race, ethnicity, clinical role category, zip code). Crude and adjusted relative odds were generated to describe disparities.

Results: Among the 16,233 total participants, 622 (3.83\%) had positive SARS-CoV-2 IgG. Crude and adjusted odds revealed age, race, ethnicity, and clinical role disparities at $\mathrm{P}<0.0001$. Participants $32-82$ years old (vs age 18-31) had lesser adjusted odds of positive IgG (0.54-0.66). Black, Asian, and mixed-race participants (vs White participants) had greater adjusted odds of positive $\operatorname{IgG}(3.86,1.87$, and 1.99 , respectively). Hispanic (vs non-Hispanic) participants had 1.80 times greater adjusted odds of positive IgG. COVID-clinical (vs nonclinical) participants had 1.86 times greater odds of positive IgG.

Conclusion: This study highlights the disparity in distribution of the SARS-CoV-2 virus by age, race, ethnicity, and clinical role category. Generally, positive SARS-CoV-2 IgG was detected more among health care workers who were youngest, non-White, Hispanic, and working in COVIDdesignated clinical units. 


\section{Widening Cancer Care Disparities in the Adoption of Telemedicine During COVID-19: Who Is Left Behind?}

Su-Ying Liang, Michael Richardson, Monique de Bruin, Tony Chen, Natalia Colocci, Allison Kurian, John Chan

\section{Sutter Health, Palo Alto, CA}

Background: COVID-19 has reshaped how care is delivered - in person, virtually, or postponed. While telemedicine has served as an alternative way to access care since the beginning of the pandemic, relatively little is known about its utility in cancer care and possible disparities in care delivery. We examined the impact of COVID-19 on volume of oncology services and adoption of telemedicine across patient subgroups.

Methods: Data were obtained from the electronic health record of a multispecialty health system serving a racially/ethnically diverse patient population in Northern California. The study cohort included 52,985 patients who had at least 1 oncology encounter from January 2019 to August 2020. To compare COVID-19-related changes in volume of encounters, we assessed the yearto-year variability for appointment cancellation, office visits, and cancer screening procedures. Multivariate logistic regression was performed to examine variability in use of telemedicine (video visits) by specific patient characteristics (sex, age, race/ethnicity, and language), controlling for comorbidity, insurance type, and preCOVID-19 health care utilization.

Results: Compared to the pre-COVID-19 monthly averages in 2019, oncology appointment cancellation rates increased by $73 \%$ in March 2020 . Volume of services substantially declined in 2020 (by $66 \%$ for oncology office visits, by $92 \%$ for mammograms, and by $95 \%$ for colonoscopies in April). Use of telemedicine for oncology started in midMarch 2020, grew to 55\% of all scheduled visits in April, and remained at 38\% in August. We focused on variability by specific patient subgroups when telemedicine was used prevalently in April 2020. On regression analysis, Hispanics were significantly less likely to use telemedicine than Whites (odds ratio: 0.62, 95\% CI: 0.51-0.76). Patients who were older (odds ratio: 0.62, 95\% CI: 0.49-0.78) or who required interpreting services (odds ratio: 0.44 , 95\% CI: 0.32-0.61) were significantly less likely to use telemedicine than patients who were younger or did not need an interpreter.

Conclusion: Overall office visits decreased significantly during the COVID-19 pandemic. Uptake of video visits was uneven across patient subgroups and lowest among older, Hispanic patients and those who required interpreting services. Future studies are needed to better understand the barriers to and preferences for transition to telemedicine.
Disparities in Outpatient and Telehealth Visits During the COVID-19 Pandemic in a Large Integrated Health Care Organization

\section{Lei Qian, Stanley Xu, Lina Sy, Sungching Glenn,} Vennis Hong, Denison Ryan

Kaiser Permanente Southern California, Pasadena, CA

Background: Dramatic decreases in outpatient visits and sudden increases in telehealth visits were observed after the start of the COVID-19 pandemic. But it is unclear whether these changes differed by patients' demographics and socioeconomic status. The objective of this study was to assess the impact of the pandemic on outpatient and telehealth visits by demographics and household income level in a diverse patient population.

Methods: We calculated weekly rates of outpatient and telehealth visits (telephone or video) by age, sex, race/ ethnicity, and neighborhood household income among members of Kaiser Permanente Southern California (KPSC) during January 5, 2020-October 31, 2020, and the corresponding period in 2019. We estimated the percent change by comparing visit rates during the pandemic period to the prepandemic period (January 1-March 7, 2020) using Poisson regression models, adjusting for seasonality. We examined if the changes differed by age, sex, race/ethnicity, and household income level.

Results: Among 4.55 million KPSC members enrolled in January 2020 , about $15 \%$ were $\geq 65$ years old, $49 \%$ were female, $39 \%$ were Hispanic, and $8 \%$ lived in an area of median household income less than $\$ 40,000$. Across all subgroups, outpatient visit rates decreased while telehealth visit rates increased due to the pandemic. Among age groups, the 65 and older population had the smallest increase in telehealth visits: $1.5 /$ person-year during the prepandemic period vs 3.9/person-year in October 2020 (a 161\% jump, 95\% CI: 158\%-164\%). During the same periods, across racial/ethnic groups, Hispanic individuals had the largest increase in telehealth visits $(220 \%, 95 \%$ CI: $217 \%-223 \%$ ); across income levels, telehealth visits in the low-income group increased the most $(220 \%, 95 \%$ CI: $214 \%-227 \%$ ). The rate of combined outpatient and telehealth visits in the low-income group returned to prepandemic levels $(0.7 \%, 95 \%$ CI: $-0.2 \%-1.6 \%)$ by October 2020.

Conclusion: While the 65 years and older population had the smallest increase in telehealth visits, the Hispanic group and the low-income group had the largest increase in telehealth utilization possibly due to the low cost and flexibility of location and time. 
Impact of the COVID-19 Pandemic on Hepatitis C Screening in Kaiser Permanente Mid-Atlantic States

\author{
Cabell Jonas, Michael Horberg, Eric Watson, Aniecia \\ Gunn, Jared Williamson, Anna Dinucci
}

Kaiser Permanente Mid-Atlantic States, Rockville, MD

Background: Hepatitis $\mathrm{C}$ virus (HCV) is a bloodborne infectious disease. An estimated 2.4 million Americans are living with HCV; half of these individuals may not know they are infected. In 2014, the U.S. Preventive Services Task Force (USPSTF) recommended individuals of babyboomer age (born 1945-1965) and those otherwise at risk be screened one time for HCV. In March 2020, the USPSTF expanded its recommendation to include all patients 18-79 years of age. In Maryland, Virginia, and Washington, DC, COVID-19 cases began to rise beginning in March 2020. In the months following, Kaiser Permanente Mid-Atlantic States (KPMAS) clinics began to reduce services and limit hours, and patients began delaying nonurgent care. COVID-19 cases persisted in the region throughout 2020 and into the present. Herein, we describe the resulting impact of the first year of the COVID-19 pandemic on HCV screening in the KPMAS health system.

Methods: The HCV Pathway is a standardized HCV screening program designed to improve screening rates. The HCV Pathway became the standard for adult HCV screening in March 2019. Hepatitis C screening through the HCV Pathway, facility closures, and COVID-19 infection rates were examined during the time periods of March 2019 to February 2020 (pre-COVID-19 pandemic) and March 2020 to February 2021 (during COVID-19 pandemic). Tested patients were examined by age, sex, race/ethnicity, service area, insurance type, and documented social history (intravenous drug use, men having sex with men).

Results: HCV testing rates were significantly reduced during key months of the COVID-19 pandemic in 2020. The downward trend in $\mathrm{HCV}$ testing seen from March to July 2020 was reversed by an operational change in August 2020 that expanded HCV testing to patients 18-79 years old via electronic health record-based health maintenance topics and best practice alerts. As a result of the operational change, HCV screening rates increased above 2019 rates temporarily. The deactivation of the best practice alerts in December 2020 resulted in a stabilization of HCV testing into 2021 to pre-COVID 2020 levels.

Conclusion: $\mathrm{HCV}$ screening rates were significantly reduced during key months of the COVID-19 pandemic; however, the implementation of electronic health recordbased alerts stabilized testing rates to at or above preCOVID-19 pandemic levels.
Medication Adherence During the COVID-19 Pandemic: Preliminary Data From the Patient Perspective

JoAnn Sperl-Hillen, Lilian N. Chumba, Patrick J. O'Connor, Pamala A. Pawloski, Jeanette Y. Ziegenfuss

HealthPartners, Bloomington, $M N$

Background: This study aimed to share preliminary descriptive survey results from patients with potential medication adherence issues on how they changed taking or filling medications during the COVID-19 pandemic.

Methods: As part of a larger trial of a clinical decision support intervention, patients identified as potentially nonadherent to medications were contacted by telephone to complete a survey in the weeks after a clinical encounter. The 10-minute survey asked patients about their recent experiences with taking and filling medications during the COVID-19 pandemic. Responders received a \$10 thank you incentive. Patients surveyed met criteria at a clinical encounter for not being at clinical goal for either glycated hemoglobin or blood pressure or if they were on a statin and had a low adherence score $(<80 \%)$ to a medication in that domain. The adherence scores were Epic-derived proportion of days covered (PDC) calculations using Surescripts dispense data in the last 6 months, and only scores rated with high data confidence were included.

Results: The survey is currently in the field, with a $50 \%$ anticipated response rate after all reach attempts are completed. To date, a total of 123 individuals have completed the survey; $13 \%$ of respondents noted that they had changed how they take or fill prescriptions for medicines due to the COVID-19 pandemic. Of these patients, $53 \%$ indicated they were taking their medication more regularly than before and $27 \%$ indicated they were taking medication less regularly or having difficulty taking their medication as prescribed. None of the individuals reported having trouble refilling their medication, $27 \%$ refilled their medicine sooner or more often to make sure they didn't run out, $27 \%$ changed to refill their prescription by mail order, and $20 \%$ indicated they were more concerned about the cost of their medications.

Conclusion: Of patients not achieving care goals and identified with potential adherence issues, less than onefifth reported that the COVID-19 pandemic changed how they took or refilled their medication. Of patients who did make changes, more than half reported taking medications more regularly than before. They reported no trouble obtaining refills, but cost was a concern for some. 
Influenza and COVID-19 Vaccination Intent, Knowledge, and Attitudes in Symptomatic Patients

Michelle Henninger, S. Bianca Salas, Phil Crawford, Judy Donald, Holly Groom, Allison Naleway

\section{Kaiser Permanente Northwest, Portland, OR}

Background: The COVID-19 pandemic has led to an increased emphasis on seasonal influenza (flu) vaccination with the goal of alleviating strain on stretched health systems. It is unknown how the pandemic has impacted flu vaccination intention and coverage during the 2020-2021 flu season. Further, with COVID-19 vaccines now being administered, more data is needed on people's intent to be vaccinated and their knowledge and attitudes about the COVID-19 vaccines.

Methods: As part of a larger study on SARS-CoV-2 and flu testing, we identified adult members of Kaiser Permanente Northwest, living in the Portland-Vancouver metropolitan area, with a recent telehealth visit consistent with COVID-19 or flu symptoms and an incomplete laboratory order for flu or SARS-CoV-2 testing. Participants completed an eligibility survey assessing intention to vaccinate for flu and COVID-19 and an enrollment survey assessing knowledge and attitudes about the COVID-19 vaccine.

Results: Since September 2020, we have invited 3762 members to participate; $647 \quad(17 \%)$ completed the eligibility survey and $368(10 \%)$ completed the enrollment survey. Most (62\%) reported receiving the flu vaccine during the previous (2019-2020) season. As of January $23,2021,46 \%$ reported having already received the flu vaccine during the current (2020-2021) season. Of those not vaccinated, $67 \%$ reported at least moderate intent to receive the flu vaccine. Only $2 \%$ reported having already received the COVID-19 vaccine. Of those who had not, $86 \%$ reported at least moderate intent of receiving the COVID-19 vaccine. Those who did not plan to get vaccinated for COVID-19 (14\%) were significantly more likely than those who did to be concerned about vaccine side effects and to believe the vaccine could give them COVID-19. They were significantly less likely to believe they were at risk of getting COVID-19, that their primary care provider would recommend the vaccine, or to trust federal vaccination recommendations.

Conclusion: Most participants reported receipt or intent to receive the 2020-2021 flu vaccine, and intent to receive the COVID-19 vaccine was even higher than that for the flu vaccine. Patient education focusing on the safety of the COVID-19 vaccine, and risks of not being vaccinated, are needed to address vaccine hesitancy.
How Can Artificial Intelligence Be Leveraged to Effectively Manage Pandemics? A Scoping Review

Ania Syrowatka, Masha Kuznetsova, Ava Alsubai, Adam L. Beckman, Paul A. Bain, Kelly J. Thomas Craig, Jianying $\mathrm{Hu}$, Gretchen P. Jackson, Kyu Rhee, David W. Bates

Brigham and Women's Hospital / Harvard Medical
School, Boston, MA

Background: COVID-19 has had a profound impact on all aspects of society, and future pandemics are sure to follow. Artificial intelligence represents a valuable tool that could be used to effectively manage pandemics. The objective of this scoping review was to identify use cases for involving artificial intelligence for pandemic preparedness and response from the peer-reviewed, preprint, and grey literature.

Methods: We identified studies by searching 5 databases (PubMed, Embase, Web of Science, IEEE Xplore, ACM Guide to Computing Literature) and 2 preprint servers (medRxiv, bioRxiv). We used a structured Google search to identify grey literature. The data synthesis had two parts: an in-depth review of studies that leveraged machine learning, and a limited review of studies that applied traditional modeling approaches such as compartmental models. Machine learning applications from the in-depth review were categorized into use cases related to public health and clinical practice and narratively synthesized. Data sources used for model development and types of machine learning well suited for each use case were summarized.

Results: 183 articles met the inclusion criteria for the indepth review and reported on the development of machine learning algorithms or tools in response to COVID-19, the 2009 H1N1 pandemic influenza, severe acute respiratory syndrome (SARS), or hypothetical pandemics. Six key use cases were identified: forecasting infectious disease dynamics and effects of interventions; surveillance and outbreak detection; real-time monitoring of adherence to public health recommendations; real-time detection of influenzalike illness; triage and timely diagnosis of infections; and prognosis of illness and response to treatment. Data sources and types of machine learning that were useful varied by use case. Application of machine learning was still at the research or developmental stage for most use cases. Overall, 1173 articles reported on traditional modeling approaches and were included in the limited review, which served to highlight additional areas where machine learning could be leveraged for pandemic preparedness and response.

Conclusion: Important machine learning-based solutions have been developed in response to pandemics, but few were optimized for practical application early in the COVID-19 pandemic. These findings can support policymakers, clinicians, and other stakeholders in prioritizing research and development to support operationalization of artificial intelligence for future pandemics. 
Characteristics, Clinical Care, and SARS-CoV-2 Testing of Patients Utilizing e-Visit Encounters for COVID-19-Like IIIness, March-October 2020

Holly Groom, Phil Crawford, Michelle Henninger, S. Bianca Salas, Judy Donald, Lenee Blanton, Eduardo Azziz-Baumgartner, Allison Naleway

\section{Kaiser Permanente Northwest, Portland, OR}

Background: Kaiser Permanente Northwest (KPNW) health system implemented an online COVID-19 electronic visit (e-visit) in February 2020. This self-administered screening tool allows patients to report COVID-19-like illness (CLI) symptoms and exposures; providers review information and recommend a clinical course of action within 24 hours. We describe the demographics of members using the e-visit tool and subsequent clinical care recommendations and laboratory outcomes.

Methods: Using KPNW electronic health records, we identified CLI e-visits among adult members from March through October 2020. We calculated the proportion of e-visits associated with subsequent health care visits (categorized by most intensive care type received: inpatient/ emergency room (ER), ambulatory in-person, virtual, labonly); SARS-CoV-2 lab orders; and positive SARS-CoV-2 test results. We compared demographics and prevalence of conditions established as increasing the severity of COVID-19 illness (eg, body mass index [BMI] of $\geq 40$, type 2 diabetes) between those with e-visits to general KPNW members (with 1 or more health system encounters from November 1, 2019, through October 31, 2020) and between those who were and were not referred for SARS-CoV-2 testing following a CLI e-visit.

Results: We identified 24,674 CLI e-visits. The rate of e-visits was highest among members 25-34 years of age (294 e-visit encounters per 1000 members), double that of non-CLI encounters in this age group in the prior 12 months (143 per 1000). Of all CLI e-visits, $86 \%(n=21,162)$ led to subsequent encounters, among those were inpatient/ER ( $2 \%)$, ambulatory in-person (65\%), virtual telephone/video $(31 \%)$, and lab-only (2\%). From May through October (when SARS-CoV-2 testing was consistently available), $77 \%$ of e-visits resulted in lab orders. Overall, $4.3 \%$ of tests were positive for SARS-CoV-2 (weekly range: $0 \%-10.3 \%$ ). Demographics of members with and without test orders were similar, as was the prevalence of high-risk conditions such as BMI $\geq 40$ (6.3\% vs $7.6 \%$, respectively) and type 2 diabetes $(6.3 \%$ in both groups).

Conclusion: Many KPNW members, especially those 2535 years old, made use of the CLI e-visit. Most were referred for subsequent care and/or SARS-CoV-2 testing, although only a small proportion of tests were positive. Although symptom severity was not obtained, similar rates of highrisk conditions were observed between those who did and did not receive lab orders.
Heterogeneity in COVID-19 Patients at Multiple Levels of Granularity: From Biclusters to Clinical Interventions

Suresh K. Bhavnani, Erich Kummerfeld, Ravi Radhakrishnan, Christopher Tignanelli

University of Texas Medical Branch, Galveston, TX

Background: Several studies have shown that COVID-19 patients with prior comorbidities have a higher risk for adverse outcomes, resulting in a disproportionate impact on older adults and minorities that fit that profile. However, although there is considerable heterogeneity in the comorbidity profiles of these populations, not much is known about how prior comorbidities co-occur to form COVID-19 patient subgroups and their implications for targeted care.

Methods: We used bipartite networks to quantitatively and visually analyze heterogeneity in the comorbidity profiles of COVID-19 inpatients, based on electronic health records from 12 hospitals and 60 clinics in the greater Minneapolis region. This approach enabled the analysis and interpretation of heterogeneity at 3 levels of granularity: 1) cohort level to identify commonly occurring phenotypes in COVID-19 patients; 2) subgroup level to measure the risk of each phenotype; and 3) patient level to examine which patients can use interventions designed for the subgroup and which require individualized interventions.

Results: The results revealed statistically and clinically significant results at each level of granularity. 1) Cohort: The algorithm identified 4 biclusters consisting of patient subgroups and their most frequently co-occurring comorbidities, which was statistically significant compared to 1000 random permutations of the data (COVID-19 Q: 0.22 , random median $\mathrm{Q}: 0.19 ; \mathrm{P}<0.001$ ). The 4 biclusters were qualitatively inferred as representing distinct phenotypes: cardio-renal, coagulapathy liver disease, diabetes-obesity-psychosis, and hypertensive-electrolyte. 2) Subgroup: Each patient subgroup had different combinations of significant associations with adverse outcomes compared to the controls. For example, the cardio-renal had significant odds ratios for all 3 adverse outcomes. These associations to adverse outcomes enabled the clinicians to design triage strategies targeted to each phenotype. 3) Patient: A majority of the outlier patients in each subgroup had comorbidities within their biclusters. However, the generic treatment plans for the entire subgroup were often not appropriate, suggesting customization of treatment for such patients.

Conclusion: The results suggest that each level of granularity in the bipartite network analysis provided distinct treatment and triage insights based on the cooccurrence of comorbidities. A critical limitation of the current research is that we analyzed only one dataset, and our future research will test the replicability of these results in another COVID-19 dataset. 


\section{Impacts of COVID-19 on the Peer Recovery Specialist Role}

Morgan Anvari, CJ Seitz-Brown, Jessie Spencer, Sara Abdelwahab, Megan Mulheron, Jessica Magidson, Julia Felton

\section{University of Maryland, College Park, College Park, MD}

Background: The opioid epidemic and COVID-19 have both disproportionally affected underserved minority communities. Peer recovery specialists (PRSs), individuals who have lived substance use experience, are uniquely equipped to serve low-income, ethnoracial minority clients who have been doubly burdened by a lack of access to substance use and COVID-19 treatment and resources. As the health system relies on PRSs in a variety of departments, it is important to understand how they are navigating the pandemic. This research aimed to learn 1) the impact of COVID-19 on the PRS role/experience, and 2) how experience, training, and supervision have or have not prepared PRSs for these challenges.

Methods: PRSs $(n=15)$ from Maryland and New Jersey were recruited via snowball sampling for semi-structured, qualitative interviews. Thematic, rapid qualitative analysis was conducted to examine themes related to the study objectives.

Results: PRSs described numerous challenges and shifts in their role in face of the pandemic. These included logistic challenges, such as an inability to refer clients to usual resource centers, assistance programs, and treatment programs due to COVID-19-related closures and policies. PRSs also described a shift in their role, with an increased focus on patient basic needs, and an increase in communication and follow-up with clients to combat a perceived loss of interpersonal connection. Many PRSs explained that while their training did not prepare them for a pandemic, their shared substance use experience, empathy, and flexibility uniquely prepared them to support their clients. Additionally, participants noted that a key part of the PRS role is helping clients overcome barriers to treatment, making PRSs well equipped to assist clients as the pandemic creates novel treatment barriers.

Conclusion: COVID-19 has posed unique challenges to and brought on shifts in the PRS role. However, factors unique to the PRS role, such as shared experience and helping clients overcome barriers to treatment, have prepared PRSs to assist clients during the pandemic. Further research may look into how COVID-19 has affected the PRS role over time and how novel trainings may support PRSs during the pandemic. Lessons learned during the pandemic can inform future developments regarding the PRS role.
Impact of Health Care Provider Recommendations on COVID-19 Vaccination Intent

Kimberly A. Fisher, Ngoc Nguyen, Hassan Fouayzi, Sonal Singh, Sybil Crawford, Kathleen M. Mazor

\section{Meyers Primary Care Institute, Worcester, MA}

Background: A substantial proportion of the U.S. population is hesitant to be vaccinated against COVID-19. The importance of a health care provider recommendation in promoting vaccination is well established for the influenza and human papillomavirus vaccines. We tested a series of health care provider recommendations among COVID-19 vaccine-hesitant individuals to identify elements that increase intent to be vaccinated.

Methods: We conducted an online survey using the Prolific panel in January 2021, oversampling Black and Latino panel members. Participants were classified as vaccine-hesitant if they responded "no" or "not sure" to the question "Do you intend to be vaccinated against COVID-19?" Vaccinehesitant participants were asked to imagine they were seeing their doctor for an annual exam, at the end of which the doctor states that the COVID-19 vaccine is available and that the patient is eligible to receive it at that visit. Participants were randomly assigned to receive either an "elective" recommendation (control) or 1 of 4 strong recommendations (intervention), followed by reassessment of vaccination intent. All messages included a statement that the vaccine is very safe and very effective. In the control message, this was followed by "What do you think?" (ie, an elective recommendation), whereas the intervention messages included an explicit recommendation (ie, "I recommend that you get it"). The intervention messages differed in the strategy used to encourage vaccination (personal physician review of safety data, comparison to the flu shot, statement that millions of people have already received it, emphasis on protecting others).

Results: The 756 vaccine-hesitant respondents were $60.2 \%$ female, 44.2\% Black, 22.4\% Latino, and 33.4\% White, with a mean age of 35.6 years (range: 18-73). Vaccinehesitant participants were significantly more likely to accept vaccination after receiving a strong health care provider recommendation than participants who received an elective recommendation $(19.3 \%$ vs $9.9 \% ; \mathrm{P}<0.015)$. There were no statistically significant differences between the 4 strong recommendations.

Conclusion: A strong explicit recommendation from a health care provider significantly increases the likelihood that a COVID-19 vaccine-hesitant individual will accept vaccination compared with an elective-style recommendation. This finding highlights the importance of health care provider outreach and delivery of strong recommendations to foster COVID-19 vaccine acceptance. 


\section{Reasons for COVID-19 Vaccine Hesitancy and Conditions for Acceptance}

Kimberly A. Fisher, Ngoc Nguyen, Hassan Fouayzi, Sonal Singh, Sybil Crawford, Kathleen M. Mazor

\section{Meyers Primary Care Institute, Worcester, MA}

Background: The severe acute respiratory syndrome coronavirus 2 (SARS-CoV-2) and associated coronavirus disease 2019 (COVID-19) pandemic have been devastating. In the United States, more than 28 million people have been infected, resulting in more than 500,000 deaths. The approval of effective COVID-19 vaccines is reason for optimism, but realizing the full benefits of vaccines will require widespread uptake. Overlap between factors associated with COVID-19 disease and COVID-19 vaccine hesitancy threaten to exacerbate existing health disparities. We investigated reasons for and associations with vaccine hesitancy to inform strategies for promoting COVID-19 vaccination among vulnerable populations.

Methods: In January 2021, we conducted an online survey using the Prolific panel, oversampling Black and Latino panel members. Respondents received $\sim \$ 1.50$ consistent with Prolific recommendations. This abstract describes reasons for vaccine hesitancy and the relationship between vaccine hesitancy, vaccine knowledge, and numeracy.

Results: The analytic sample included 1706 respondents; $53.3 \%$ identified as female; $30.2 \%$ identified as Latino, $32.5 \%$ as Black, and $34.8 \%$ as White; $24.4 \%$ reported a high school education or less; and the mean age was 34.5 years (range: 18-76). Asked if they would be vaccinated for COVID-19, 24.3\% responded "no" and 20\% responded "not sure." We categorized both responses as vaccinehesitant. The most common reasons for vaccine hesitancy were concern about vaccine safety (34.3\%), speed of vaccine development (20.2\%), insufficient testing (14.7\%), and distrust of COVID-19 vaccines (10.2\%). A majority of hesitant respondents $(54.3 \%)$ reported they would get vaccinated if the vaccine was in widespread use; $50.2 \%$ would get vaccinated if the COVID-19 vaccine was as safe as the flu shot; and $47.2 \%$ would get vaccinated if someone in their household was at risk. Vaccine-hesitant respondents scored lower than nonhesitant respondents on vaccine knowledge ( 4.8 of 7 vs 5.6 of 7 correct; $\mathrm{P}<0.001$ ) and relied less on numbers when making health decisions $(64 \%$ vs $84 \% ; \mathrm{P}<0.001)$.

Conclusion: Overall, respondents who intend to get vaccinated had more accurate vaccine-related knowledge and reported greater reliance on numbers in making health decisions. These findings suggest that messaging to vaccinehesitant adults should provide evidence of vaccine safety using narrative and comparisons to familiar, accepted health practices (ie, flu shots) rather than statistics.
DATA SCIENCE, INFORMATICS, AND DATA MODELS

\section{Computerized Ejection Fraction Extraction Algorithm Development and Validation}

Xiangyi Xu, Xiaowei (Sherry) Yan, Wendi Knapp, Souvik Das, Anthony Sturzu, Kimberly Buss

Sutter Health, Walnut Creek, CA

Background: Left ventricular ejection fraction (EF) is a diagnostic indicator for the classification of heart failure and is used in treatment. However, EF is documented with variations in free-text reports, which hinders its utilization. We developed a regular-expression-based algorithm to extract EF values from echocardiogram reports and conducted validation.

Methods: We conducted 3 phases of chart review to validate the algorithm. The extracted $\mathrm{EF}$ value and $\mathrm{EF}$ quality referred to domains where EF was extracted from, and the algorithm was refined based on validation findings. In the first phase, 112 randomly selected echocardiogram reports were reviewed by a physician and compared to algorithm-retrieved EF. In the second phase, 512 randomly selected reports, stratified by departments, were reviewed by physicians $(n=7)$. Each report was reviewed by 2 physicians. In the final validation phase, we trained 10 physicians in charting review results, and they reviewed 1001 randomly selected reports from January 1, 2019, to December 31, 2019. We evaluated the interreviewer agreement on outcomes and also compared the algorithmderived values with the "gold standard," defined as the agreed-upon EF value between reviewers.

Results: A regular-expression algorithm was developed based on keywords list provided by clinicians and initial mining of 280,000 echocardiogram reports. In phase 1, both the physician and the algorithm identified EF values from 88 of 112 reports $(78.6 \%)$, showing $100 \%$ consistency of EF-value identification fidelity. Among those 88 reports, the accuracy of EF value extracted by the algorithm was $97.5 \%$. In phase 2, interreviewer consistency was $93.9 \%$ for EF value and $82.5 \%$ for EF quality. Compared to gold standard on 499 reports, the algorithm accuracy of EF value was $97.2 \%$. In the final phase, interreviewer consistency for EF value remained high (97.2\%) and was improved for EF quality $(92.7 \%)$. Algorithm accuracy improved to $98.8 \%$.

Conclusion: Cardiovascular domain knowledge is crucial in developing algorithms for extracting EF values. The 3-phase validation has ensured the algorithm's accuracy and validity. The algorithm was implemented to extract EF from all historical echocardiogram reports, and discrete EF will be available in electronic health records for use in the system going forward from April 2021. 


\section{Updating Out-of-Date Virtual Data Warehouse Tumor Variables to Align With Current AJCC8 Standards}

\author{
Rick Krajenta, Lois E. Lamerato
}

Henry Ford Health System, Detroit, MI

Background: AJCC Cancer Staging Manuel, 8th Edition was released in October 2016 to be implemented for cases diagnosed on or after January 2018. This new version had its companion data transmission system upgraded to Version 18 of the North American Association of Central Cancer Registries (NAACCR) standards (NAACCR is now on Version 21). While the AJCC manual establishes rules and data elements for staging, prognostic indicators, and treatment, NAACCR creates format for data transmission to central registries and regulatory agencies. We describe the changes associated with the release of AJCC8 and suggest a data strategy for updating HCSRN's current Virtual Data Warehouse (VDW).

Methods: The new NAACCR has 11 fields retired, 579 fields revised, and 223 new fields. The retired category doesn't include the revisions when data were maintained; 25 site-specific factor (SSF1-SSF25) fields were exploded out to individual columns and no longer populated. The current VDW will need to include STORE fields while maintaining FORDS fields SSF1-25 for historic data. Prognostic factors and posttherapy fields have been added to some topological and/or histologic types as components of staging. Radiation treatment has been completely revised. The new data are very difficult to use for populating current VDW, as neither the NAACCR extract nor local tumor registry conform to VDW definitions. Similarly, current fields will need to hold historic data while new fields should be added. Consequently, the existing VDW has missing data due to not being updated to current national data standards.

Results: Currently at Henry Ford Health, new data items that don't have VDW definition are stored in an ancillary table. This could be a possible template for expanding variables to contain these 802 NAACCR variables. The ancillary tables could link to a core table in a star data architecture. No attempt to recode new radiation treatment to current VDW specs was done at Henry Ford Health, though a similar process as to that for SSF can be implemented.

Conclusion: Although the TUMOR work group has been on hiatus during implementation of this standards upgrade, we need to do something to keep this dataset up to date. This ancillary table has the potential for storage of the existing SSF while providing a flexible structure for additional factors as they are developed.
Generating an Observational Health Data Sciences and Informatics OMOP Data Model from HCSRN's Virtual Data Warehouse

\section{John Weeks}

Kaiser Permanente Washington, Seattle, WA

Background: Over the last 5 years, hundreds of millions of dollars have been granted to research programs that use the Observational Health Data Sciences and Informatics (OHDSI) OMOP common data model. Kaiser Permanente Washington Health Research Institute (KPWHRI) now has a process for generating an OHDSI implementation from our research data warehouse for any given cohort of patients. In 2015, the Precision Medicine Initiative (All of Us) Program was passed by U.S. Congress with an initial funding of $\$ 130$ million; the project decided to harmonize data for analyses across health care organizations using the OHDSI OMOP common data model. KPWHRI used HCSRN's Virtual Data Warehouse (VDW) to create a limited version of OHDSI OMOP for the eMERGE project. That project was improved and moved into a GitHub project that is now available to everyone.

Methods: We created an SAS data file that contained the VDW standard codes and code descriptions. Then, by leveraging the OHDSI OMOP Standard Vocabularies data, we created a mapping from HCSRN VDW codes into OHDSI OMOP standard codes. At KPWHRI we call the tables that provide VDW codes and descriptions Research Code Management tables. We use these code management tables to generate the selected patient data needed to generate the OHDSI OMOP database.

Results: We tested the results of the OHDSI OMOP that we generated by leveraging the cohort definitions generated for the OHDSI OMOP as part of the eMERGE project. The code generated by the OHDSI Atlas application and stored in the PheKB cohort definitions ran without error in our OHDSI OMOP data model. We compared the results of a previously defined VDW cohort with the same requirements, and the patient list was the same for both independently defined cohorts.

Conclusion: The HCSRN is in a great position to leverage the data that they have been curating for almost 2 decades in large National Institute of Health and National Cancer Institute grants that require the use of OHDSI OMOP. KPWHRI is offering a solution through GitHub and welcoming the community to use the software and participate in improving it for our entire network. 


\section{Large Variation in Utilizing Electronic Health Records for Clinicians During Video Visits}

\author{
Xiaowei (Sherry) Yan, Zijun Shen, Hannah Husby, J.B. \\ Jones
}

\section{Sutter Health, Walnut Creek, CA}

Background: Measuring how the electronic health record (EHR) is used for clinic visits has led to insights about how to reduce EHR burden and improve workflow, user interface, and experience. During the COVID-19 pandemic, video visits (VV) have seen rapid adoption across almost all specialties as an alternative to in-person visits (IPV). It is unknown if and how EHR use by clinicians differs during VV and how this varies by specialty.

Methods: We randomly selected 5 individual days from March 1 to April 30 in 2020 and extracted EHR audit logs for all completed outpatient visits. We analyzed clinicians' EHR utilization time by aggregating time spent in EHR activities for each encounter and estimated each clinician's total EHR time and time before and after check-in. We compared clinician's mean EHR time for VV by specialty and compared EHR time for VV to IPV for the same specialty.

Results: Audit log data for 17,369 completed VV and 53,223 IPV were extracted and analyzed. Clinicians accessed the EHR before patient check-in significantly more for VV than IPV (50.9\% of the time vs $23.7 \%$ ). On average, clinician's EHR time per encounter was similar between VV (32.9 minutes, standard deviation [SD]: 28.3) and IPV (32.9 minutes, SD: 31.6). However, clinician's EHR per encounter varied significantly by specialty for VV, from 19 minutes (SD: 18.9) in nephrology to 60.1 minutes (SD: 44.7 ) in integrative medicine. Clinician's EHR time differed significantly between VV and IPV for some specialties: in rheumatology and psychiatry, EHR time for IPV was 17.2 minutes longer than $\mathrm{VV}$; and it differed by 7.1 minutes in internal and family medicine, which account for $42 \%$ of VV. In contrast, in integrated medicine, EHR time was twice as long for VV (mean: 60.1 minutes) than IPV.

Conclusion: EHR use during VV varies significantly across specialties. There is a significant difference in duration of EHR use between VV and IPV within the same specialty. EHR time for clinicians is significantly longer for IPV than for VV in primary care encounters. Incorporating VV into a primary care provider's daily schedule might be an opportunity to address clinician burnout by reducing EHR work.
Can Claims Data Accurately Predict Preoperative Body Mass Index Among Bariatric Surgery Patients? A Comparison of Machine Learning Approaches

Jenna Wong, Xiaojuan Li, David Arterburn, Adee Kennedy, Dongdong Li, Rui Wang, Sengwee Toh

Harvard Medical School and Harvard Pilgrim Health Care Institute, Boston, MA

Background: Lack of body mass index (BMI) measurements hinders use of claims databases in obesity research. In bariatric surgery populations, the ability of claims data to accurately predict preoperative BMI, a potential confounder of surgical outcomes, could further facilitate use of claims data in comparative effectiveness and safety studies of bariatric procedures.

Methods: We used the OptumLabs ${ }^{\circledR}$ data warehouse (January 2011-June 2018), containing linked de-identified claims and electronic health record (EHR) data, to identify patients $\geq 18$ years of age who underwent a new bariatric surgery. Eligible patients had a preoperative BMI recorded in the EHR $\leq 30$ days before the index procedure. We constructed 72 features describing patients' demographics, diagnoses, medications, and previous hospitalizations based on linked claims data $\leq 6$ months before surgery and applied 6 machine learning techniques using minimal human guidance to predict preoperative BMI as a continuous value $\left(\mathrm{kg} / \mathrm{m}^{2}\right)$. We tuned and trained the models on $90 \%$ of patients whose index procedure occurred in 2011-2017. We assessed model performance on the remaining 10\% of patients in 2011-2017 (concurrent) and all patients with an index procedure in 2018 (prospective).

Results: Among 3226 eligible patients, 1877 (58\%) had sleeve gastrectomy, 1136 (35\%) had Roux-en-Y gastric bypass, and $213(7 \%)$ had adjusted gastric banding. Most patients were female $(76 \%)$, and the mean age was 48 years. Median (interquartile range) preoperative BMI measured in the EHR was 44.8 (40.5-50.6) $\mathrm{kg} / \mathrm{m}^{2}$. Using claims-based features to predict preoperative BMI, algorithms capable of automatically performing variable selection and automatically modeling interactions and nonlinear relationships (random forest, XGBoost, and feedforward neural network) achieved an $R^{2}$ of $0.862-0.874$ and mean absolute error of 2.2-2.3 in the concurrent testing set. Other algorithms (linear regression, elastic net, and support vector machine) had lower but still decent performance, with an $R^{2}$ of 0.747-0.755 and mean absolute error of 3.1-3.3 in the concurrent testing set. Similar performance was observed in the prospective testing set.

Conclusion: In the absence of BMI measurements, claims-based models - especially models automatically implementing variable selection, interactions, and nonlinear relationships - can accurately predict preoperative BMI in bariatric surgery patients, thus facilitating confounding control by baseline severity of obesity in claims-based studies evaluating bariatric procedure outcomes. 


\section{A Natural Language Processing Approach to Improving the Use of Electronic Health Records for Identification of Patients With Social Risks and Needs}

Christopher Rouillard, Mahmoud Nasser, Haihong Hu, Kevin Rubenstein, Douglas Roblin

\section{University of California, Berkeley, Oakland, CA}

Background: Health systems are committed to addressing the impacts of social determinants of health $(\mathrm{SDoH})$ on patients' health and well-being. Advances in electronic health record (EHR) systems provide opportunities for systematic documentation and surveillance of historically undetected patient SDoH. Natural language processing (NLP) developments create a unique opportunity to generate a machine learning approach to identify patient SDoH.

Methods: We developed an open-source NLP approach to identify patient SDoH from unstructured free-text provider notes. This generalizable approach enables implementation across multiple EHR systems, geographic regions, and SDoH categories. Patient self-reported SDoH status (recorded by Your Current Life Situation [YCLS] survey) and associated provider notes recorded between March 2017 and June 2020 were extracted (32,261 beneficiaries; 50,722 YCLS surveys; 485,425 provider notes). Machine learning test statistic Term FrequencyInverse Document Frequency was used for NLP pattern generation. Patterns were developed and assessed in a training, training validation, and final validation dataset $(64 \%, 16 \%$, and $20 \%$ of total data, respectively). NLP models analyzed $3 \mathrm{SDoH}$ categories: housing instability and utilities payment (Housing), medical services payment (Medicine), and transportation assistance (Transportation). We also assessed model ability to identify a combined SDoH metric. Model performance was analyzed using sensitivity, specificity, and Cohen's kappa statistic, assuming the YCLS survey to be the gold standard.

Results: Within the training validation dataset, SDoHspecific NLP models showed strong sensitivity and specificity, with moderate agreement with the YCLS survey (Housing: sensitivity of 0.67 , specificity of 0.89 , Kappa of 0.51 ; Medicine: sensitivity of 0.55 , specificity of 0.73 , Kappa of 0.20 ; Transportation: sensitivity of 0.79 , specificity of 0.87 , Kappa of 0.58). Model performance in the training dataset was comparable to the training validation dataset. In the final validation dataset, a combined $\mathrm{SDoH}$ prediction metric showed sensitivity of 0.77 , specificity of 0.69 , and Kappa of 0.45 .

Conclusion: Our NLP algorithm demonstrated moderate performance in identification of overall SDoH status and specific unmet SDoH from digitized EHR provider notes compared to patient self-reports of SDoH. An NLP approach will enable improved surveillance of SDoH and, therefore, support delivery of interventions to patients unidentified by existing survey methods (nonrespondents, not in sample). NLP offers a novel approach for identifying unmet SDoH, improving resource allocation, monitoring SDoH goals, and ultimately improving patient health.
Development and Testing of a Risk-Standardized Inpatient Respiratory Depression Electronic Clinical Quality Measure for Orthopedic Practice

Ania Syrowatka, Avery Pullman, Woongki Kim, Stuart R. Lipsitz, Michael Sainlaire, Tien Thai, Wenyu Song, Troy Li, Alexandra Businger, David W. Bates, Patricia C. Dykes

\section{Brigham and Women's Hospital / Harvard Medical School, Boston, MA}

Background: Postoperative respiratory depression is a serious event that places patients at risk for hypoxia, anoxia, severe brain damage, cardiac arrest, and death. Key challenges to accurate and comparable measurement are lack of a universal definition, variability in measurement approaches, and heterogeneous populations. The objective of this work was to develop and test an electronic clinical quality measure (eCQM) that reports the inpatient respiratory depression rate following total hip arthroplasty and total knee arthroplasty.

Methods: Measure specifications were informed by a review of the literature, existing clinical quality measures as well as those under development, and consultations with a technical expert panel. Respiratory depression was measured using information routinely documented in electronic health records: respiratory depression-related diagnoses (eg, hypoxemia) or respiratory failure; mechanical ventilation or intubation procedure codes; and oxygen saturation (ie, $\mathrm{SpO}_{2}$ ) levels. Testing was conducted using data captured in the Mass General Brigham (MGB) electronic health record. The percentage of patients who experienced postoperative respiratory depression during the inpatient stay was calculated (unadjusted and riskstandardized), both overall and stratified by clinician group.

Results: At MGB, 16,474 patients received an inpatient elective primary total hip arthroplasty or total knee arthroplasty in 2016-2019 and were included for measure testing. Most patients were White (90\%), female (58\%), over the age of 65 years (58\%), and spoke English as their primary language (96\%). Overall, 545 patients experienced respiratory depression during the inpatient stay for an unadjusted rate of 3.3\%. Respiratory depression was identified based on documented diagnostic or procedure codes for 252 patients. An additional 293 patients $(\sim 50 \%$ of cases) were identified using documented oxygen saturation levels. There were 6 orthopedic clinician groups at MGB, and inpatient respiratory depression rates ranged from $2.5 \%$ to $6.7 \%$ (unadjusted). The overall risk-standardized inpatient respiratory depression rate at MGB was $2.7 \%$, with clinician-group rates ranging from $2.3 \%$ to $3.7 \%$.

Conclusion: Development of this eCQM addressed challenges associated with measurement of respiratory depression by leveraging data elements (specifically vital signs) routinely documented in electronic health records but not available in administrative health data. This approach allowed for more accurate estimates of inpatient respiratory depression and fair comparisons of performance between orthopedic clinician groups. 


\section{Use of Natural Language Processing to Ascertain Suicide Ideation/Attempt From Clinical Notes Within a Large Integrated Health System}

Fagen Xie, Deborah S. Ling Grant, John Chang, Britta I. Amundsen, Rulin C. Hechter

Kaiser Permanente Southern California, Pasadena, CA

Background: Identifying risk factors for suicide using clinical notes is time-consuming and usually requires manual case review. This study aims to develop and validate a natural language processing computerized algorithm to automatically ascertain suicide ideation/attempt from clinical notes in a large integrated health system, Kaiser Permanente Southern California (KPSC).

Methods: Clinical notes containing prespecified relevant keywords and phrases related to suicidal ideation/attempt between 2010 and 2018 were extracted from KPSC electronic health record system. A random sample of 864 clinical notes was selected and equally divided into 4 subsets. These subsets were reviewed and classified as 1 of 3 suicide ideation/attempt categories - "Current," "Historical," and "No" - for each note by experienced research chart abstractors. The first 3 subsets were used as training datasets to develop the computerized algorithm sequentially, and the fourth was used as a validation dataset to evaluate the algorithm performance. The validated algorithm was then applied to the entire study sample of clinical notes.

Results: The computerized algorithm ascertained 23 of the 26 confirmed Current suicide ideation/attempt events and all 10 confirmed Historical suicide ideation/attempt events in the validation dataset. This algorithm produced $88.5 \%$ sensitivity and $100.0 \%$ positive predictive value (PPV) for Current suicide ideation/attempt and $100.0 \%$ sensitivity and 100.0\% PPV for Historical suicide ideation/attempt. A total of 1,050,289 Current ideation/attempt events and 293,038 Historical ideation/attempt events were identified after the computerized algorithm was applied to the entire study population sample. Among the 400,436 individuals who were identified as having a Current suicide ideation/attempt event, 115,197 (28.8\%) were 15-24 years old at the first event during the study period, 234,924 (58.7\%) were female, 165,084 (41.7\%) were Hispanic, and 150,645 (37.6\%) had 2 or more events in the study period.

Conclusion: Our study showed that a natural language processing computerized algorithm can effectively ascertain suicide ideation/attempt from the free-text clinical notes in the electronic health record of a diverse patient population. This algorithm can be utilized in support of suicide prevention programs and patient care management.
Maximizing Use of Chronic Disease Clinical Decision Support in Primary Care Clinics: Lessons Learned From Randomized Trials

JoAnn Sperl-Hillen, Heidi L. Ekstrom, Kris A. Ohnsorg, Daniel Saman, Stephen Waring, Thomas Elliott, Clayton Allen, Deepika Appana, Patrick J. O'Connor

HealthPartners, Bloomington, $M N$

Background: High and sustained clinical decision support (CDS) use rates are a necessary precondition for CDS success, because CDS systems that are not used are unlikely to improve care. In this comparative case report, we describe various strategies used for CDS training, financial incentives, feedback of CDS use rates, and other factors associated with both high and low CDS use rates in primary care clinics.

Methods: Comparative case report of 3 National Institutes of Health-funded CDS implementation projects that address cardiovascular risk factor control in primary care settings. These studies were conducted in 3 large multispecialty medical groups over a 10-year period of time.

Results: In Project A, CDS use rates rose to $60 \%$ at targeted primary care encounters over a 6-month period of time using in-clinic training and financial incentives to clinicians and office staff. In Project B, CDS use rates rose to $70 \%$ using in-clinic training, feedback of CDS use rates (at targeted encounters) to clinic leaders and individual clinicians, but with no financial incentives. In Project C, CDS use rates rose to $50 \%-60 \%$ after virtual CDS training, with feedback of CDS use rates and no financial incentives, subsequently fell when the proportion of targeted encounters was increased, but rose again after face-to-face in-clinic refresher training. For CDS aimed only at diabetes patients, clinicians could accurately identify target patients, but when targeting was based on less obvious measures of cardiovascular risk, algorithmic identification of targeted patients was required.

Conclusion: These case studies suggest that training delivered using existing local medical group training procedures or customs is less effective than face-to-face training in clinic settings. We found little to support the use of financial incentives to providers as an effective strategy to achieve or maintain high CDS use rates, although incentives to clinic "pools" was effective. We found strong evidence that monitoring and feedback of primary care provider-specific and clinic-specific CDS use rates by name is an effective strategy to initiate and sustain desired levels of CDS use in primary care clinics. 


\section{EPIDEMIOLOGY AND SURVEILLANCE}

\section{Review of Factors Associated With Number Needed to Screen for Revised World Health Organization Principles and Recommendations for Systemic Screening of Active Tuberculosis}

\section{Rabia Jalalzai \\ Johns Hopkins School of Public Health, Baltimore, MD}

Background: As part of the larger systematic review of number needed to screen (NNS) to detect 1 case of active tuberculosis (TB) per the revised World Health Organization (WHO) principles and recommendations for systematic screening of active TB, this paper's main objective is to determine factors that affected NNS to detect a single case of TB among 21 studies emerging from WHO's Eastern Mediterranean Region.

Methods: We first calculated TB prevalence by dividing the number of confirmed active TB cases by the total number of people screened in each study. NNS counts to find 1 case of active TB were then calculated as the inverse of TB prevalence. Results were obtained using Stata ${ }^{\circledR}$ software.

Results: The overall NNS count to detect 1 case of active TB was 98. Within screening strategies, screening based on TB symptoms yielded NNS of 186 , chest X-ray 38 , and sputum smear 21. Among the diagnosis strategies, culture as a method of TB ascertainment had NNS of 200 and Xpert yielded NNS of 42.

Conclusion: NNS calculations vary depending on screening strategies, diagnostic methods, and the risk groups included in each study but can help guide decisions when planning and carrying out active case finding. Among the screening strategies, chest X-ray and smear may be high yield with comparatively smaller NNS than symptom screening. Among diagnostic tactics, Xpert resulted in a much smaller mean NNS compared to culture, suggesting that it may be valuable to include Xpert as a way of TB confirmation when planning for active case finding. Studies that screened children had a much smaller mean NNS compared to those that screened adults, indicating the need to set a higher NNS threshold when screening adults. Clinical diagnosis for populations of children yielded NNS of 32, proposing that it may be worthwhile to include clinical diagnosis as an active TB confirmation method for this age group. Within the risk groups, persons with diabetes and HIV resulted in much smaller NNS compared to those with opioid drug use disorder. An arbitrary threshold of the number needed to screen set at 105 or above will result in all 3 risk groups included as a priority group for active case finding.

\section{Emergency Room Utilization and Hospital Admissions Among Patients With Monoclonal Gammopathy of Undetermined Significance}

Maira A. Castaneda Avila, Kate Lapane, Sharina Person, Yanhua Zhou, Bill Jesdale, Kathleen M. Mazor, Mara Epstein

University of Massachusetts Medical School, Worcester, MA

Background: Monoclonal gammopathy of undetermined significance (MGUS) is an understudied premalignant disorder. Patients with MGUS progress to multiple myeloma at a rate of $1 \%$ per year and undergo regular clinical surveillance with associated anxiety. We examined differences in emergency department visits (ER) and hospital admissions in patients with and without MGUS to evaluate the impact of receiving an MGUS diagnosis on health care utilization.

Methods: This matched cohort study included 429 patients with MGUS diagnosed in central Massachusetts from 2007 to 2015 and 1286 patients without MGUS matched on sex, age ( \pm 2 years) and length of enrollment in the health system. Frequency of ER visits and hospital admissions were assessed in 3 time frames: 1) 1 year before diagnosis/ index date; 2) 1 month before and after diagnosis/index date; and 3) 1 year after diagnosis/index date. Multivariable conditional Poisson models were used to evaluate the magnitude of change in health care utilization in patients with MGUS as compared to patients without MGUS.

Results: Half the population was female, with a mean age at diagnosis/index date of 75 years. The majority was nonHispanic White (MGUS: $85 \%$ vs non-MGUS: $81 \%$ ), and the mean Charlson comorbidity index score was higher for patients with MGUS (2.3) than those without (1.6). The rate of ER visits was $73 \%$ (95\% CI: $1.1-2.8$ ) higher for those with MGUS than patients without MGUS in the month before and after diagnosis/index date and 51\% higher for the year after diagnosis (95\% CI: 1.25-1.82). The rate of hospital admissions was 4 times higher $(95 \%$ CI: 2.8-7.8) for those with MGUS than for patients without MGUS in the period around diagnosis. The association was attenuated, although still elevated, in the year following diagnosis (incidence rate ratio: 1.67, 95\% CI: 1.4-2.00).

Conclusion: Our study suggests that MGUS diagnosis is associated with a higher increased utilization of ER visits and hospitalizations, especially during the month surrounding the diagnosis date. Further studies are warranted to examine the reason behind this increased utilization. 


\section{A Spatial Analysis of Mental Health Disorder and Crime Rates in St. Louis, Missouri}

\section{Ness Sandoval, Ruaa Al Juboori, Shreya Nagendra}

Saint Louis University / SSM Health, St. Louis, MO

Background: Urban mental health remains an important dimension in the study of neighborhood inequality. Yet, few studies have examined the relationship between neighborhood mental health and crime.

Methods: The 4 data sources used in this study were American Community Survey 2018 5-Year, PolicyMap database, St. Louis Metropolitan Police, and TIGER shapefiles from the U.S. Census. Using a geospatial methodological framework, this study explored the spatial relationship of neighborhood mental health disorder and crime rates for 106 census tracts in St. Louis. Missouri. This study operationalized mental health disorder using 3 variables: 1) self-report depression; 2) selfreport of days of mental distress disorder; and 3) a depression index. Analyses were done using ArcGis and GeoDa.

Results: The Moran's I analysis confirmed that all 3 mental health disorder measures had spatial autocorrelation and that they were all significant. Self-report depression had the highest Moran's I value. The Moran's I analysis also showed that neighborhood crime rate had moderate but statistically significant spatial autocorrelation. The spatial autoregressive model (SAR) showed conflicting results. The SAR model found no evidence that neighborhood crime rates were associated with days of mental distress. However, the SAR model did find weak evidence that neighborhood self-report depression and the depression index were associated with crime rates; this was an unexpected direction (ie, associations opposite to our expectations).

Conclusion: While our results overall suggest that crime minimally influenced depression, additional research is needed, including to understand results in the unexpected direction.

\section{Accuracy of Tumor Registry Data for Capturing Endocrine Therapy Treatment Compared to Pharmacy Data Among Women With Breast Cancer From an Integrated Health Care Delivery System}

Cameron Haas, Erin Bowles, Janie Lee, Jennifer Specht, Diana Buist

\section{Kaiser Permanente Washington, Seattle, WA}

Background: Accurately accounting for adjuvant breast cancer treatment is important for studies evaluating surveillance effectiveness and second events following initial treatment. Previous studies have documented incomplete endocrine therapy ascertainment in the population-based Surveillance, Epidemiology, and End Results (SEER) registry based on Medicare claims data for breast cancer. We evaluated the accuracy of SEER treatment data for endocrine therapy in women with breast cancer using pharmacy data from our Virtual Data Warehouse.

Methods: We included 6925 women with a primary breast cancer diagnosis during 1995-2017 enrolled in Kaiser Permanente Washington. We used pharmacy data as our gold standard and included any prescription dispensings for aromatase inhibitors, selective estrogen-receptor (ER) modulators, and GnRH agonists following diagnosis dates. We calculated kappa (concordance), positive predictive value (PPV), and negative predictive value (NPV) overall and within patient and tumor characteristics of interest. We calculated time to first prescription dispensing as the difference in days between diagnosis and date of first fill in our claims data.

Results: Our cohort had a median age of 62 years; $84 \%$ were ER+/progesterone receptor (PR)+, 45\% localized stage, just over half received lumpectomy with radiation (54\%), and only $29 \%$ received chemotherapy. Among the 5098 women with ER+/PR+ breast cancer, SEER data identified $67 \%$ as having received endocrine therapy. This increased to $77 \%$ when we added pharmacy dispensing data. In the 566 cases for which receipt of endocrine therapy was not recorded by SEER, most (76\%) had their first fill within 12 months of diagnosis whereas $15 \%$ had their first fill more than 2 years postdiagnosis. SEER's concordance with pharmacy dispensings was 0.76 , with PPV of $95 \%$ and NPV of $81 \%$. PPV did not vary across most patient and tumor characteristics; however, NPV declined with younger age at diagnosis ( $75 \%$ for age of $<45$ years vs $87 \%$ for age of $75+$ years), increasing tumor stage ( $71 \%$ for regional stage vs $93 \%$ for in situ), and chemotherapy treatment $(72 \%$ for those with chemotherapy vs $84 \%$ for those without chemotherapy). Conclusion: Claims and pharmacy data enable more complete capture of endocrine therapy data, increasing accuracy of treatment data compared with SEER data alone.

\section{GENOMICS AND PRECISION MEDICINE}

\section{Prospects of the Global Precision Medicine Market}

Muge Yang, Bin Li

Washington Institute for Health Sciences, Arlington, VA

Background: With the rapid development of next-generation gene sequencing technology and information technology, the medical model is changing from the traditional "onesize-fits-all" model to the precision medical model, which is based on the specificity of individual genetics, environment, and lifestyle to develop individualized treatment and prevention plans.

Methods: We used "precision medicine market"' as the 
keyword to search the literature on ProQuest Central and downloaded the 200 most relevant articles. Based on the data in the literature, we estimated the value of the current global precision medicine market, including ecosystems, end users, therapies, and geographic regions, as well as the global market value in the next 10 years, in U.S. dollars.

Results: The factors that promote the development of precision medicine include technological progress, market demand, changes in medical models, public attention, and government action. The factors hindering the development of precision medicine include high price, technical limitations, scarcity of professionals, and lack of appropriate policies and regulations. The global precision medicine market size is estimated at $\$ 53.7$ billion- $\$ 87.2$ billion in 2019 , and it is expected to reach $\$ 146.8$ billion- $\$ 278.6$ billion by 2030 . The compound annual growth rate from 2020 to 2030 is $11.13 \%$. The precision medicine market can be segmented by ecosystem, end user, treatment, and geography. By ecosystem and end user, diagnostic-related segments occupy the main part of the precision medicine market. By therapeutics, the oncology segment dominates the precision medicine market with an estimated market share of more than $30 \%$. North America is the largest and most potential regional precision medicine market; in 2019, its market was $\$ 31.4$ billion- $\$ 54.6$ billion and contributed approximately $35.0 \%-46.8 \%$ of the global market values. Europe is the second-largest market with a market size of $\$ 15.1$ billion-\$34.3 billion in 2019 . The Asia-Pacific region is the fastest-growing precision medicine market with a compound annual growth rate of $14.4 \%-16.6 \%$ in the next few years. It is foreseeable that precision medicine will be one of the fastest-growing industries in the world over the next 10 years.

Conclusion: It is foreseeable that precision medicine will be one of the fastest-growing industries in the world over the next 10 years, and the growth rate will remain over $10 \%$.

\section{Genetic Risk Factors for Suicidal Behaviors Among Community-Based Veterans: Exploratory Results From the Veterans' Health Study}

Joseph A. Boscarino, Richard E. Adams, Joseph J. Boscarino, Thomas G. Urosevich, Stuart N. Hoffman, H. Lester Kirchner, Ryan J. Dugan, Carrie A. Withey, Charles R. Figley

\section{Geisinger Research, Danville, PA}

Background: Since suicide among veterans is a concern among health care providers and our genetic knowledge of this outcome is still limited, our objective was to assess genetic risk factors for suicide among community-based veterans. We studied a random sample of veterans who were outpatients in a non-Veterans Affairs (VA), large multihospital system in central/northeastern Pennsylvania.
Methods: Using diagnostic surveys, we interviewed 1072 veterans by phone, $62.3 \%$ of which were deployed to Vietnam and $37.7 \%$ deployed to post-Vietnam War conflicts. The interviews took $\sim 65$ minutes to complete and collected data on suicide, posttraumatic streess disorder (PTSD), depression, combat and trauma exposures, and demographic and psychosocial factors. DNA was collected via Oragene saliva kits (DNA Genotek Inc.), which were returned by mail following the survey. The genetic risk variants studied were within loci previously associated with PTSD, including CRHR1, CHRNA5, RORA, and FKBP5 genetic variants, which were used to calculate a polygenic risk score (range: 0-8, mean: 3.6, standard deviation [SD]: 1.4) (https:// pubmed.ncbi.nlm.nih.gov/32021198/). Non-Whites $(n=43)$ were excluded from these analyses.

Results: Among those who ever attempted suicide or had a suicide plan, the PTSD genetic risk score was significantly higher (3.96 vs 3.55; $\mathrm{F}=4.54 ; \mathrm{P}=0.033$ ). The mean number of lifetime trauma exposures among veterans was 3.5 (SD: 2.1). In multivariable logistic regressions, significant predictors of attempting suicide or having a suicide plan were history of depression (odds ratio [OR]: 7.4; $\mathrm{P}<0.001$ ), PTSD risk score (OR: $1.3 ; \mathrm{P}=0.017$ ), and lifetime trauma exposures (OR: 1.2; $\mathrm{P}=0.010$ ). Significant predictors for developing a suicide plan were history of depression (OR: 8.8; $\mathrm{P}<0.001$ ), PTSD risk score (OR: 1.2; $\mathrm{P}=0.045$ ), lifetime trauma exposures (OR: $1.2 ; \mathrm{P}=0.035$ ), and serving on multiple combat tours (OR: 0.49; $\mathrm{P}=0.038$ ).

Conclusion: In this study, we identified novel genetic risk variants for suicidal behaviors among veterans that may have implications for future genomics and precision medicine. These exploratory findings may be relevant for health care providers and others working in both VA and non-VA facilities in the future. Further research is advised.

The Kaiser Permanente Northwest Biorepository: A Valuable Resource for Collaborative Research

Joanna Bulkley, Jessica Hunter, Allison Naleway, Shawn Westaway, Yolanda Prado, Alan Bauck, Sarah Vertrees, Charisma Jenkins, Sheila Weinmann

\section{Kaiser Permanente Northwest, Portland, OR}

Background: The Center for Health Research (CHR) at Kaiser Permanente Northwest (KPNW) provides researchers access to a unique collection of blood and tissue specimens for use in research studies in collaboration with CHR investigators. Specimens are linked to each patient's electronic health record, which documents the full scope of our members' medical care, including data from encounters, pharmacy, and laboratory testing. Survey and/or genomic data are available for some participants and tumor registry data for members with cancer. Specimens from participants who now have COVID-19 test results also are available. 
Methods: Specimens were gathered for research studies and by retaining specimens after usual clinical care. Bloodderived specimens include plasma, DNA extracted from whole blood, and buffy coat that can be used to obtain genomic data. Tissue specimens include individual formalinfixed paraffin-embedded (FFPE) normal and tumor tissue blocks dating back to 1971 and associated hematoxylin and eosin (H\&E)-stained slides.

Results: The KPNW biorepository contains over 60,000 blood-derived specimens from more than 40,000 individuals. The majority are female $(59 \%),<70$ years old $(57 \%)$, and non-Hispanic White (89\%). Large disease cohorts include diabetes $(>5000)$, cardiovascular disease $(>3000)$, cancer $(>2800$ breast, prostate, and lung), and clinical depression (>1300). Specimens from participants with other diseases, including hypertension, hemochromatosis, hip fracture, and cervical dysplasia, are available, as are specimens from volunteers without a specific disease $(>2000)$. There are more than 3.5 million normal and tumor tissue blocks and H\&E slides. Of the more than 95,000 tumors in the library, tissue is available from more than 15,000 breast, 10,000 prostate, 7500 cervical, 7500 colon and rectum, 6000 lung, and 6000 urinary system cancer patients.

Conclusion: Linking KPNW's clinical data with DNA and other biological specimens creates a powerful research resource. Our specimens can be used for a wide range of studies: studies of molecular and genetic factors associated with disease incidence and progression; pharmacogenetic studies; and disease detection, prevention, and treatment studies. To date, our biospecimens have been used to predict response to tamoxifen in breast cancer treatment, evaluate the pharmacogenetics of CYP2C19 in proton pump inhibitor use, and identify genetic risk factors for aggressive prostate cancer.

\section{HEALTH CARE DELIVERY AND COVERAGE}

\section{A Mixed-Methods Evaluation of Medication Reconciliation Using Interviews and Surveys in Primary Care Practices}

Michael Gionfriddo, Vanessa Duboski, Jove Graham, Melissa Kern, Eric Wright

\section{Geisinger Research, Forty Fort, PA}

Background: Failure to conduct proper medication reconciliation results in inaccurate medication lists, leading to avoidable health care utilization and costs. To better understand the factors that contribute to inaccurate medication lists, we conducted a mixed-methods evaluation as part of a quality improvement project to improve medication reconciliation in our primary care practices.

Methods: We conducted a mixed-methods study consisting of semi-structured interviews and surveys of both patients and staff within ambulatory care at a large integrated health care delivery system. Interviews were conducted telephonically throughout 2019 and 2020 using semistructured interview guides informed by findings from clinic observations and peer-reviewed literature. Interviews were recorded and analyzed thematically using a codebook and Atlas.ti 8. Surveys, developed based on clinic observations, staff interviews, and existing literature, were administered electronically utilizing REDCap during the summer of 2020. Surveys were analyzed using descriptive statistics completed in SAS and R software.

Results: We conducted 48 staff and 10 patient semi-structured interviews. Surveys were sent to 2541 eligible staff, of which 616 responded (24\% response rate), and 5132 eligible patients, of which 577 responded (11\% response rate). Both patients and staff felt having an accurate medication list was important (96\%); however, several factors influenced the collection of a patient's medication list. These factors included the lack of a standardized process (and subsequently a lack of clarity around responsibility for and comfort with the process), a lower level of concern for nonprescription medications, a lack of awareness and comfort with available tools to facilitate medication reconciliation, a lack of time, inadequate staff training for conducting proper medication reconciliation, poor communication within and across sites of care, and a lack of knowledge of medications by both patients and staff.

Conclusion: Using a thorough mixed-methods evaluation of the process involved in collecting an accurate medication list in ambulatory care, we have identified several barriers impacting the process of medication reconciliation. Future work has the potential to reduce medication list discrepancies, improve outcomes, and reduce avoidable health care utilization costs.

Factors Associated With Preferences for Continued Video Visit Use During the COVID-19 Pandemic

Xiaowei (Sherry) Yan, Sien Deng, Su-Ying Liang, Cheryl Stults, J.B. Jones, Ellis C. Dillion, Dominick Frosch

\section{Sutter Health, Walnut Creek, CA}

Background: Use of video visits (VV) skyrocketed during the first wave of the COVID-19 pandemic, as in-person visits were canceled to minimize exposure risk. Little is known about which VV users are most likely to prefer a VV in the future and why. We combined a population-based survey with electronic health records to identify patient characteristics and other factors affecting VV preferences.

Methods: In August 2020, we invited a stratified random sample of 20,000 active patients at Sutter Health to complete an email survey on patient health care-seeking preferences 
and VV experiences during COVID-19. We combined survey and electronic health record data and applied a weighted logistic regression model, adjusting for sampling frame and response bias, to identify patient characteristics, clinical factors, VV experience, and other factors associated with preferences for future VV use.

Results: A total of 3351 patients completed the survey (18\% response rate), among which 1208 (37.4\%) reported having at least one VV. Among 5 dimensions for assessing VV experience, $49 \%$ were very satisfied with the length of time with the doctor, and $40 \%$ were very happy using VV to talk to their doctor. Fewer patients $(\sim 36 \%)$ reported being "very happy" with the overall quality of the visit or scheduling experience. Among 1208 patients who reported having VV before, 38\% $(n=460)$ would prefer a VV in the future. Satisfaction with using a VV to interact with their doctor and overall VV quality were the strongest factors associated with a preference for a future $\mathrm{VV}$ (odds ratio [OR]: 5.30, 95\% CI: 3.57-7.85 and OR: 3.94, 95\% CI: 2.66-5.86, respectively). Hispanic ethnicity (OR: 0.52, 95\% CI: 0.30-0.92, compared to White race) and older (65-79 and 80+ years) age (OR ranges: $0.26-0.50$, compared to $18-49$ years of age) were less likely to prefer a future VV.

Conclusion: More than one-third of surveyed patients participated in a VV during the COVID-19 pandemic, and patients' perception of overall VV quality is significantly associated with preference to use VVs in the future. As COVID changes health care delivery, it is critical to understand who, when, and how to deliver telemedicine options that meet patient's needs and to optimize the value of telemedicine in the overall care delivery landscape.

\section{Replacement of Electrolyte in the Intensive Care Unit: Study of Pattern in MIMICS III Database}

Parasteh Malihi, Mousa Ghannam, Krzysztof Laudanski

\section{University of Pennsylvania, Philadelphia, PA}

Background: Electrolyte repletion is a ubiquitous routine intervention aimed at maintaining the homeostatic range in serum. However, variations in the replacement practices and belief systems surrounding the clinical effectiveness of electrolyte repletion may be resulting in substandard care. While low electrolyte levels are associated with poor outcomes, higher levels are not always considered protective - even more, they can be harmful. Current patterns of electrolyte repletion in the intensive care unit (ICU) suggest overrepletion, with significant percentage being dosed when the levels are therapeutic.

Methods: Herein, we analyzed ICU electrolyte replacement patterns using the MIMICS III database to determine the threshold governing replacement decisions and their efficiency. We evaluated the data for serum values for potassium magnesium, calcium, and phosphate before and after repletion events. We identified the thresholds for when repletion was administered for the mentioned electrolytes. Additionally, we were able to identify temporal patterns in repletion behaviors of ICU health care providers.

Results: While a majority of replacements for potassium, magnesium, calcium, and phosphate occurred at levels below or within reference ranges, the percentage of repletion events that fell above the reference ranges for potassium, calcium, and phosphate was $0.79 \%, 0.34 \%$, and $0.46 \%$, respectively. Interestingly, occurrence of replacements for magnesium above reference ranges was $3.37 \%$ of the events. The electrolyte repletion resulted in mainly small to modest postreplacement changes in electrolytes serum level. Repletion behavior patterns were associated with hospital workflow more so than with clinical indication.

Conclusion: This pattern of behavior found in this study supports a wasteful and ineffective electrolyte replacement practice in a different medical system, pointing to the universality of the problem.

\section{Demographic and Clinical Differences in Achieving Guideline-Recommended Cardiometabolic Screening for Youth Prescribed Antipsychotic Medications}

\author{
Loretta Hsueh, Natalie Slama, Scott Spalding, Stacy \\ Sterling, Esti Iturralde
}

\section{Kaiser Permanente Northern California, Oakland, CA}

Background: Antipsychotic medications are used to treat various mental health conditions in youth but are associated with metabolic disturbances that elevate cardiovascular health risks and guideline-recommended cardiometabolic screening of these youth remains low. We examined demographic and clinical predictors of cardiometabolic screening for this population in a large integrated health system to inform understanding of screening facilitators and barriers.

Methods: In this serial cross-sectional study, we assessed cardiometabolic screening (glucose or lipid test) from health record data for 4568 youth (10-21 years of age) starting antipsychotic treatment in Kaiser Permanente Northern California during 2013-2017. Using logistic regression, we assessed demographic and clinical predictors of cardiometabolic screening within 2 years of medication start. We examined whether distinct characteristics predicted screening for boys versus girls using gender-stratified models, confirming statistical significance of differences via gender interaction terms added to the original model. Below, we report adjusted metabolic screening frequencies generated from model coefficients.

Results: Participants were of mean age 17 (standard deviation: 3.0 ) years; $52 \%$ were boys. Half were racial/ ethnic minorities (22\% Hispanic/Latino; 13\% Black; 12\% 
Asian/Nat. Hawaiian/Pac. Islander [ANHPI]). Almost 1 in 5 youth $(18 \%)$ had age-adjusted obesity at baseline. On average, $54 \%$ of youth were screened within 2 years of starting an antipsychotic. Younger participants, girls, Hispanic/Latino participants, and those with a diagnosis of attention-deficit/hyperactivity disorder, bipolar disorder, or psychotic disorder had higher screening rates, whereas participants with a diagnosis of substance use disorder had lower screening rates overall $(\mathrm{P}<0.05$ for all). Adjusting for mental health and primary care utilization had negligible effects on these associations, although girls were no longer more likely than boys to be screened after adjustment. Girls with obesity were more likely to be screened than boys with obesity (63\% vs 53\%). ANHPI race/ethnicity predicted higher screening only in boys $(61 \%)$ relative to White race/ ethnicity (51\%).

Conclusion: In this diverse sample of youth treated with antipsychotic medications, a marker of cardiometabolic health risk (obesity) predicted glucose or lipid screening only in girls. Screening prevalence was especially high among ANHPI boys. Future research should examine processes contributing to these differences to inform strategies seeking to increase health-protective screening.

\section{Association Between Palliative Care Consultation, Hospital Length of Stay, and In-Hospital Charges Among Women With Metastatic Breast Cancer in the United States}

Divya Subramaniam, Noor Al-Hammadi, Alexandria Jenkins, Leslie Hinyard

\section{Saint Louis University / SSM Health, St. Louis, MO}

Background: There is limited research about the utilization of palliative care services for women with metastatic breast cancer, particularly at the end of life. The purpose of this study was to determine the incidence of inpatient palliative care consultation (PCC) in women with metastatic breast cancer and to examine the association between PCC, hospital length of stay, and in-hospital charges in a nationally representative sample.

Methods: Women with a metastatic breast cancer-related hospitalization in the Healthcare Cost and Utilization Project Nationwide Inpatient Database (NIS) during 19982017 were included in the study. Multivariable logistic and linear regression analyses were used to examine the effects of patient and hospital characteristics on the receipt of inpatient PCC, length of stay, and total hospital charges. SAS software was used for all analyses.

Results: Average age of the patient sample $(\mathrm{N}=513,509)$ was 63 years; $73.2 \%$ of women were White, received governmental types of insurance. Older women (60-69 years) had higher odds of getting PCC. Women of races other than White, and patients from zip codes with lower household income levels were more likely to receive PCC. This finding was evident among women who received other types of insurance as compared to private insurance. Women receiving governmental insurance had slightly lower odds of receiving palliative care compared to women receiving commercial types of insurance. Small and medium-sized hospitals were less likely to offer the service to admitted women. Rural and urban nonteaching hospitals were associated with lower odds of providing PCC to their admitted patients. A woman with comorbidity had higher odds of receiving PCC during their hospital admission. Hospitals were likely to charge patients receiving palliative care services less compared to patients who did not have a PCC encounter despite being associated with higher odds of a longer hospital stay.

Conclusion: We found that inpatient PCC for women with metastatic breast cancer increased hospital length of stay and reduced hospital total charges. We also found racial differences in inpatient palliative care consultation, with it being restricted to older and sicker patients. Targeted interventions must be implemented to increase PCC for patients with metastatic breast cancer in the United States.

\section{Palliative Care Among Patients With Metastatic Breast Cancer in the End-of-Life Period in the United States}

\section{Divya Subramaniam, Zidong Zhang, Alexandria Jenkins, Leslie Hinyard}

\section{Saint Louis University / SSM Health, St. Louis, MO}

Background: Palliative care improves the quality of an individual's end of life. The purpose of this study was to determine utilization of palliative care consult (PCC) on or after date of metastasis to date of death and the association of emergency department (ED), intensive care unit (ICU), and chemotherapies among patients with metastatic breast cancer in the United States.

Methods: We conducted a retrospective study using the de-identified electronic medical record data from a national health care informatics provider and insurer from January 1, 2000 , to December 31,2018 . Adult patients ( $\geq 18$ years old) with breast cancer who were diagnosed with a secondary neoplasm after the index diagnosis of breast cancer and deceased in the period of data capture were included. The cohort was split into a PCC group — patients who had received at least one $\mathrm{PCC}$ on or after the date of metastasis through date of death - and a non-PCC group consisting of patients who not did not receive any PCC. The ED, ICU, and chemotherapy encounters since metastasis were defined by ICD-9/10, HCPCS or CPT codes per encounter records. We quantified the ED and ICU visits within 2 months of death and the antineoplastic chemotherapies within 1 and 2 months of death. Time from metastasis to death and 
utilization of services were compared between the PCC and non-PCC groups using Whitney U-test and chi-squared test. Results: Overall, 979 deceased patients with metastatic breast cancer had at least one PCC. Average patient age at the last admission was 68 years. Median time from metastasis to death was 9 years. Most were Caucasian (79.8\%) and from the U.S. Midwest (47.1\%). PCC patients had a shorter time to death and were more likely to be African American, reside in the Midwest, and have experienced complications since metastasis (except for acute cerebrovascular events). PCC patients were more likely to have used the ED or ICU within 2 months of death and to receive chemotherapy within 1 and 2 months of death.

Conclusion: Only $38 \%$ of patients with breast cancer received at least one PCC following date of metastatic diagnosis. Our findings highlight the need to increase PCC among patients with breast cancer immediately following metastasis diagnosis to ensure appropriate care is provided and utilized.

\section{HEALTH EQUITY AND SOCIAL NEEDS}

\section{Virtual Health Equity Tours as an Education Tool in Health Care Institutions}

Jaqui Melton

\section{BJC HealthCare, St. Louis, MO}

Background: Prior to the onset of the COVID-19 pandemic, our health care institution engaged in community tours that explored the social determinants of health $(\mathrm{SDoH})$ within the context of St. Louis health disparities, history, and geography. Further, it offered an opportunity for participants to better understand historical mistrust that members of marginalized communities hold when it comes to expectations of care in institutional spaces. While the experience was hailed as a staple diversity, equity, and inclusion effort, two paramount limitations were that the tour was limited to leadership participation and that it could only be experienced by up to 8 people at a time.

Methods: Amid COVID-19, we were provided the opportunity to reimagine the tour. In doing so, we explored what it would look like to expand capacity and accessibility. Further, we investigated and tested a variety of mediums including videos and interactive map technology — to create an experience that fosters and leverages community voice, activates and draws on participant wisdom, and provides a relevant background and framework to help inform how we move forward in caring for communities placed most at risk. Results: To date, a virtual health equity tour (VHET) has been piloted with 5 different groups comprised of team members from a variety of different ranks and roles within our organization. The feedback has been overwhelmingly positive. Among the quantitative results, $98 \%$ of participants said they would recommend the tour to a colleague and $100 \%$ of participants ranked the tour "very good" or "excellent." Qualitative findings included, but were not limited to, relevance and applicability to patient and team member experiences, the necessity of using history to inform how we make equitable decisions in the present and future, and making the tour an onboarding standard for all employees.

Conclusion: The VHET has provided an increasingly accessible way for providers to interactively learn about $\mathrm{SDoH}$ within the context of communal history. By the end of the tour, many participants were inspired to action, with some stating: "This tour motivated me to learn more about the history of St. Louis and continue to advocate;" and "It lit a fire inside of me to do more for our communities."

\section{Patient Characteristics Associated With Telemedicine Choice During the COVID-19 Pandemic Among Patients With Limited English Proficiency}

Loretta Hsueh, Jie Huang, Anjali Gopalan, Mary Reed

Kaiser Permanente Northern California, Oakland, CA

Background: Dramatic increases in telemedicine during the COVID-19 pandemic have made addressing disparities in telemedicine use a high priority. Patients with limited English proficiency (LEP) face significant barriers to care and to telemedicine, particularly video-based telemedicine. Understanding patient characteristics associated with telemedicine choice (video vs phone) can inform interventions to reduce disparities in video telemedicine use. Methods: Cross-sectional analysis of 22,476 primary care telemedicine visits initiated by patients with LEP scheduled through the Kaiser Permanente Northern California patient portal from March 16, 2020, to October 31, 2020. Using multivariable logistic regression, we identified correlates of choosing video vs phone visit, including patient language, race/ethnicity, gender, age, neighborhood-level socioeconomic status (SES), technology access (mobile portal use in past 12 months, neighborhood-level internet), past video visit experience (in past 12 months), and visit provider (eg, patient's own primary care provider).

Results: Video visits accounted for $34.6 \%(n=7,765)$ of all telemedicine visits initiated by patients with LEP; the most common languages spoken included Spanish (42\%), Cantonese (16.8\%), Mandarin (10.3\%), Vietnamese $(8 \%)$, Punjabi (3.3\%), and Russian (2.3\%). Compared to Spanishspeaking patients, Mandarin-speaking patients had higher odds of choosing video vs phone (odds ratio [OR]: 1.34) whereas Punjabi-speaking patients had lower odds (OR: 0.66). In addition, odds of choosing video were higher among adults younger than 18 years old (OR: 1.67) or older than 65 (OR: 1.24), those who previously accessed the 
mobile portal (OR: 1.17), and those with prior video visits (OR: 2.37). Odds of choosing video were lower among patients who lived in a low-SES neighborhood (OR: 0.89) or lived in a neighborhood with low internet connectivity (OR: $0.83 ; \mathrm{P}<0.05$ for all).

Conclusion: During the ongoing COVID-19 pandemic, the majority of telemedicine visits scheduled by adults with LEP were conducted via telephone. Telemedicine choice differed by specific Asian languages, underscoring the need to disaggregate data by Asian ethnic groups. Technology access factors, such as internet connectivity, may remain substantial barriers to video telemedicine use. In contrast to prepandemic studies in general patient populations, adults $65+$ years old with LEP had higher odds of video telemedicine use compared to adults $18-64$ years old. Future research should investigate mechanisms leading to higher video use among older patients with LEP, such as the role of family support in using video telemedicine.

\section{Identifying Social Needs Among Patients Referred to an Opioid-Tapering Program in a Large Integrated Health Care Delivery System}

\author{
Dea Papajorgji-Taylor, Phillip Crawford, Katherine \\ Reese, Mary Ann McBurnie
}

\section{Kaiser Permanente Northwest, Portland, OR}

Background: Due to a significant number of patients receiving high-dose opioid prescriptions, an integrated health system implemented a phone-based, pharmacistled program to support primary care physicians and assist patients in tapering. Prevalence of screening patients for unmet basic needs among this population has not been explored, and much less is known about how pharmacists view the role of identified social needs into their opioidtapering plans.

Methods: We reviewed electronic health records of patients referred to the tapering program and either were screened for socioeconomic needs, had a community resource referral placed on their behalf, or both, January 1, 2017-December 31, 2019. Referrals were identified by the community referral code in the electronic health records. Descriptive analysis was applied to identify associations among patient characteristics, unmet basic needs, and frequency of referrals via clinical screening. Qualitative data from the pharmacists facilitating the opioid tapers was collected and analyzed regarding the role that screening for socioeconomic needs can play among patients referred to the opioid-tapering program.

Results: Initial findings indicate 3369 patients were referred to the tapering program during the time frame examined. A total of 870 patients referred completed the Your Current Life Situation questionnaire, and 330 identified at least 1 unmet need. Community resource referrals were made for
427 patients. A total of 1222 referrals (multiple referrals per patient) identified transportation as the most prevalent need $(21.9 \%)$, followed by government assistance programs $(14.3 \%)$, and housing and shelter (11.4\%). Qualitative data from the pharmacists emphasized the value placed on patient-reported unmet needs when formulating a taper plan. Participants noted that various socioeconomic unmet needs have played a role in their patients' abilities to successfully adhere to a developed tapering plan or pain management.

Conclusion: Findings suggest unmet basic needs and community referrals are frequent among insured patients referred to an opioid-tapering program. We will describe associations between social needs status and patient demographics as well as the role of social needs screening among pharmacists facilitating patient tapers. Future research should consider identifying and addressing unmet socioeconomic needs among patients participating in the opioid-tapering program and develop a tapering plan that incorporates underlying social determinants of health.

\section{Development and Validity of 1- and 2-Item Screeners for Identifying Social Needs in a Health Care Setting}

Alicia L. Salvatore, Michelle Axe, Richard J. Caplan, Jacqueline Ortiz, Mitchell R. Fawcett, Diane C. Bohner, Linda Brittingham, Matthew Hoffman, Nora Katurakes, Mia Papas

\section{ChristianaCare Value Institute, Wilmington, DE}

Background: Addressing patients' social needs is critical for improving health equity. Many health systems are adopting integrative approaches that include screening for and addressing social needs. While acceptance of social needs screening has increased, concerns about time added to clinical workflows persist. Thus, our collaborative of system stakeholders at a Mid-Atlantic health system conducted a study to 1) develop a screener to identify individuals with social needs, and 2) evaluate the sensitivity and specificity of 1- and 2-item screeners in detecting patients with social needs.

Methods: We selected 10 validated screening questions that measured 9 social needs: housing quality, utility needs, housing insecurity, transportation needs, food insecurity, interpersonal violence, health literacy, limited health care access due to cost, and urgent needs. A financial insecurity question was selected as the validation item; this was evaluated alone and in combination with Medicaid status. Data were collected in 1) Complex Care Community, a care coordination service for high-risk patients; 2) obstetric triage (ObTriage); and 3) the Healthy Latinx Families Program (HLFP) and the Latin American Community Center (LACC), two community health worker programs serving the Latinx community. Analyses and validation diagnostics 
were calculated by program.

Results: Complex Care Community $(\mathrm{n}=944)$ and ObTriage $(\mathrm{n}=317)$ patients were largely female, non-Hispanic, English-speaking, White or African American, and Medicaid beneficiaries. HLFP $(n=99)$ and LACC $(n=121)$ participants were largely female, Hispanic, Spanish-speaking, and a race classified as Other; few had Medicaid. Overall, 66\% of Complex Care Community and $40 \%$ of OBTriage patients had more than 1 social need, while more than $90 \%$ of HLFP and LACC participants did. Sensitivity to detect social needs was low for the 1-item screener and improved by adding Medicaid status - $43 \%$ vs $78 \%$ for Complex Care Community, $48 \%$ vs $75 \%$ for OBTriage, $66 \%$ vs $73 \%$ for HLFP, and $48 \%$ vs $57 \%$ for LACC. The addition of Medicaid status decreased specificity to less than $30 \%$ in all except one program.

Conclusion: The 2-item screener was somewhat sensitive but less than $80 \%$. Specificity was low. While our 10-item screener was successful in eliciting social needs, we failed to find compelling evidence that either a 1- or 2-item brief screener would adequately detect patients with social needs.

\section{Addressing Unmet Legal Needs Among Vulnerable Patients: What Is the Impact on Chronic Disease?}

\author{
Alicia L. Salvatore, Mathew D. Walt, Alexandra Mapp, \\ Elsie Evans, Christopher Moore, Erin Booker, Bettina \\ Tweardy Riveros, Cindy Santiago, Dawn Baker
}

\section{ChristianaCare Value Institute, Wilmington, DE}

Background: Medical legal partnership (MLP), addressing patients' unmet legal needs through health systemlegal aid collaboration, is a well-recognized and widely used intervention for underserved and high-need patient populations. Yet, it remains unclear if MLPs improve chronic disease outcomes. In this study, we examined the effectiveness of the Delaware Medical Legal Partnership (DE MLP), a collaboration between ChristianaCare, a MidAtlantic health system, and Delaware Community Legal Aid Society, Inc., on improving systolic (SBP) and diastolic blood pressure (DBP) and hemoglobin A1c (HbA1c) levels. Methods: We used a pragmatic intent-to-treat approach and longitudinal mixed-effects linear regression models to examine changes in chronic disease (SBP, DBP, HbAlc) from the period before and after MLP enrollment.

Results: A total of 215 patients participated in the DE MLP between November 2018 and June 2020. Patients were largely African American (53\%) and White (34\%), non-Hispanic (86.4\%), female (65\%), and had Medicaid or self-pay (53\%). The cohort had a mean of 6.1 comorbidities (standard deviation: 4.1). In unadjusted intent-to-treat analyses among participants with hypertension ( $\mathrm{n}=80)$, MLP participation was associated with significant reductions in SBP (-1.93 mmHg, 95\% CI: $-3.4,-0.39)$ and DBP (-0.93
mmHg, 95\% CI: -1.8, -0.04). Small but not significant decreases in HbA1c were observed among participants with diabetes ( $\mathrm{n}=39 ;-0.19 \mathrm{mmHg}, 95 \%$ CI: $-0.49,0.10)$. Results from adjusted models were not substantially different.

Conclusion: Participation in the DE MLP was associated with significant improvements in blood pressure but not HbA1c. While no similar studies of MLPs could be found, our findings are similar to evaluations of health system interventions to address social needs. These findings are encouraging, as an almost 5\% reduction in relative risk for coronary heart disease events is associated with a $2-\mathrm{mmHg}$ reduction in SBP or a 1-mmHg reduction in DBP. Our study offers evidence that addressing patients' unmet legal needs and their sequalae may benefit cardiovascular health. Multisite controlled studies with larger participant populations and additional health outcomes are needed to elucidate and further strengthen evidence for MLP health impact.

\section{Association of Patient and System-Level Factors in Screening for Social Determinants of Health}

\author{
Samuel Savitz, Mark Nyman, Anne Kaduk, Conor \\ Loftus, Barbara Barry
}

Mayo Clinic, Rochester, MN

Background: Health systems are increasingly recognizing the importance of social determinants of health $(\mathrm{SDoH})$ for care delivery. However, gaps remain in understanding facilitators or barriers to screening for SDoH. In this study, we evaluate a real-world implementation of an electronic health record (EHR)-integrated SDoH screening. The objective was to identify patient and system-level factors that were associated with completion and the modality used. Methods: The study is a retrospective analysis of the implementation of SDoH screening at Mayo Clinic from June 1, 2019, to October 31, 2019. The screening was introduced enterprise-wide on June 1 . We analyzed the first instance when patients were assigned the screening. Outcomes were completion of screening and modality used - MyChart, filled out by patient on patient portal; WelcomeTablet, filled out by patient in the lobby on a tablet; or EpicCare, data obtained directly by provider and entered directly in EHR chart. We conducted logistic regression for completion and multinomial logistic regression for modality. The factors of interest were race/ethnicity, use of an interpreter, whether the visit was for primary or specialty care, and time since implementation.

Results: We identified 500,417 patients who were assigned the SDoH screening, of whom 307,890 (61.5\%) completed the screening. Patients who used an interpreter and racial/ ethnic minorities (compared to White patients) were less likely to complete the screening. Primary care visits were associated with an increase in completion compared to 
specialty care visits. Completion was highest just after implementation and declined in the later periods. Patients who used an interpreter, racial/ethnic minorities, and primary care visits were associated with greater use of WelcomeTablet.

Conclusion: Patient and system-level factors were associated with completion and modality. The lower completion and greater use of WelcomeTablet among patients who use interpreters or racial/ethnic minorities suggests that there is value in having the WelcomeTablets available and that improving the completion rate may be possible by increasing WelcomeTablet availability and addressing workflow issues at the point of care. The higher completion in primary care visits suggests that more training or outreach is needed in specialty care. The decrease in completion over time suggests that sustainment may require continued engagement to support ongoing screening.

\section{Referrals for Social Needs in the Clinical Setting: Pilot Data From 2500 Referrals at 3 Community Hospitals}

\section{Yi Li, Alvia Siddiqi, Rasha Khatib}

\section{Advocate Aurora Research Institute, Downers Grove, IL}

Background: Evidence shows that addressing social needs in the clinical setting leads to improved health outcomes and lower cost. However, initiatives to collect data on social needs are limited, and little is available on the types of community resource patients may need. This study aims to examine referral patterns for social needs in a large Midwestern health system.

Methods: This is a cross-sectional analysis of electronic health records data over a 2-year period (2018-2020) from patients visiting emergency departments or admitted to 1 of 3 community hospitals where a digital platform was piloted to screen and refer patients with needs. The platform includes an inventory of community resources mapped by zip code for referrals. Referrals can be auto-selected based on screening results or manually identified by a careteam member. Referrals were categorized into 22 types of community resources. Patients may be referred to multiple resources. Descriptive statistics and unadjusted risk ratio (RR) with 95\% CI were reported.

Results: Overall, 2496 patients (mean age: $51 \pm 21.6$ years) were referred to at least 1 community resource during the study period. Most patients were African American (66\%) or Caucasian (30\%), and 15\% were Hispanic. About $14 \%$ were uninsured. Nearly half ( $48 \%$ ) were referred to 3 or more resource categories. Top referred categories included financial stability (52\%), food/nutrition (44\%), and transportation $(24 \%)$. The likelihood of receiving 3 or more referral categories was greater for English-speaking vs non-Englishspeaking patients (RR: 1.5 [1.08-2.16]). African American patients were more likely than Caucasian patients (RR: 1.1 [1.04-1.26]). Hispanic/Latino patients were less likely compared to non-Hispanic/Latino patients (RR: 0.9 [0.760.98]). Uninsured/self-pay patients were more likely than patients with commercial insurance (RR: 1.3 [1.16-1.54]).

Conclusion: This evaluation reveals that many patients attending health care have multiple social needs that can be identified at the point of care, and patterns of need-based referral vary by demographic characteristics. More research is needed at the community level to assess if available resources are utilized to address patient needs and at the health system level to examine how addressing these needs impacts patient outcomes.

\section{Social Determinants of Health and Psychosocial Well-Being Since the COVID-19 Pandemic: Findings From the Delaware COVID-19 Community Study}

\author{
Cecelia Harrison, Alexandra Mapp, Mia Papas, Alicia \\ Salvatore
}

ChristianaCare, Newark, DE

Background: The COVID-19 pandemic is impacting the lives of Delawareans in myriad ways that may have longlasting impacts on health and well-being. We conducted the Delaware COVID-19 Community Study (DCCS) to assess the pandemic's impacts on social determinants of health $(\mathrm{SDoH})$ and health and well-being among Delaware adults. In this study, we examined associations between $\mathrm{SDoH}$ and 3 psychosocial well-being outcomes: perceived stress, generalized anxiety, and depression.

Methods: The DCCS was an anonymous, online, crosssectional survey conducted from June 2020 to October 2020. Participants reported sociodemographic characteristics and $6 \mathrm{SDoH}$ (food insecurity, financial insecurity, housing insecurity, utility needs, child care needs, and inability to see a doctor due to cost) experienced before and since the pandemic. We used the Perceived Stress Scale 4 (PSS-4), 2-item Generalized Anxiety Disorder (GAD-2), and Patient Health Questionnaire-2 (PHQ-2) tools to measure perceived stress, generalized anxiety, and depression, respectively. Descriptive and multivariate linear regression analyses were performed; models were age-adjusted.

Results: Participants $(\mathrm{n}=341)$ had a mean age of 52.2 years and were mostly female $(74.7 \%)$, White $(76.1 \%)$, and employed (83.3\%). Most SDoH-related needs reported were higher since the pandemic: $11.1 \%$ were food insecure, $13.8 \%$ were financially insecure, $2.1 \%$ had utility needs, $9.2 \%$ had child care concerns, $16.8 \%$ did not feel physically or emotionally safe at home, and $5.1 \%$ could not see a doctor when needed to due to cost. Food insecurity was positively associated with perceived stress, generalized anxiety, and depression; the latter was significant (PSS-4 $\beta: 1.14, \mathrm{P}=0.13$; GAD-2 $\beta$ : 0.88, $\mathrm{P}=0.08$; PHQ-2 $\beta$ : 0.84, $\mathrm{P}=0.03$ ). Financial 
insecurity had significant positive associations with all three outcomes (PSS-4 $\beta$ : 1.64, $\mathrm{P}=0.02$; GAD-2 $\beta$ : 0.89, $\mathrm{P}=0.04$; PHQ-2 $\beta$ : 0.81, $\mathrm{P}=0.02$ ). Child care concerns also were associated with increases in all outcomes; associations with generalized anxiety and depression were significant (PSS-4 $\beta$ : 0.74, $\mathrm{P}=0.40$; GAD-2 $\beta$ : 1.26, $\mathrm{P}=0.02$; PHQ-2 $\beta$ : $1.04, \mathrm{P}=0.01$ ).

Conclusion: In Delaware, the COVID-19 pandemic has negatively impacted SDoH such as food insecurity, financial security, and child care concerns, which are associated with higher levels of perceived stress, generalized anxiety, and depression. Interventions to address social needs and psychosocial well-being are needed now and should be prioritized alongside vaccination and testing.

\section{Housing Insecurity Among Acute-Care Patients Within a Large Health Care System in Northern California}

Kristen M.J. Azar, Robert Romanelli, Satish Mudiganti, Catherine Nasrallah, Stephanie Brown, Joyce LaMori, Jacqueline Pesa, Anna Kiger, Alice Pressman

\section{Sutter Health Institute for Advancing Health Equity, San Francisco, CA}

Background: As of January 2019, 27\% of people experiencing homelessness in the United States are in California. Research has demonstrated numerous health risks associated with housing insecurity and homelessness $(\mathrm{HI} / \mathrm{H})$. As such, health systems have a unique opportunity to address the unmet needs of these marginalized individuals.

Methods: We studied $\mathrm{HI} / \mathrm{H}$ among acute-care patients at Sutter Health, a large integrated health system in Northern California. From the electronic health record, we identified adults (18+ years old) with and without evidence of $\mathrm{HI} / \mathrm{H}$ between July 1, 2019, and June 30, 2020 and compared their sociodemographic, clinical, and health care utilization characteristics. HI/H data was identified from encounters, flowsheets, and patient address. Comorbidities were identified by ICD-10 diagnoses from problem lists, encounters, or hospital discharge diagnosis; frequent emergency department (ED) use was defined as $>3$ ED visits in any month, or $>5 \mathrm{ED}$ visits within 12 months prior to the first encounter in the study period.

Results: Among 434,286 adults assessed, 20,230 (4.7\%) had evidence of $\mathrm{HI} / \mathrm{H}$. A higher proportion of those with evidence of $\mathrm{HI} / \mathrm{H}$ were male $(66.3 \%$ vs $41.8 \%)$ and Black $(24.3 \%$ vs $11.3 \%)$; the majority were $30-65$ years old (74.6\%). A higher proportion in the $\mathrm{HI} / \mathrm{H}$ group had a diagnosis for serious mental illness (28.4\% vs $17.2 \%)$ but generally had fewer physical comorbidities. Among those with $\mathrm{HI} / \mathrm{H}, 34.7 \%$ were covered by Medicaid, and nearly one-third (32.8\%) had commercial insurance (compared to $17.5 \%$ and $51.3 \%$ without $\mathrm{HI} / \mathrm{H}$ ). Compared to non- $\mathrm{HI} / \mathrm{H}$, a higher proportion of $\mathrm{HI} / \mathrm{H}$ patients were frequent $\mathrm{ED}$ utilizers ( $7 \%$ vs $0.8 \%)$, had a higher frequency of inpatient visits $(11.7 \%$ vs. $6.5 \%)$, and yet were less frequent utilizers of outpatient care (7.9\% vs $29.1 \%)$.

Conclusion: By capturing data on $\mathrm{HI} / \mathrm{H}$, we successfully identified this issue among $4.7 \%$ of the acute-care patients seen over the course of 1 year. The $\mathrm{HI} / \mathrm{H}$ population was disproportionately male and Black and had higher ED and inpatient use but lower outpatient use, suggesting unmet medical needs. These data highlight the need for health system-designed interventions that can connect $\mathrm{HI} / \mathrm{H}$ patients to more consistent preventive care and social services and presents an opportunity for targeted housing interventions and linkage to community-based resources.

\section{Racial/Ethnic Differences in Mortality Among Patients With COVID-19}

Suma Vupputuri, Kevin B. Rubenstein, Celeena R. Jefferson, Sadiya S. Khan

Kaiser Permanente Mid-Atlantic States, Rockville, MD

Background: Black and Hispanic populations have experienced an approximately 2 -fold higher rate of death due to coronavirus disease 2019 (COVID-19) compared with White populations. However, the impact of demographic, socioeconomic, and clinical factors on racial/ethnic differences in death among hospitalized and nonhospitalized patients with COVID-19 is not well known. Therefore, we leveraged longitudinal electronic health record (EHR) data from an integrated health system to describe differences in mortality by race/ethnicity in patients with COVID-19.

Methods: We conducted a retrospective EHR-based cohort study at Kaiser Permanente Mid-Atlantic States among adults ( $\geq 18$ years old) with COVID-19 between January 1 , 2020, and October 31, 2020. Positive COVID-19 patients included those identified via laboratory test or diagnosis. We used Cox proportional hazard models to estimate the hazard ratio (HR) for all-cause mortality in Black and Hispanic patients compared with White patients in unadjusted and stepwise adjusted models to control for demographics, comorbidities, clinical, and behavioral factors.

Results: Among 19,241 patients identified with COVID-19, $40 \%$ were Black, $31 \%$ were Hispanic, $8 \%$ were Asian, and $14 \%$ were White. Mean (standard deviation) age was similar in Black, Hispanic, and White patients (48 [17] years, 45 [14] years, and 48 [19] years). Less than $4 \%$ of Black patients and less than $1 \%$ of White and Hispanic patients lived in neighborhoods with median household income of $<\$ 25,000$. Prevalence of hypertension was highest among Black patients (40\%) compared with Hispanic (22\%) and White patients (28\%). During follow-up, death occurred in $2.3 \%$ of all patients with COVID-19, with $75 \%$ of all deaths occurring in Black and Hispanic patients. Crude HRs for 
mortality were 1.16 (95\% CI: $0.89-1.51)$ and $0.44(95 \%$ CI: 0.31-0.60) for Black and Hispanic patients, compared with White patients. After adjusting for age, sex, and neighborhood income, the HRs were 1.65 (95\% CI: $1.26-$ 2.17) for Black and 1.06 (95\% CI: 0.75-1.50) for Hispanic patients. In fully adjusted models, HRs were 1.59 (95\% CI: 1.05-2.40) for Black and 1.76 (95\% CI: 1.09-2.84) for Hispanic patients.

Conclusion: In a sample of mostly insured patients with access to health care, Black and Hispanic patients experienced a greater burden of COVID-19 mortality. These strong, persistent race/ethnicity-based differences warrant greater focus on social determinants of health as root causes of inequities.

\section{Virtual Recruitment Strategies for Underserved Populations in Rural Pennsylvania}

Tabitha Milliken, Kortney McBryan, Donielle Beiler, Samantha Hoffman, Vanessa Troiani

\section{Geisinger Research, Lewisburg, PA}

Background: Participant recruitment in rural and underserved populations can present unique challenges. These challenges are further exacerbated by the need for low-cost recruiting, which often leads to the utilization of online recruitment methods (eg, email, social media). Social media platforms are predominantly used by younger populations and require access to internet, to which those with lower socioeconomic means and less education are less likely to have access. The current study highlights how a recruitment strategy that utilizes email in combination with a follow-up phone call produced high enrollment rates that included a diversity of participants from rural populations in older age brackets.

Methods: We evaluated the overall enrollment rate of one recruitment arm of a larger study that aims to understand the relationship between genetics and substance use disorders. We evaluated the characteristics of the enrolled population to determine recruitment success of a combined email and follow-up telephonic recruitment strategy, as well as the rate of enrollment of underserved and rural populations. These characteristics included 1) enrollment rate before vs after follow-up phone call, 2) zip code/county of enrollee to determine rural status, and 3) age to verify recruitment in all eligible age brackets.

Results: We found the email and follow-up telephonic arm of the study had a $17.3 \%$ enrollment rate. Of those enrolled, $38 \%$ (2257) of the participants enrolled before the followup phone call (email communication only) and 62\% (3730) of the participants enrolled after a follow-up phone call (email and phone call). Of the enrolled participants, $76 \%$ lived in counties classified as rural and $24 \%$ lived in counties classified as urban in Pennsylvania. Enrolled patients were represented in all eligible age brackets, from ages 18 to 75 years, with the 60-69 age bracket having the most enrollees. Conclusion: Results indicate that combining an online recruitment method with a more traditional method, such as a phone call, yields higher enrollment rates than online methods alone. Adding a humanizing component, such as a live-person phone call, may be a key element needed to establish trust and connection to encourage patients from underserved and rural areas to enroll in studies via virtual recruiting methods.

\section{Implementing a Qualitative Workforce Needs Assessment to Actualize an Equity, Inclusion, and Diversity Workgroup in Kaiser Permanente Southern California's Department of Research \& Evaluation}

\author{
Bhanuja Dub, Alejandra Montano, Carol Flores
}

Kaiser Permanente Southern California, Pasadena, CA

Background: The Kaiser Permanente Southern California (KPSC) Department of Research and Evaluation (R\&E) established a new equity, inclusion, and diversity (EID) workgroup in 2020 in response to the need for broad, sustained activity to address racism and inequities in health and health care in the United States within the workplace. A key early step in the development of the EID workgroup strategic plan was a survey of R\&E employees to assess their current awareness, preferences, and interest in involvement in EID-related activities.

Methods: We developed an online survey seeking suggestions for increasing attention to EID issues in the workplace, enhancing a focus on health equity within research studies and related issues. The survey was initially fielded to a small division within R\&E. After confirming support for the EID initiative through initial responses and after receiving leadership buy-in, the anonymous survey was sent to the entire department $(\mathrm{N}=414$ research and administrative staff). Survey responses were analyzed using qualitative content analysis.

Results: A total of $60 \mathrm{R} \& \mathrm{E}$ staff returned completed surveys (response rate of 14.5\%). Suggestions and comments were rich and diverse and were coded into 6 overall categories of suggested goals and associated activities: 1) Increase workforce diversity; 2) Increase community and stakeholder engagement; 3) Describe and promote EID research attention and appropriate methods; 4) Publicize EID-related funding opportunities; 5) Measure and track health disparities and their determinants through research; and 6) Host EID-related training and events.

Conclusion: The needs assessment survey provided an opportunity for employees to comment on EID issues and offer recommendations anonymously and openly. Survey findings plus guidance from the Institute for Healthcare 
Improvement's framework for health care organizations to improve health equity led to the creation of 3 subcommittees and work streams - capacity building, research, and training and events. Ultimately, the KPSC EID workgroup hopes to foster a diverse and inclusive workplace culture that welcomes open communication and dialogue around EID, to provide department-specific guidance for research teams related to EID issues, and to establish metrics to track EID efforts and their impacts.

\section{Employee-Led Grassroots Initiative in Advancing Equity, Inclusion, and Diversity (EID) in a Health System-Based Research Department: Emergence of the Kaiser Permanente Southern California EID Workgroup}

Carol Flores, Bhanuja Dub, Alejandra Montano, Angelika Alem, Brian Mittman; EID Workgroup Steering Committee

\section{Kaiser Permanente Southern California, Pasadena, CA}

Background: The "dual pandemics" of 2020 - COVID-19 plus heightened awareness of persistent inequities and racism in U.S. society, health care, and health research mandate fundamental and far-reaching changes in health system-based research centers' priorities, studies, and approaches. Recognizing the urgency for action within Kaiser Permanente Southern California's Department of Research and Evaluation (R\&E), 7 female staff from historically marginalized communities wrote an open letter to R\&E leadership proposing a department-wide equity, inclusion, and diversity (EID) initiative to (a) enhance R\&E employees' awareness and attention to EID issues, and (b) foster appropriate responses in our organization's research and workplace culture.

Methods: Following an initial positive response by leadership, the EID organizers engaged key stakeholders (including leadership, scientists, research and administrative staff, and others), fielded a department-wide needs assessment survey, established a governing structure including subcommittees, and launched a program of activities to achieve key goals.

Results: During the initial 6 months of EID workgroup activities, 3 subcommittees (capacity building, research, training and events) were established, with a total of 54 R\&E employees volunteering to join a subcommittee and participate in planning calls. Multiple events were held and a series of EID-related communications disseminated via email. Subcommittee members facilitated 8 small group discussions on race and racism. Two department-wide seminars were held on health equity research and EID in the workplace, with an average attendance of $100 \mathrm{R} \& \mathrm{E}$ staff per session. An EID Sharepoint site was established along with an EID-focused newsletter and monthly EID events calendar showcasing multiple nationwide relevant activities. R\&E leadership incorporated EID trainings into departmental goals, focusing on fostering a positive work environment.

Conclusion: Health system-based research centers possess unique opportunities to design, evaluate, and spread innovative solutions to inequity and racism in health, health care, and society. The R\&E EID workgroup offers a valuable model for other research centers interested in enhancing researchers' awareness of, and attention to, health inequities and racism. Supportive leadership and a committed group of internal stakeholders are key to success in efforts to encourage health system researchers to contribute to meaningful efforts to counter decades of damaging policies, practices, and beliefs.

\section{Social Needs Data Collection for Complex Care Members}

Musu Sesay, Doraina Walker-Williams, Marni Segall, Teaniese Davis, Drew Bradlyn

\section{Kaiser Permanente Georgia, Atlanta, GA}

Background: Addressing nonmedical needs is essential for quality care delivery in assessing a patient's overall health and well-being. Nonmedical needs may be most salient among older adults, some of whom are retired, may have chronic health conditions or disabilities, or may have limited financial resources. Our objective was to better understand short-term nonmedical needs within our complex care population.

Methods: In 2019, we invited 6021 Kaiser Permanente (KP) Georgia members who were $\geq 60$ years of age with $\geq 3$ chronic comorbid conditions to complete an adapted version of the Your Current Life Situation (YCLS) survey via email and mail. The YCLS survey, administered at baseline and at 6 months postbaseline, included questions about needs related to food, housing, transportation, utilities, intentions to seek assistance for needs if offered at no cost, and attitudes toward sharing needs with health care providers.

Results: A total of $158 \mathrm{KP}$ members participated in the study, and $94.3 \%(\mathrm{n}=149)$ participants completed both baseline and follow-up surveys. Participants were predominantly nonHispanic (93.7\%), White (68.4\%), and female (59.5\%), with at least a high school diploma (96.2\%). Mean age was 74.7 years, and Charlson score mean was 5.42. Approximately $27 \%$ of participants indicated a nonmedical need at baseline $(\mathrm{n}=42)$ or follow-up $(\mathrm{n}=41)$. Compared to baseline, at 6-month follow-up there was an increase in participants who indicated trouble with transportation and housing and a decrease in reporting trouble with food and utilities. For participants who completed both baseline and follow-up, $16.8 \%(\mathrm{n}=25)$ indicated at least one need at both timepoints, of which $40 \%(n=10)$ reported an increase and $40 \%(n=10)$ reported no change in number of needs. The majority of 
participants reporting a need at baseline and follow-up indicated they would seek assistance to address their need(s) if offered at no cost and would want their provider to know about their needs.

Conclusion: While the survey indicated a decrease in some needs, there were increases in other areas for nonmedical assistance among complex care patients. Participants want their health care providers to know about their need(s) and are interested in seeking assistance to address need(s) if offered at no cost. Patients are amenable to provider engagement in screening for nonmedical needs.

\section{Participant Perspectives on Social Needs Data Collection}

Musu Sesay, Doraina Walker-Williams, Marni Segall, Teaniese Davis, Drew Bradlyn

\section{Kaiser Permanente Georgia, Atlanta, GA}

Background: Addressing social (nonmedical) needs is an important consideration for patients' overall health and wellbeing. Screening older adults by asking about nonmedical needs will help with gaps in quality care delivery and reduces hesitation in discussing with health care providers. Our objective was to understand members' experiences, barriers, and facilitators associated with social needs assessment and resource referrals.

Methods: This is part of an explanatory mixed-methods study following baseline and 6-month assessment of social needs using an adapted version of Kaiser Permanente (KP)'s Your Current Life Situation (YCLS) survey. We conducted 2 focus groups and 14 interviews with select enrolled study participants (KP Georgia members, $\geq 60$ years of age, with $\geq 3$ chronic comorbid conditions) to provide feedback about the assessment of social needs, the YCLS questions, and experiences with a social needs resource guide. Participants were categorized based on their YCLS responses for those who reported: 1) no needs $(\mathrm{n}=5) ; 2)$ a need but sought no assistance $(\mathrm{n}=5)$; and 3$)$ a need and sought assistance $(n=4)$. Discussions focused on participants' needs and challenges, opinions on the YCLS survey, and usefulness of the resource guide.

Results: In all, 21 members (8 males and 13 females) participated. Participants expressed comfort in answering questions about their needs and found the YCLS survey useful. They did not consider the YCLS questions to be "prying," with the exception of 1 participant who expressed initial hesitancy, fearing negative consequences to health benefits. Several participants indicated that, "If you do not know what we need, how would you help us?" Participants noted no preference for telephone vs online completion of the survey, since their most important consideration was trust of the person or organization asking the questions and they considered KP a trusted source. Participants expressed openness in discussing their needs. However, they reported reservations about reaching out for help and possibly burdening their health care providers and a preference for working with family members to address their needs.

Conclusion: Participants welcomed the idea of their health care providers screening for nonmedical needs, providing them with information to obtain assistance for those needs if needed, and expressed receptiveness to such assistance if provided at no cost.

\section{Perceived Value of Resource Referral Among Complex Care Members}

\author{
Doraina Walker-Williams, Marni Segall, Musu Sesay, \\ Tina Davis, Derw Bradlyn
}

\section{Kaiser Permanente Georgia, Atlanta, GA}

Background: Providing resources to effectively address nonmedical needs and improve the health and well-being of communities is an important undertaking by governmental and nonprofit agencies. This assistance is vital to older adults who encounter social and financial limitations to receiving necessary help. The purpose of this study was to provide and understand the utility of providing a local resource guide for older adults seeking nonmedical assistance.

Methods: Our team developed a resource guide using online database resources compiled by the United Way of Greater Atlanta and the American Association of Family Physicians. The guide was a component of a mixed-methods social needs study designed to examine changes in nonmedical needs. Kaiser Permanente Georgia's complex care members who were 65 years of age or older and had 3 or more chronic comorbid conditions were eligible to participate. Resource guides were sent to 238 eligible members (irrespective of study interest) who requested one from an initial outreach soliciting study participation. Of the eligible members, 149 enrolled study participants completed the follow-up survey evaluating the usefulness of the resource guide. Guides included agencies serving 15 metro-Atlanta counties, were tailored by zip code, and outlined agency contact information, description, service eligibility requirements, hours, and associated costs. Resources included assistance with food (pantries, food delivery, soup kitchens), housing, transportation, and utilities.

Results: Overall, $51 \%(\mathrm{n}=76)$ of participants acknowledged receiving the resource guide. Of those, $72.3 \%(n=55)$ reported the guide was helpful overall; $56.6 \%(n=43)$ found the guide helpful or very helpful for assistance; and 13.2\% $(\mathrm{n}=10)$ used the guide to secure assistance for their needs. More than one-third $(34 \%, \mathrm{n}=26)$ shared the local resource guide with someone (spouses, children, friends, others), and $10.5 \%(\mathrm{n}=8)$ reported that person used the guide to secure assistance for their needs.

Conclusion: Members found the resource guide helpful 
in seeking assistance for nonmedical needs. This study highlights the utility of a more passive approach to providing resources for patients with social needs. While approximately half the sample acknowledged receiving the resource guide, health systems still need to consider multiple approaches to linking patients to resources who have social needs.

\section{IMPLEMENTATION SCIENCE}

\section{Integration of Guideline Revisions Into Provider Mindlines and Treatment Decisions}

\author{
Arwen Bunce, James Davis, Rachel Gold
}

\section{OCHIN, Inc., Aurora, CO}

Background: A study examining the influence of implementation support on adoption of guidelineconcordant cardioprotective prescribing in community health centers (CHCs) began shortly after a major change in guideline recommendations regarding the use of statins for primary prevention of cardiovascular disease. This change recommended replacing specific cholesterol treatment goals with fixed statin doses based on individual risk. This analysis explored how the new guidelines were integrated into providers' mindlines, ie, tacit and experiential knowledge influencing their treatment decisions and prescribing practices.

Methods: Over 3 years (2015-2018) researchers interviewed 43 providers at 20 clinics. Interviewees were asked if their prescribing approach had shifted due to the guideline changes, how they introduced statins to their patients, and what might cause them to not prescribe statins; these topics also arose organically. Interviews were transcribed and coded in QSR NVivo; analysis followed the constant comparative method.

Results: The extent to which the revised guidelines shifted providers' mindlines (and therefore prescribing practices) fell into 3 general categories: 1) Those who incorporated the new guidelines into their decision-making and explicitly discussed individual risk when talking with patients; 2) Those who believed in the new approach in theory but found it troublesome to implement in practice; and 3) Those who continued to prescribe based solely on cholesterol-level targets. Providers identified multiple factors that influenced their concordance with the revised statin guidelines (ie, mindlines), including: a) familiarity with the new guidelines; b) views on the applicability of population-based guidelines to individual patients, particularly $\mathrm{CHC}$ patients; c) beliefs regarding the interplay of professional autonomy and treatment guidelines in patient care; d) intuition/gut feeling; e) comfort with prescribing a lifelong medication to younger or seemingly healthy patients; f) practice inertia; g) trust in the underlying evidence; and h) deference to specialists.
Conclusion: Evidence-based clinical guidelines are notoriously difficult to embed into clinical practice. Enacting new guidelines requires a shift in individual clinicians' mindlines, which are shaped by personal experience, training, values, and interactions with others as well as evidence-based research. Guideline-implementation efforts should address the multiple knowledge sources clinicians draw on when making treatment decisions.

\section{Supporting Community Health Center Patients in Completing Follow-Up Colonoscopy: A Qualitative Summary of Patient Navigation Activities}

Jennifer S. Rivelli, Jennifer L. Schneider, Jamie Thompson, Amanda Petrik, Neha Yadav, Leslie Mosso, Edward Perez, Gloria Coronado

\section{Kaiser Permanente Northwest, Portland, OR}

Background: Fecal immunochemical tests (FITs) are an acceptable and cost-effective way to identify patients at risk for colorectal cancer. However, patients who receive an abnormal FIT result must obtain a follow-up colonoscopy. Rates of follow-up colonoscopy are low in most health care settings, particularly in Federally Qualified Health Centers (FQHCs). Patient navigation can provide much needed support for patients to complete the follow-up colonoscopy. Methods: PRECISE is a patient-randomized trial of patient navigation vs usual care for follow-up colonoscopy implemented within a FQHC in Washington State (anticipated 37\% Latinx). A bilingual (native Spanish speaker) patient navigator was trained based on the New Hampshire Colorectal Cancer Screening Program. As part of the recruitment and data tracking tool used by the patient navigator, open text fields are available for documenting interactions with navigated patients. We present a qualitative content analysis of the open text fields of navigated patients following the first 8 months of the study. These findings identify the range of supportive interactions engaged in by the patient navigator to assist patients in overcoming barriers to follow-up colonoscopy completion.

Results: A total of 52 participants had open text field documentation, representing both decliners $(n=8)$ and those navigated to colonoscopy completion $(n=44)$. Analysis of the 44 navigated patients revealed common areas where the navigator provided support, including contacting the gastroenterologist office to problem-solve issues like lack of referral, scheduling delays, or to obtain appointment information (40 instances); assisting the patient with obtaining, understanding, and completing the bowel preparation (25 instances); and verifying, coordinating, and offering transportation/escort support before and after the colonoscopy procedure (22 instances). For the 8 decliners, most had multiple reasons for declining, including general resistance/fear, other health priorities, desiring a second FIT 
test, insurance challenges, and prior negative experience with the bowel preparation formula. One participant declined navigation due to having prior colonoscopy experience.

Conclusion: The PRECISE trial highlights the importance of targeted patient navigation to improve colorectal cancer screening completion. Our open text field findings demonstrate the necessary role of patient navigation for overcoming the multitude of educational and structural challenges facing $\mathrm{FQHC}$ patients when attempting to complete a follow-up colonoscopy.

\section{Comparing Two Approaches to Implementing Suicide Risk Identification Models}

\section{Scott Stumbo, Jennifer L. Schneider, Rebecca Rossom, Julie Richards, Bobbi Jo Yarborough}

\section{Kaiser Permanente Northwest, Portland, OR}

Background: Machine learning models using electronic health record data to identify individuals at risk for attempting or completing suicide are being developed and readied for implementation. A relatively new innovation in suicide prevention efforts, the implementation of these models warrants study; guidelines for acceptable and safe implementation do not yet exist.

Methods: Guided by the Consolidated Framework for Implementation Research, we interviewed 40 clinicians and health care administrators at 3 integrated health systems. Two sites are currently engaged in implementing suicide risk identification models: one site implemented their approach as part of a mental health visit, the other site integrated it into an existing telephone-based case management program. Clinicians and administrators at the third site, planning for future suicide risk identification model implementation, also were interviewed about their concerns and hopes for this technology.

Results: Content analysis of interviews revealed clinicians across sites broadly support the use of suicide risk identification models as another important tool in the suicide prevention toolkit. Clinician recommendations included early engagement of clinicians in decision-making and planning, establishing a clear clinic workflow, and providing appropriate, thorough, and ongoing training. Studying two different implementation approaches elicited different strengths and challenges. For example, the strength of an established patient-provider relationship in a visit-based approach could be compared to the opportunity to reach patients with an outreach approach and the accompanying barrier of not having a history with the patients. Other barriers included competing demands and time and lack of comfort/training in suicide risk assessment. Clinicians across sites called for consolidating all suicide-related risk information into a standardized and easy-to-access module in the electronic health record to enhance efficiency for risk identification follow-up. Finally, clinicians expressed concern that some patients would still be missed, and they were not sure what to do with patients with chronic suicide ideation who are continuously identified.

Conclusion: Lessons from two sites provided several areas of opportunity for improving implementation of machine learning models that identify suicide risk.

\section{Using Qualitative Comparative Analysis in Implementation Science: Findings and Challenges}

\author{
Inga Gruß, Arwen Bunce, James Davis, Joanna \\ Bulkley, Rachel Gold
}

Kaiser Permanente Northwest, Portland, OR

Background: Identifying determinants of practice change in health care is central to implementation science. Qualitative comparative analysis (QCA) offers a rigorous approach to identify determinants, or "conditions," of practice change by systematically comparing cases (units of analysis) as configurations of conditions of desired outcomes. Using data from a trial comparing implementation support strategies' impact, we performed crisp-set QCA to identify sets of conditions associated with significant improvements in the targeted outcome (guideline-concordant cardioprotective prescribing) in the study clinics. We also reflect on challenges involved when using this methodology.

Methods: Qualitative (staff interviews, observations) and quantitative (electronic health record, surveys) data were collected over 3 years from 29 community health centers across 12 service areas in the United States. Performing QCA involved 4 steps: building a configurational model by selecting 4 conditions of interest; constructing the empirical sample to include all relevant cases (each service area); calibrating the data by translating data into binary categories (present and absent); and analyzing the "truth table" created during calibration to calculate consistency (extent to which a given configuration of conditions is associated with outcome) and coverage scores (empirical relevance of sets of conditions).

Results: The QCA identified 2 conditions as necessary for achieving significant change in the targeted outcome the presence of a study champion (consistency score: 1.00; coverage score: 0.72 ) and the absence of a perceived culture of quality improvement (consistency score: 1.00; coverage score:1.00). No other conditions were necessary or sufficient. Further exploring the finding that the absence of a perceived culture of quality improvement was a necessary condition was challenging because 1) it was not feasible to meaningfully operationalize all conditions that might explain this finding, and 2) adjusting the configurational model post hoc would have required including additional conditions that were not assessed systematically during data collection.

Conclusion: QCA methods can support empirical explanation of implementation research findings, with limitations. In implementation science, important conditions 
that contribute to implementation success or failure often are only understood upon completion of a trial. This poses a challenge for systematically collecting data for designing the most appropriate configurational model.

\section{Using Implementation Science to Identify Clinician-Reported Barriers to Discussing Safe Fish Consumption During Routine Prenatal Care}

Meghan JaKa, Jennifer Dinh, Thomas Kottke, Jacob Haapala, Patricia McCann, Jeanette Y. Ziegenfuss

\section{HealthPartners, Bloomington, $M N$}

Background: Fish consumption during pregnancy is beneficial for mothers and developing fetuses; however, care should be taken to choose low-mercury fish. The complexity and importance of this message make it ideal for clinician discussions during routine prenatal care. This project measures clinician-reported behaviors and barriers related to fish consumption conversations, guided by implementation science theory (the Capability, Opportunity, and Motivation Behaviour, or COM-B, model).

Methods: A representative sample of 400 obstetrics/ gynecology and family medicine clinicians across Minnesota were surveyed using a sequential, mixed-mode design with a small token incentive. Questions asked how often clinicians discussed fish consumption and the prevalence of various barriers to these discussions. Reported behaviors and barriers were categorized into the COM-B model. Descriptive and chi-squared statistics were used to report prevalence and differences across clinician characteristics. Responses to open-ended items were coded into COM-B categories using content analysis.

Results: A total of 219 clinicians completed the survey (55\% response rate), with no differences by specialty or geography. Most clinicians reported the "behavior" of discussing nutrition generally, but the most common topic was weight management (89\%), with only $35 \%$ discussing the benefits of fish and $27 \%$ discussing risks. Aligning with other COM-B constructs, commonly reported "capability" barriers included knowledge $(67 \%$ did not strongly agree it was important for pregnant women to eat fish); "opportunity" barriers related to context and resources (82\% said not enough time, 63\% said topic not built into encounters, $41 \%$ said topic was not a leadership priority), and "motivation" barriers included lack of perceived professional role $(91 \%$ said dietitians were an ideal source for patient diet information). Qualitative responses reiterated these findings, adding additional context, "It's not prioritized by my employer, perhaps because it's not related to insurance company compensation." Differences by clinician characteristics also will be presented.

Conclusion: Opportunities exist to increase clinician recommendation of healthy and safe fish consumption during pregnancy, but a number of barriers across all COM-B model domains constrain the frequency of these conversations. This presentation will discuss the implications of this work and how results may be used to develop theory-informed intervention strategies to facilitate effective, high-quality informationsharing about fish consumption around the point of care.

\section{Impact of a Multilevel Acute Kidney Injury Rehabilitation Program on Postdischarge Mortality and Rehospitalization}

Gurmukteshwar Singh, Yirui Hu, Steven Jacobs, Jason Brown, Jason George, Maria Bermudez, Kevin Ho, Jamie Green, H. Lester Kirchner, Alex Chang

\section{Geisinger Research, Danville, PA}

Background: Hospitalization-associated acute kidney injury (AKI) is common and associated with markedly increased mortality and morbidity. The objective of this study was to examine the impact of a multilevel AKI rehabilitation program on postdischarge outcomes.

Methods: An AKI rehabilitation program was piloted at an academic center in central Pennsylvania from September 19, 2019, to February 29, 2020. Hospitalized patients with stage 2-3 AKI were identified in real-time using an electronic health record-embedded algorithm and offered enrollment into an AKI rehabilitation program. Patients who had hospice/ comfort care status, unclear goals of care at discharge, or significant cognitive impairment were excluded. The intervention included patient education, case manager services, and expedited nephrology appointments starting within 2-4 weeks of discharge. We examined the association between AKI rehabilitation program participation and risks of rehospitalization or mortality in logistic regression analyses adjusting for comorbidities, discharge disposition, sociodemographics, and kidney parameters. Sensitivity analysis was performed using propensity score matching.

Results: In total, 52 high-risk AKI patients were enrolled and compared to 400 nonparticipants. Crude postdischarge rates of rehospitalization or death were significantly lower for participants vs nonparticipants at 30 days $(15.4 \%$ vs $34.2 \% ; \mathrm{P}=0.01)$ and at 90 days $(32.7 \%$ vs $50.5 \% ; \mathrm{P}=0.023)$. After multivariable adjustment, AKI rehabilitation program participation was associated with lower risk of rehospitalization or mortality at 30 days (odds ratio: $0.42,95 \%$ CI: $0.17-0.95$ ), with similar findings at 90 days (odds ratio: $0.54,95 \% \mathrm{CI}$ : $0.25-1.14)$. In propensity score-matched sensitivity analyses of 46 participants and nonparticipants, risks of rehospitalization or mortality were not significantly different at 30 days (15\% vs $26 \% ; \mathrm{P}=0.3)$ or at 90 days $(37 \%$ vs $35 \% ; \mathrm{P}=1.0)$.

Conclusion: Participation in an AKI rehabilitation program was associated with decreased 30-day risk of rehospitalization or death. Randomized trials are needed to evaluate the efficacy of this strategy. 


\section{Assessing Challenges to Implementing Tobacco Cessation Guidelines in Dental Offices}

Mark W. Jurkovich, Steve E. Asche, Chris J. Enstad, Anjali R. Truitt, Emily C. Durand, Kimberly S. Johnson, Lauryn M. Davin, Heiko Spallek, Jeanette Y. Ziegenfuss, D. Brad Rindal

\section{HealthPartners, Bloomington, MN}

Background: Tobacco use has a negative impact on oral health. Practice guidelines encourage dental providers to provide tobacco using patients with cessation counseling. In order to improve adherence to these guidelines, it is important to understand the factors that make implementation difficult. As part of a clinical trial testing a clinical decision support tool to support guideline compliance in dental practice, we examined baseline factors that could serve as barriers to guideline implementation.

Methods: We enrolled 29 dental providers into a tobacco cessation trial. At baseline we administered a questionnaire based on key theoretical domains relevant to implementation behaviors of health care providers. The online baseline survey was collected from 10 dentists and 19 hygienists at 10 private practice dental offices from July 2019 to August 2020. Descriptive statistics compare endorsement of individual survey items (response scale: $1=$ strongly disagree, $5=$ strongly agree) and patterns of differences in dentist and hygienist responses are described.

Results: Clinicians believe there is value in (mean: 4.1), and that their role includes provision of $($ mean $=4.3)$ tobacco cessation services. Hygienists were directionally more likely than dentists to agree that their role involved assisting patients to stop tobacco use (means: 4.6 vs 4.0). Clinicians had a moderate level of confidence in how to assess patients in efforts to stop tobacco use (mean: 3.5 ) and that they knew the right questions to ask patients (mean: 3.2). Clinicians were less confident in prescribing medications for those ready to quit (mean: 1.9) and had moderate levels of understanding the risks and benefits of nicotine replacement therapy (mean: 2.8). Clinicians lack a system to cue/prompt counseling against tobacco use (mean: 2.1) and report that clinics had done little to remove barriers to the provision of tobacco use counseling (mean: 2.3). Directional differences in dentist vs hygienist item means indicated that receipt of sufficient reimbursement for promoting tobacco abstinence was more of a concern for dentists than hygienists (means: 2.4 vs 3.6).

Conclusion: Dental providers believe they have a valuable role in tobacco cessation. Opportunities to enhance tobacco cessation include providing tools to prompt counseling and assist prescribing medications. Dentists more than hygienists identify reimbursement as insufficient. More could be done to remove barriers.
Developing a Prototype to Promote Data Collection of Sexual Orientation and Gender Identity Through Education and Electronic Health Record Feedback Reports

Christopher Kabir, Cynthia Lira-Crame, Belinda Benitez, Brooke Pichen, Oscar Zambrano

Advocate Aurora Health, Chicago, IL

Background: Collecting sexual orientation and gender identity (SOGI) information is essential to improving patient-centered care, according to the Centers for Disease Control and Prevention. However, obtaining SOGI data in the electronic health record (EHR) is often difficult within large health systems, and information on sexual and gender minority patients remains largely uncaptured and unused by health systems. One reason for this may be a gap in cultural competence in discussing SOGI information with patients. A solution identified by our Lesbian, Gay, Bisexual, Transgender, and Queer (LGBTQ) Task Force was to integrate education with SOGI data extracted from the EHR. In this presentation, we describe the implementation and results of a quality improvement project designed to address barriers in documentation and improve data quality.

Methods: An interdisciplinary committee was established within the LGBTQ Task Force with the mission to reinforce the importance of SOGI information and promote data collection and education throughout the institution. To accomplish this, education material is coupled with visualizations of the institution's SOGI data and shared with nursing leadership at one metropolitan Level 1 trauma center. We also received feedback through anonymous surveys to examine barriers in collecting SOGI information. Results: Our transition to a unified EHR system provided an opportunity to develop and demonstrate a prototype for a standard and user-friendly reporting function that can be accessible throughout the health system. Once this work is complete, we hope to better understand and overcome barriers, as well as improve SOGI data completeness. The committee garnered support from institutional leaders and multiple departments to transition the project into the future state.

Conclusion: To identify and address health disparities, we need to provide ongoing feedback and reinforce the priority of collecting SOGI data at multiple points of care within the clinical workflow. Improved access and flexibility in data visualization to unit-level granularity of EHR data is the first step to identifying gaps and target areas for education. Furthermore, high completion rates of SOGI information throughout the health system is needed and can lead to improved compassionate care for sexual and gender minority patients. 
Beyond Implementation: Developing and Utilizing a 5-Factor Scale to Measure Optimization of Lynch Syndrome Tumor Screening Programs in Health Systems

Zachary Salvati, Deborah Cragun, Victoria Schlieder, Jamie Atondo, Ilene Ladd, Jasmine Burton-Akright, Jessica Ezzell Hunter, Mara Epstein, Christine Y. Lu, Pamala A. Pawloski, Ravi Sharaf, Su-Ying Liang, Andrea Burnett-Hartman, Alanna Kulchak Rahm

\section{Geisinger Research, State College, PA}

Background: Implementation of universal tumor screening (UTS) programs for Lynch syndrome remains variable when comparing health systems nationwide. The IMPULSS study aims to better understand this variability and identify elements that facilitate or impede program optimization.

Methods: The IMPULSS study utilized purposive sampling of 9 health systems ( 7 in HCSRN, 1 academic, 1 national system) with variability in UTS implementation. Semistructured interviews with 44 relevant stakeholders were conducted. Data collection and thematic analyses were guided by the Consolidated Framework for Implementation Research (CFIR) with the intent to identify domains and constructs differing across sites that have successfully implemented and optimized UTS using cross-case comparisons of the data matrices generated.

Results: Some sites had multiple unique UTS operations under the same organizational umbrella, each considered separate analytic units (AUs). To date, we have identified 14 distinct AUs within 6 health systems. Of these, 10 AUs had implemented a UTS program, represented by 31 interviewed stakeholders involved in direct-patient care and/or administrative leadership; 13 stakeholders were interviewed from the 4 AUs without a program. Thematic coding guided by CFIR found differences primarily in constructs within the "Innovation Characteristics" CFIR domain. A 5-point UTS optimization scale was created to indicate presence or absence of each of the following factors important to program optimization: 1) systematic tracking; 2) procedural uniformity; 3) process for "bridging the gap" between tumor screening and germline testing; 4) automatic ordering of appropriate reflex testing; and 5) using data and quality assurance methods to determine and improve program efficacy. By scoring each factor as 1-present or 0 -absent, progress toward UTS program optimization was calculated and areas that need improvement were identified. AUs that scored a 4 or 5 on optimization demonstrated high quality and consistency in "Networks \& Communication." Conclusion: Identification and stratification of CFIR domains and constructs allowed the study team to separate out the processes and factors pertinent to initial implementation and program optimization. We subsequently identified 5 key factors important to UTS program optimization. This will allow us to tailor toolkits to assist with both implementation and optimization of UTS programs.

\section{MATERNAL, CHILD, AND FAMILY HEALTH}

\section{Understanding the Time Costs of Caring for Children With a Chronic Health Condition: A Report From the r-Kids Study}

Frances Lynch, Alexandra Varga, Joanna Bulkley, Lisa Croen, Eric Fombonne, Brigit Hatch, Yihe Daida, April Lee, Maria Massolo, John Dickerson

\section{Kaiser Permanente Northwest, Portland, OR}

Background: All families experience time demands related to caring for their child's health. However, families of children with autism spectrum disorder (ASD) or other chronic health conditions may face a higher burden.

Methods: We used electronic health records from 3 Kaiser Permanente regions (Northwest, Hawaii, and Northern California) and several community health clinics to identify children between 3 and 16.5 years of age with ASD, asthma, or neither (ie, control group). We oversampled racial and ethnic minorities and matched asthma and control groups to the age and gender distribution of the ASD group. Parents or guardians completed online surveys 3 times over 12 months in English or Spanish. Surveys included the Family Economic Impact Inventory (measuring financial, time, and employment costs of caring for a child's health) and instruments measuring the child's quality of life (per PedsQL) and symptom severity for children with ASD (per SRS) or asthma (per PROMIS Asthma), parenting stress, and parent physical and mental health.

Results: In all, 1461 families enrolled in the study ( $\mathrm{n}=564$ ASD, $n=468$ asthma, $n=429$ control). The children had a mean age of 9.1 years and were predominantly male (79\%) and ethnically diverse (21\% Hispanic, $43 \%$ non-Hispanic White, and 35\% non-Hispanic Asian, Black, Native Hawaiian, or other race/ethnicity). Families of children with ASD spent more time coordinating with and attending meetings with schools (mean [standard deviation]: 28 [55] hours in ASD group vs 23 [54] hours in asthma group or 10 [32] hours in control group; $\mathrm{P}<0.0001$ ) and child care providers (mean [standard deviation]: 19 [66] hours in ASD vs 7 [31] hours in asthma or 3 [24] hours in control; $\mathrm{P}<0.0001)$. Families of children with ASD also spent much more time in caregiving tasks at home (eg, managing insurance, coordinating with schools) related to their child's health compared to either group (mean [standard deviation]: 93 (151) hours in ASD vs 13 [59)] hours in asthmas or 10 [46] in control; $\mathrm{P}<0.0001$ ).

Conclusion: Families of children with ASD experience significant time costs related to caring for their child's health. These time demands can have a profound effect on parent stress and ability to function in work and other activities. More attention to these costs could improve policies to support families of children with ASD. 
Association of Adverse Perinatal Outcomes With Maternal Obesity and Gestational Diabetes

\author{
Vani Movva, Kajal Angras, Amanda Young, A. Dhanya \\ Mackeen
}

\section{Geisinger Research, Danville, PA}

Background: The risk of development of gestational diabetes mellitus (GDM) increases with maternal body mass index (BMI). Several adverse perinatal outcomes are associated with both maternal obesity and GDM. The aim of this study was to investigate the concomitant effects of GDM and maternal obesity on adverse perinatal outcomes at term.

Methods: This is an institutional review board-approved retrospective cohort study of women who delivered a singleton at a tertiary care center between 2010 and 2019. We excluded patients with pregestational hypertension or diabetes. Women were categorized by their pregravid BMI (underweight and normal: $<25.0$, overweight: 25.0-29.9, obese: $>30.0 \mathrm{~kg} / \mathrm{m}^{2}$ ) and presence/absence of GDM. To determine if there is an association between obesity and GDM with perinatal outcomes, general linear models were applied for the continuous outcomes and logistic regression for the binary outcomes. Test of trend P-values were reported for all outcomes.

Results: Of the 30,632 included women, $25.1 \%$ were overweight and $31.7 \%$ were obese prior to pregnancy. The risk of GDM was $3.6 \%, 6.1 \%$, and $11.2 \%$ among the underweight/normal, overweight, and obese BMI categories, respectively. Maternal outcomes, including incidence of hypertensive disorders of pregnancy and cesarean delivery, and neonatal outcomes, including APGAR score of $<7$ at 5 min, intensive care unit (ICU) admission, and neonatal jaundice and hypoglycemia, increased with increasing BMI and presence of GDM, with the highest risk in obese patients with GDM $(\mathrm{P}<0.01)$. The gestational age at delivery decreased as BMI increased $(\mathrm{P}<0.01)$; however, it was not clinically significant. Incidence of preterm delivery, shoulder dystocia, length of neonatal ICU admission, and respiratory distress syndrome did not show a significant trend. The risk of stillbirth could not be assessed with only 1 fetal demise in the cohort.

Conclusion: Obesity increases the risk of GDM and worsens outcomes as compared to GDM alone. The test of trend indicated that an increase in BMI class in addition to GDM increased the risk of both maternal and neonatal morbidity. Preventive strategies for risk reduction, such as healthy weight loss prior to pregnancy, should be strongly encouraged by health care providers.
Romantic Relationship and Parenting Distress Associations With Emotional Eating and Alcohol Consumption During COVID-19

\author{
Megan Ferriby Ferber, Stephanie Malzahn, Dixie Meyer \\ Saint Louis University / SSM Health, St. Louis, MO
}

Background: The COVID-19 pandemic shifted work and home life for many American couples and families. Changes such as added demands and responsibilities may elicit coping strategies and, for many, can overwhelm coping skills. Prior research suggests maladaptive coping mechanisms are utilized when negative interactions are triggered between partners and when emotional distress becomes too great to tolerate. This study aimed to examine how individuals are coping with the stressors associated with the COVID-19 pandemic within the relational context. We specifically assessed association of the maladaptive coping mechanisms alcohol use and emotional eating with romantic and family relationship interactions and stress.

Methods: This longitudinal study, part of a larger ongoing study, utilized ecological momentary assessment. A total of 88 participants completed surveys examining relationship satisfaction, daily distress, negative partner interactions, parenting stress, emotional eating, and alcohol use on both weekday and weekend days. Data were analyzed using 4 multiple linear regressions for each maladaptive coping mechanism and for each day assessed.

Results: Results revealed greater use of emotional eating was associated with higher perceived relationship satisfaction $(t=2.19, \mathrm{P}=0.03)$ and parenting distress $(t=2.68$, $\mathrm{P}=0.01)$ during weekdays. Weekend data showed parenting distress $(t=2.52, \mathrm{P}=0.02)$ and daily distress $(t=2.13, \mathrm{P}=0.04)$ were significantly related to greater use of emotional eating. Greater use of alcohol use was associated with parenting distress $(t=2.44, \mathrm{P}=0.02)$ on weekends only.

Conclusion: These results highlight the taxing impact of the COVID-19 pandemic on couples' and families' ability to cope with parenting stress. The limited resources currently available to couples and families require alternate coping strategies be implemented to reduce stress activation. Greater emotional eating (both days) and alcohol use were found to be related to parenting distress, indicating parents may be a particularly vulnerable group to the stress of COVID-19. With the frequency of emotional eating and alcohol use to cope with stress comes increased health risks and the necessity for implementing health care interventions and behavioral approaches that result in more adaptive stress-coping strategies. 


\section{Improving Lead Screening in the Era of COVID-19: Development of a Lead Screening Program in an Integrated Health System Serving Predominantly Rural and Suburban Pediatric Patients}

Karina Phang, Jennifer Vodzak, Stacey Cummings, Heather Mosley, Keith Boell

\section{Geisinger Research, Pittston, PA}

Background: Lead exposure remains a common, preventable environmental health threat to children. Lead screening with blood lead level (BLL) testing is recommended as part of well-child examinations. COVID-19 has adversely affected identification of children with elevated BLL, lead exposure elimination, and linkage to community services and interventions in the United States. Geisinger is a large integrated health system with an outpatient pediatric network serving predominantly suburban and rural children in more than 40 Pennsylvania counties, many with high risk for lead exposure.

Methods: We assembled a multidisciplinary team representing general pediatrics, quality and safety, informatics, laboratory medicine, pharmacy, patient experience, and population health. Our overall aim was to develop a comprehensive pediatric lead screening program that 1 ) systematically performs lead screening, 2) delivers a standardized guideline-adherent response to results, and 3) communicates lead screening and interventions to families using health literacy-informed approaches. We utilized mixed-method quality improvement and implementation science methodologies. We validated lead screening completion metrics utilizing the Cerner HealtheIntent Registry tool. Primary measures included completed BLL at 1-year well visit, completed BLL in 2-year-olds with public insurance, and documentation of lead risk assessment. We completed qualitative assessments of existing patient education and communication strategies.

Results: A key driver diagram outlined stages of program development and intervention strategies. The improvement model and Plan-Do-Study-Act cycles enabled testing of changes on small scale in specific pediatric clinics/ regions before system-level implementation. Geisinger's pediatric network lead screening completion rate was 49\% in Medicaid patients in 2019 (national Healthcare Effectiveness Data and Information Set rate of 70\%). Initial data showed substantial variation in percentages of both children tested for BLL and with confirmed elevated BLL across sites. As the program is in early development phases, key performance indicator data will be forthcoming.

Conclusion: Multidisciplinary team involvement and system process changes can create pathways to address adherence to complex lead screening guidelines in an integrated health system. During the COVID-19 pandemic, it is important that health systems utilize diverse strategies to prioritize lead testing, especially for children who missed routine BLL screening, are at high risk for lead exposure, and have prior elevated levels needing follow-up testing and intervention.
Trends in Human Papillomavirus Vaccination Among Insured Adolescents in Georgia

Courtney McCracken, Natalie Hernandez, Belkis Pimentel, Ianthia Smith, Hsien-lin Hsieh, Teaniese Davis

Kaiser Permanente Georgia, Atlanta, GA

Background: The human papillomavirus (HPV) is the most common sexually transmitted infection in the United States and has been linked to several forms of cancer. Fortunately, the majority of HPV-related cancers are preventable through vaccination. Despite the safety of HPV vaccination, HPV vaccination rates are lower than many other adolescent vaccines. The goal of this study was to evaluate trends in HPV vaccination among insured adolescents $11-17$ years of age and to identify characteristics associated with HPV vaccination.

Methods: We performed a retrospective cohort study of 11-17-year-old adolescents who were insured by Kaiser Permanente Georgia (KPGA) between January 1, 2012, and December 31,2019. Patient demographics and medical history were extracted from KPGA's virtual data warehouse. HPV vaccination information was ascertained from medical records, claims, and state immunization registry records. HPV vaccination coverage was defined as the proportion of adolescents that had more than $1 \mathrm{HPV}$ dose prior to or during a given calendar year. HPV vaccine completion was defined as receipt of either 2 or 3 doses based on the age of the adolescent and the year vaccination was initiated. Trends over time were examined using the Cochran-Armitage test for trend, and rates were compared between demographic subgroups using chi-squared tests.

Results: There were a total of 74,488 adolescents during the study period. HPV vaccine coverage increased over time $(\mathrm{P}<0.001)$ from $40 \%$ in 2012 to $61 \%$ in 2019 , while completion rates only modestly increased from $31 \%$ to $41 \%$ $(\mathrm{P}<0.001)$. HPV vaccination coverage was slightly higher among females compared to males; however, there was a significant decrease in the gender gap over time $(\mathrm{P}<0.001)$. Asians and African Americans had the highest coverage rates (66\% and $61 \%$, respectively), while Whites had the lowest $(52 \%, \mathrm{P}<0.001)$. Prior flu vaccination was strongly associated with HPV vaccine coverage ( $74 \%$ vs $37 \%$; $\mathrm{P}<0.001$ ).

Conclusion: While HPV vaccination rates have risen over time, vaccination rates still fall well below the Healthy People 2020 benchmark of $80 \%$. These findings suggest the need for continued efforts and interventions to improve HPV vaccination rates and series completion among adolescents. 


\section{MENTAL HEALTH}

\section{Developing a Prospective Cohort of Prescription Opioid Users: Challenges and Lessons Learned}

\author{
Jeffrey Scherrer, Lisa Matero, Scott Secrest, Kirsti \\ Autio, Joanne Salas, Lauren Wilson, Mark Sullivan, \\ Lynn DeBar, Brian Ahmedani, Patrick Lustman
}

\section{Saint Louis University / SSM Health, St. Louis, MO}

Background: Opioid analgesic use (OAU) is associated with an increased risk for depression even after controlling for the contributions from pain. Herein, we describe a research collaboration between Saint Louis University (SLU) and Henry Ford Health System (HFHS) that aims to determine the risk factors, either preceding or following $\mathrm{OAU}$, that increase risk for incident depression and to systematically characterize the depression profile (ie, major depressive episode, dysthymia, bipolar, anhedonia, and vital exhaustion) associated with OAU.

Methods: This is a prospective cohort study of 1500 (750 SLU, 750 HFHS) adult (age range: $18-70$ years) prescription opioid users invited to participate when they have completed a new period (no OAU in previous 3 months) of 30day OAU and have prescriptions allowing for 60 days of continuous opioid use. Targeted subject recruitment using electronic health record data is being used to identify eligible subjects. Baseline, 6-month, and 12-month web or telephone survey are being used to obtain data on lifetime and current psychiatric diagnoses, trauma, sleep disorder, social support, noncancer pain, and OAU. Subjects will be asked to complete 12 monthly assessments measuring painrelated functioning, depression symptoms, and OAU.

Results: Efforts to reduce morbidity and mortality associated with the opioid epidemic have led to significant declines in the number and duration of new opioid prescriptions written in the United States. These changes have challenged our ability to identify eligible subjects and required adapting the eligibility criteria to reflect current prescribing patterns. We now allow tramadol to count as an eligible opioid and do not require a chronic pain diagnosis. We have completed programming of a diagnostic interview in REDCap, making it the first available web-based, lay-administered, psychiatric diagnostic survey. Approximately 500 participants have been enrolled since November 2019.

Conclusion: Restrictions on type of opioid and duration of new opioid prescriptions have dramatically increased the difficulty involved in obtaining eligible patients. Fewer eligible subjects per week has led to allowing for a 24-month baseline enrollment period. Allowing tramadol to count toward OAU greatly increased the number of subjects eligible for study and ensures that results will be relevant to current prescribing practices. Preliminary recruitment information and lessons learned will be discussed.
Increasing Nurse Satisfaction While Decreasing Compassion Fatigue: A Quality Improvement Pilot Project

Alexandra Myers, Ashleigh Boyd

Treat The Nurse, St. Louis, MO

Background: The purpose of this quality improvement project was to identify and reduce the level of compassion fatigue in emergency department (ED) nurses by implementing a self-guided intervention. Compassion fatigue was the largest concern for nurse vacancy rates by administration, and it was confirmed that ED nurses were in the moderate-risk category for compassion fatigue via Professional Quality of Life (ProQOL-5) survey results.

Methods: A Plan-Do-Study-Act method of quality improvement was used for this project. The self-guided intervention of Real-Time Transformative Response ${ }^{\mathcal{O}}$ (RTR) was used. ProQOL-5 was used to measure and determine if it was effective in reducing levels of compassion fatigue in ED nurses.

Results: A total of 34 ED nurses completed RTR training and ProQOL-5 surveys. Overall mean (standard deviation) score was 107 (1.1385) for the pre-RTR training survey and $106.613(1.1677)$ for the post-RTR training survey $(t=1.6924, \mathrm{df}=66 ; \mathrm{P}=0.0476)$. Mean scores pre-and postRTR training for compassion satisfaction increased from 3.59 (0.9521) to 3.95 (0.7766) $(t=1.997, \mathrm{df}=66 ; \mathrm{P}<0.0001)$, for burnout decreased from 2.71 (1.1501) to $2.36(0.9120)$ $(t=1.997, \mathrm{df}=66 ; \mathrm{P}<0.0001)$, and for secondary traumatic stress decreased from 2.45 (1.0002) to 2.25 (0.9143) $(t=1.997, \mathrm{df}=66 ; \mathrm{P}=0.0017)$.

Conclusion: Increasing compassion satisfaction and having an overall decrease in compassion fatigue, burnout, and secondary traumatic stress from using the self-guided RTR methods concluded that it is a valid coping mechanism for nurses.

Patient Perspectives on Acceptability of, and Implementation Preferences for, Use of Electronic Health Records and Machine Learning to Identify Suicide Risk

\section{Bobbi Jo Yarborough, Scott Stumbo}

\section{Kaiser Permanente Northwest, Portland, OR}

Background: Suicide rates in the United States have steadily increased over the past two decades. With recognition that traditional clinical assessments of suicide risk perform only slightly better than chance, attention has turned to the promise of identifying at-risk patients using existing electronic health record data and automated machine learning methods. However, suicide risk identification, being a relatively new application of this technology, lacks implementation 
guidelines or practice standards. While health systems are increasingly interested in use of this technology, we know very little about how patients feel about its use.

Methods: Focus groups informed a web-based survey sent to 11,486 Kaiser Permanente Northwest members in April 2020. Likert-type and bipolar visual analog scales (means of the latter scored -50 to 50) assessed patient understanding of, potential concerns with, and implementation preferences related to automated suicide risk identification using electronic health record data and machine learning. Descriptive statistics summarized findings.

Results: Overall, 1357 (12\%) participants responded to the survey. Most (84\%) found machine learning-derived suicide risk identification an acceptable use of electronic health record data; however, $67 \%$ objected to use of externally sourced data. Participants felt consent (or opt-out) should be required (mean: -14). The majority (69\%) supported outreach to at-risk individuals by a trusted clinician through care messages $(57 \%)$ or telephone calls $(47 \%-54 \%)$. Highest endorsements were for psychiatrists/therapists $(99 \%)$ or a primary care clinician $(75 \%-96 \%)$; less than half (42\%) supported outreach by any clinician, and participants generally felt only trusted clinicians should have access to risk information (mean: -16).

Conclusion: We found patient support for use of electronic health record data (but not externally sourced risk information) to inform automated suicide risk identification models. A patient-centered approach to implementation of these models would involve the option to consent or optout of inclusion and telephone or care message outreach to individuals identified at risk by patients' trusted clinicians.

\section{A Stakeholder-Informed Ethical Framework to Guide Implementation of Suicide Risk Identification Models Using Machine Learning Applied to Electronic Health Records}

\section{Bobbi Jo Yarborough, Scott Stumbo, Gregory Simon}

\section{Kaiser Permanente Northwest, Portland, OR}

Background: Given the pressing need for adequate prevention efforts to arrest the escalating suicide rate in the United States, there has been an urgent focus on suicide risk detection using novel methods including machine learning applied to electronic health records (EHR). Relative to clinical assessment, machine learning algorithms have demonstrated superior accuracy in identifying patients at risk for suicide. Consequently, interest in their use in health care has expanded. A growing body of literature calls for an anticipatory examination of ethical concerns relevant to risk model implementation. In light of active implementation already occurring, and increasing interest in further deployment of suicide risk identification models, there is an urgent need for a practical resource focused on the ethical application of these models in health care delivery settings. Methods: In this multimethods study, patient advisors, a bioethics consultant, and patients and family members participating in formative focus groups (4 focus groups, $n=23$ participants) collectively informed the development of a web-based survey; survey results ( $n=1357$ respondents) and themes from interviews with key informants were used to draft a stakeholder-informed ethical framework to provide practical guidance to health systems considering implementation of suicide risk identification models.

Results: Clinical, ethical, operational, and technical issues reiterated by multiple stakeholder groups and corresponding questions for risk identification model adopters to consider prior to and during suicide risk identification model implementation are organized within 6 ethical principles in the resulting stakeholder-informed framework.

Conclusion: Enthusiasm for machine learning in the context of suicide is understandable given the significance of the suicide epidemic in the United States. Attention to ethical and practical concerns in advance of automated suicide risk identification model implementation may help avoid unnecessary harms that could thwart the promise of this innovation in suicide prevention. An ethical framework can help to anticipate and reduce potential for harms, keeping the safety and welfare of the patients and their trust at the center of health care decision-making.

\section{Universal Screening for Suicidal Thoughts and Behaviors in a Large Health System's Hospital and Emergency Departments}

Ellis C. Dillon, Kristen M.J. Azar, Ernell De Vera, Martina Li, Qiwen Huang, Sien Deng, Anna Kiger, Daniel Becker, Tam Nguyen, Jacqui Pesa, Jennifer Voelker, Giacomo Salvadore

\section{Sutter Health, Palo Alto, CA}

Background: Suicide is the $10^{\text {th }}$-leading cause of death in the United States. The Joint Commission recently revised its National Patient Safety Goal requirements to include standardizing suicide screening. In response, Sutter Health, a large system serving 3 million patients in Northern California, launched systemwide standardized use of the Columbia Suicide Severity Rating Scale (C-SSRS) in acute care facilities in July 2019. Our goal was to describe and compare characteristics of adult patients screening positive for suicidal thoughts or behaviors (STB), including those with major depressive disorder (MDD).

Methods: Electronic health record-based observational cohort study describing and comparing sociodemographic and clinical characteristics of adult patients screening positive for STB (July 1, 2019-October 4, 2020), including those with MDD. Patients with STB and MDD (STB+MDD) were compared to those with STB without 
MDD (STB-MDD) and on risk categories (no risk vs any risk). Wilcoxon rank-sum tests for continuous variables and $\chi^{2}$ tests for categorical variables were used. Results reported are statistically significant at 0.05 .

Results: Of 478,143 adults identified, 410,302 (85.8\%) were screened by C-SSRS. Among those screened, 8416 (2.05\%) had STB; in that group, 914 (10.9\%) were STB+MDD and 7502 (89.1\%) were STB-MDD. Of those with STB, 3278 (38.9\%) were low risk, 1314 (15.6\%) moderate risk, and 3824 (45.4\%) high risk. Any risk vs no risk comparisons by characteristic were: male gender $(46.6 \%$ vs $42.3 \%)$, White race (55.5\% vs $47.9 \%)$, mean age (41.6 vs 50.3 years), homeless in last 12 months (12.0\% vs $2.5 \%$ ), index encounter in psych unit ( $10.3 \%$ vs $0.2 \%)$, mental health diagnoses $(22.2 \%$ vs $4.9 \%)$, and MDD (10.9\% vs 1\%). STB+MDD patients vs STB-MDD comparisons by characteristic were: mean age (38.8 vs 41.9 years), female gender (58.6\% vs $52.8 \%$ ), Asian race $(9.7 \%$ vs $6.6 \%)$, income of $\$ 75,000+(45.5 \%$ vs $34.0 \%)$, index encounter in psych units (46.5\% vs 5.9\%), substance abuse (12.1\% vs $3.9 \%)$, anxiety (16\% vs $2.2 \%)$, and documented prior suicidal ideation $(3.7 \%$ vs $0.5 \%)$.

Conclusion: Screening for STB identified 2.05\% with any STB, $10.9 \%$ of which were STB+MDD. Characteristics differed based on risk and MDD status. Highlighting demographic and clinical differences between these patient groups can guide efforts to provide mental health consultation and referrals. Future research may establish if universal screening in acute care settings leads to improved follow-up care for those with STB.

\section{Weight Loss Improves Mental Health Status in a Behavioral Weight Loss Trial}

Cody Goessl, Jeff VanWormer, Ram Pathak, Ed Ellerbeck, Christie Befort

\section{Marshfield Clinic, Marshfield, WI}

Background: Obesity, depression, and anxiety often cooccur, but there is limited prior research on weight change and mental health status. The purpose of this study was to examine how Short-Form 12 Health Survey mental health (SF-12MH) scores changed over 24 months between obese adult trial participants with vs without a baseline mental condition, as well as those who lost or gained weight.

Methods: There were 1407 participants enrolled in a 3-arm behavioral weight loss trial based in rural primary care practices across Kansas, Nebraska, and Wisconsin. All participants received a lifestyle intervention with different delivery models, including in-clinic individual, in-clinic group, or telephone group counseling visits. In this analysis, the 1148 participants with complete data were stratified by those with vs without a baseline mental health condition (receiving medication or counseling for depression, anxiety, or adjustment issues), as well as by 24 -month weight-change quintiles. Mixed models were used to estimate SF-12MH scores by mental health condition and weight-change categories.

Results: There was a significant group-by-time interaction at 24-month follow-up. The largest 0-24-month increase in SF-12MH scores $(+5.3$ points $[12 \%])$ was observed in participants with a baseline mental health condition who lost the most weight (quintile) during the trial, while the largest decrease in SF-12MH scores (-1.8 points [-3\%]) was observed in participants without a baseline mental health condition who gained the most weight $(\mathrm{P}<0.001)$.

Conclusion: Mental health status modestly improved in this trial with obese adults but particularly increased among participants with a baseline mental health condition who experienced significant weight loss. Those without baseline mental health concerns who gained weight, however, actually experienced a decline in mental health status over 24 months. Although the causal direction cannot be firmly established given our analytical design, study findings support the mental health benefits of weight loss in obese adults with depression or anxiety. They also suggest that failure to achieve weight loss could be psychologically consequential for at least some participants.

\section{Medication Patterns for Patients With Depression, Anxiety, and Sleep Disorders}

Jennifer M. Boggs, Erica Morse, Kathleen Albers, Arne Beck

\section{Kaiser Permanente Colorado, Denver, CO}

Background: Depression, anxiety, and sleep disorders are the most common mental health disorders and highly comorbid. Despite a wide range of pharmacological treatments informed by numerous randomized clinical trials, real-world medication patterns to treat these comorbidities involve significant adaptations. Treatment is further complicated by safety concerns for benzodiazepines, including addiction, falls, and dementia. We used a mixed-methods approach to understand real-world prescribing patterns and to identify relevant research questions for future study.

Methods: Kaiser Permanente Colorado patients were identified with new medication treatment episodes (4-month washout) initiated by dispensing of antidepressants (selective serotonin reuptake inhibitor, serotonin-norepinephrine reuptake inhibitor, norepinephrine-dopamine reuptake inhibitors, tricyclic), benzodiazepines, z-drug, or atypical antipsychotics. Patients had at least one comorbidity for anxiety, depression, or sleep within a 1-year period (20092018) and no bipolar, psychosis, dementia, or attentional disorders. Dispensing data (from Virtual Data Warehouse) were evaluated to determine frequencies of the following medication patterns: stabilization (no changes for 90 days), medication changes (stopping one, starting another), stopping (all medications stopped for 60 days), and co-prescribing. 
Results: A total of 56,862 patients were prescribed medications by primary care providers (PCP) or psychiatrists, with $11 \%$ receiving prescriptions from the latter. More than half $(57 \%)$ reached stabilization, with an average of 1.1 changes before stabilization. Patients treated by psychiatric providers were more likely to reach stabilization $(70 \%$ vs $55 \%)$. Those who stopped all medications were less likely to have medication changes (average number of changes: 0.5 ). Nine providers (6 PCP, 3 psychiatry) were interviewed; all indicated they rarely prescribed benzodiazepines, but one-third of their patients received them. All providers discussed challenges with managing patient expectations for effectiveness and medication side effects with variation in counseling strategies. Providers indicated having a handful of patients they "couldn't get off" benzodiazepines, which was observed in the electronic health record data. Approximately $8 \%$ of patients who started a benzodiazepine remained on it beyond 90 days, indicating likely physical dependence.

Conclusion: These findings showed several important areas for future research on comorbid depression, anxiety, and sleep disorders: 1) understanding risk factors for benzodiazepine dependence; 2) differences in treatment patterns between psychiatric and nonpsychiatric providers; and 3) counseling strategies that emphasize the importance of trial and error with medication switching to achieve stabilization.

\section{Understanding the Role of Mental Health and Substance Abuse in Perpetrating Violent Acts: In the Presence of Unmet Needs for Mental Health Services}

Ruaa Al Juboori, Divya Subramaniam, Leslie Hinyard

\section{Saint Louis University / SSM Health, St. Louis, MO}

Background: Less is known about how the association between substance use, mental health, and violent acts might vary depending on perceptions of unmet needs for mental health services. This study aimed to explore the association between unmet need for mental health services, health insurance, substance abuse, and mental health problems and the perpetration of violent acts. Its second aim was to determine if unmet need for mental health services is an effect modifier in the relationship between predictors and violence perpetration.

Methods: Year 2019 data from adults $(\geq 18$ years old) in the National Survey on Drug Use and Health $(n=42,567)$ was used. Logistic regression was conducted to examine the relationship between attacks intended to seriously hurt others and unmet needs for mental health services, health insurance, major depressive episode (MDE), suicidal ideation, and substance abuse. Interaction terms between unmet needs for mental health services and predictors were created to test for the effect-modifying role of unmet needs for mental health services between predictors and outcome.
Results: In this adult population, $469(1.1 \%)$ reported attacking someone with the intent to seriously hurt in the last 12 months. Unmet needs for mental health services, suicidal ideation, MDE, alcohol, and other illicit drug abuse were associated with higher likelihood of violence perpetration. The interaction terms showed that in the presence of unmet need for mental health services, there were amplified associations for suicidal ideation (adjusted odds ratio [aOR]: 5.34, 95\% CI: 4.10, 6.59) MDE (aOR: 1.95, 95\% CI: 1.46 , 2.60), and alcohol abuse (aOR: $3.10,95 \%$ CI: $2.49,3.85$ ) with violence perpetration.

Conclusion: Study results highlight the importance of providing mental health coverage for individuals experiencing substance abuse and mental health disorders in order to prevent violence. Therefore, it is valuable to have mental health experts highly involved in violence risk assessment among patients with mental health and substance abuse disorders. Yet, prospective studies are needed to assess causal pathways.

\section{Health Service Utilization Among Children and Adolescents With Posttraumatic Stress Disorder: A Case-Control Study}

Frances Lynch, Pauline Goger, Argero Zerr, John Dickerson, Phillip Crawford, Stacy Sterling, Yihe Daida, Brian Ahmedani, Robert Penfold, Robin Weersing

\section{Kaiser Permanente Northwest, Portland, OR}

Background: Trauma exposure is widely prevalent, with over $60 \%$ of adolescents having experienced at least one traumatic event and one-third of those at high risk to develop posttraumatic stress disorder (PTSD). Data on the services youths with PTSD receive are scarce and out of date, impeding efforts to improve care and outcomes. This study examines health services use for a large and diverse sample of youths with and without a diagnosis of PTSD.

Methods: Utilizing a matched case-control study, we gathered information from 4 large health systems participating in the Mental Health Research Network (MHRN): Kaiser Permanente Northwest; Henry Ford Health System; Kaiser Permanente Northern California; and Kaiser Permanente Hawaii. Data from each site's electronic medical records and claims data on diagnoses, encounters, and demographics were analyzed. A total of 955 youths (age range: 4-18 years) with a diagnosis of PTSD were identified and matched on an approximately $1: 5$ ratio to 4770 controls. We compared cases to controls on frequency of service use in primary care, medical specialty care, acute care, and in mental health care. We also assessed psychotropic medication use.

Results: Youths diagnosed with PTSD utilized care in nearly all physical and mental health service categories at a higher rate than control youths. In particular, youth with PTSD are more likely to use outpatient mental health (odds ratio: 9.09; $\mathrm{P}<0.001$ ) and psychotropic medication (odds ratio: 6.56; 
$\mathrm{P}<0.001)$. However, one-third of youths did not receive even one outpatient mental health visit $(36.86 \%)$ during the yearlong observation window.

Conclusion: Only about two-thirds of youth with PTSD received outpatient mental health services, while half received medications. Per practice parameters for youth with PTSD, psychotropic medications such as selective serotonin reuptake inhibitors can be an important adjunct to psychotherapy, especially for youth with comorbid conditions or more severe symptoms. Thus, this rate of medication dispensing may indicate unmet need. Most critically, more than one-third of youth diagnosed with PTSD did not receive any mental health services at all during the study period. Youths with PTSD are high utilizers of health services overall but lower utilizers of the treatment sectors that may be most helpful in resolving their symptoms.

\section{Health Diagnoses and Service Utilization in the Year Prior to Youth and Young Adult Suicide}

Jordan Braciszewski, Ana Lanier, Hsueh-Han Yeh, Kelsey Sala-Hamrick, Gregory Simon, Rebecca Rossom, Frances Lynch, Stephen Waring, Christine Y. Lu, Ashli Owen-Smith, Arne Beck, Beth Waitzfelder, Melissa Maye, Cathrine Frank, Melissa Hendriks, Nina Fabian, Brian Ahmedani

\section{Henry Ford Health System, Detroit, MI}

Background: Suicide rates among adolescents have risen steadily since 2007 , creating a dire need to expand prevention protocols. Health care visits have been identified as key opportunities for identification and intervention. To date, studies of youth and young adult health conditions and service utilization have been limited by small or circumscribed samples.

Methods: A case-control study was conducted using records from 9 health systems nationwide. Data from 450 members, 10-24 years of age, who died by suicide between the years 2000 and 2015 were matched with data from 4500 controls based on health system and time period of membership. We examined past-year recorded diagnoses, patterns of visit types, and frequency of those visits.

Results: Adolescents who died by suicide were more likely to have at least one recorded mental health diagnosis $(51 \%$ vs $16 \%$; adjusted odds ratio: $5.74,95 \% \mathrm{CI}: 4.60-7.18)$ and higher rates of each individual mental health condition except for autism. Adjusting for age and gender, odds ratios ranged from 2.85 (ADHD) to more than 16 (eating disorders, other psychoses). Recorded diagnoses of brain injuries, epilepsy, and sleep disorders also were more likely among cases. Close to half (49\%) and nearly all (89\%) youth who died by suicide had a health care visit in the month and year prior to their death, respectively. Across all visit types and time frames (30, 90, and 365 days), cases were more likely to visit the health system than controls. Visits for outpatient specialty care were most common in the past year, with suicide decedents making an average of 8 visits in the year before death, only 4 of which were associated with a recorded mental health diagnosis.

Conclusion: With nearly half (49\%) of adolescents who died by suicide lacking a recorded mental health diagnosis in the year prior to their death, relying only on mental health services to identify at-risk adolescents may not be sufficient. High rates of health care utilization among those who died by suicide indicate a strong need for improving identification of youth while they are seeking services, thereby preventing future deaths.

\section{Demographic and Psychosocial Factors Associated With Suicide Mortality Among Childbearing-Age Individuals: A Case-Control Study}

Leah Hecht, Amy M. Loree, Hsueh-Han Yeh, Lyubov Gavrilova, Katerina Furman, Joslyn Westphal, Gregory Simon, Frances Lynch, Arne Beck, Ashli Owen-Smith, Rebecca Rossom, Yihe G. Daida, Christine Y. Lu, Jennifer M. Boggs, Cathrine Frank, Stephen Waring, Brian Ahmedani

Henry Ford Health System, Detroit, MI

Background: Depression is common among individuals of childbearing age, particularly during the perinatal period. Although suicide rates tend to be lower among those who are pregnant, potential risk factors of maternal suicide need to be better understood. The goal of this study was to examine demographic and psychosocial factors, including pregnancy and postpartum status and perinatal loss (eg, stillbirth, abortion) associated with suicide among a large diverse sample of childbearing-age individuals.

Methods: The study sample was obtained from 9 health systems in the Mental Health Research Network. This study used a case-control study design, with 290 childbearing-age individuals who died by suicide (cases) during 2000-2015 each matched to 10 childbearing-age individuals from the same health system and enrolled during the same time period who did not die by suicide $(\mathrm{n}=2900)$. Conditional logistic regression was used to investigate the relationship between pregnancy/postpartum status and suicide death in this matched case-control study.

Results: Childbearing-age individuals who died by suicide were more likely to have mental health disorder diagnoses including substance use disorders (adjusted odds ratio [aOR]: $2.69,95 \%$ CI: $1.69,4.26)$ and more likely to have visited the emergency department in the year prior to index date (ie, date of suicide death) (aOR: 3.44, 95\% CI: 2.50, 4.73). Childbearing-age individuals who were pregnant (aOR: 0.14 , 95\% CI: $0.03,0.63$ ) or gave birth (aOR: $0.38,95 \%$ CI: 0.16 , 0.87 within a year before the index date had a lower risk 
of dying by suicide compared to individuals who had not experienced any pregnancy events. However, individuals who experienced perinatal loss were more likely to die by suicide (aOR: $1.43,95 \%$ CI: 0.51, 4.00), although due to the small number of patients with this event $(n=6$ cases, $n=21$ control), the result was not statistically significant.

Conclusion: While pregnancy and delivery may be protective against suicide, perinatal loss may be associated with higher suicide rate. Routine screening and monitoring for anxiety, depression, substance use disorders, and prior emergency department encounters among individuals who experience pregnancy loss is warranted given their increased risk for suicide. Future studies should further examine associations between type of perinatal loss (eg, early infant death, miscarriage, abortion, stillbirth) and suicide mortality.

\section{Visual and Ocular Phenotypes Associated With Autism and Atypical Neurodevelopment Using Electronic Health Records}

Antoinette DiCriscio, Jaclyn Smith, Julie-Anne Little, Anthony Seiwell, Brian Harry, Vanessa Troiani

\section{Geisinger Research, Lewisburg, PA}

Background: Atypical visual perception, such as "seeing the trees instead of the forest," has been frequently documented in autism spectrum disorder (ASD) and is generally attributed to atypical brain function. However, relatively little research has characterized optometric anomalies in ASD, which may indicate atypical morphology and/or malfunction of the eye rather than the brain. Increased prevalence of refractive errors and optic nerve abnormalities in ASD have been found in studies with small sample sizes, but this work has yet to be completed at a large scale. The current study leverages existing optometry and ophthalmologic records to establish visual and ocular phenotypes in patients with and without known genetic causes of ASD and atypical neurodevelopment.

Methods: We evaluated the presence of atypical ocular features via manual chart review of electronic health record data in 563 patients (age range: 3-21 years), including ophthalmologist/optometrist visits across 3 groups: $n=210$ neurodevelopmental probands with a genetic syndrome associated with ASD; $n=156$ with ASD diagnoses with no known genetic cause; and n=197 age- and sex-matched controls. We characterized structural and functional ocular symptoms using a rubric based on the presence or absence of symptoms across 6 domains: 1) visual acuity; 2) refractive error, accommodative disorders, and near visual function; 3) eye movements; 4) stereopsis and oculomotor function; 5) retinal function and structure; and 6) photoresponse, photosensitivity, and pupil response.

Results: We found increased documentation of visual and ocular symptoms in neurodevelopmental probands and ASD with no genetic cause as compared to healthy controls, including features of abnormal eye movements $(\mathrm{P}<0.0254$ for all comparisons), amblyopia ( $\mathrm{P}<0.0367$ for all), strabismus $(\mathrm{P}<0.0037$ for all) and esotropia ( $\mathrm{P}<0.0032$ for all). We also found increased prevalence of astigmatism and atypical accommodation in neurodevelopmental probands as compared to both ASD and healthy controls ( $\mathrm{P}<0.0140$ for all).

Conclusion: Results indicate that ocular and ophthalmologic anomalies are more frequently present in children with neurodevelopmental differences compared to controls. These results highlight the feasibility of retrospective chart review to curate ocular variables acquired as part of standard clinical care, indicate a potential path for intervention in neurodevelopmental disorders, and lay the groundwork for new analyses that explore the genetic underpinnings of ocular anomalies within ASD subtypes.

\section{Trauma Diagnoses and Risk for Suicide Mortality in a Health System Patient Population}

Kelsey Sala-Hamrick, Jordan Braciszewski, Rachel Zelenak, Joslyn Westphal, Cathrine Frank, HsuehHan Yeh, Gregory Simon, Ashli Owen-Smith, Rebecca Rossom, Frances Lynch, Christine Y. Lu, Stephen Waring, Arne Beck, Yihe Daida, Brian Ahmedani

\section{Henry Ford Health System, Detroit, MI}

Background: Strong evidence exists for posttraumatic stress disorder (PTSD) as a risk factor for suicidal thoughts and behaviors. PTSD also has been identified as a serious risk factor for suicide mortality in U.S. veteran populations and in a Danish epidemiological sample. However, limited empirical studies examine PTSD and other trauma disorders as risk factors for suicide mortality, particularly among non-veteran health system patients in the United States. Additionally, this study further contributes to the field by considering a wider spectrum of trauma conditions (not isolated to the single diagnosis of PTSD) as risk factors for suicide mortality by including other trauma- and stressrelated diagnostic conditions (ie, acute stress disorder and other reactions to stress disorders [ICD-9 diagnostic codes 309.89, 309.9, 309.0-4, 309.82, and 309.83]).

Methods: Using a case-control matched design, this study uses a dataset consisting of health plan-affiliated patients who died by suicide between 2000 and 2015 from 9 different U.S. health systems through participation in the Mental Health Research Network. Patients with PTSD ( $n=120)$, acute stress disorder $(\mathrm{n}=84)$, and other reactions to stress disorders $(\mathrm{n}=331)$ who died by suicide were case-matched by time period and site to a sample of controls $(\mathrm{n}=1592$, $\mathrm{n}=2218$, and $\mathrm{n}=8174$, respectively). Patients were identified as having a trauma condition utilizing ICD-9 diagnostic codes (recorded in the electronic medical records and/or insurance claims data) at all participating health systems. 
Results: Adjusting for age and gender, individuals with diagnosed PTSD were more likely to die by suicide $(3.6 \%$ vs $0.5 \%$; adjusted odds ratio [aOR]: $10.10,95 \%$ CI: $8.31-$ 12.27). Patients with diagnosed acute stress disorder $(2.5 \%$ vs $0.7 \%$; aOR: $4.49,95 \%$ CI: $3.58-5.62)$ and those diagnosed with other stress reactions ( $9.9 \%$ vs $2.4 \%$; aOR: $5.38,95 \%$ CI: 4.78-6.06) also were more likely to die by suicide.

Conclusion: This study suggests that both PTSD and other trauma disorders in health system patient populations are serious risk factors for suicide mortality. These findings highlight the importance of population-level screening (ie, across health systems) to identify individuals with trauma diagnoses as well as providing and expanding traumainformed mental health services in a suicide prevention strategy.

\section{A Segmented Individual Trajectory Analysis for Identifying Opioid Use Patterns and Examining Their Association With Opioid Overdose}

Stanley Xu, Komal Narwaney, Morgan Ford, Jason Glanz, Ingrid Binswanger

\section{Kaiser Permanente Southern California, Pasadena, CA}

Background: Understanding how to adjust opioid dose in patients on long-term opioid therapy (LTOT) for pain while minimizing risk of unintentional harm is critical in combating the opioid crisis. Researchers have assessed the safety of limited opioid use patterns in chronic pain populations using electronic health record (EHR) data in observational studies. However, existing methods have limitations. The objective of this study was to use electronic opioid-dispensing data to develop a segmented individual trajectory analysis for identifying opioid use patterns and evaluating their association with opioid overdose.

Methods: Daily opioid doses in morphine milligram equivalents (MMEs) averaged over a 30-day period were calculated for a cohort of adult members on LTOT in an integrated health system between January 1, 2006, and December 31, 2019. An individual's follow-up started upon LTOT eligibility and ended at either disenrollment, first overdose, death, or the end of the study, whichever occurred first. For each individual, we used variable scan windows and coefficient of variation to find periods with stable and nonstable MMEs. We then categorized these periods into 11 patterns, including relatively common patterns such as "discontinued," "stable," "slow tapering," "fast tapering," and "increasing" periods. Incidence rates of opioid overdose were calculated for these relatively common patterns.

Results: The study population $(\mathrm{N}=18,658)$ had 92,350 personyears of follow-up, including $59.0 \%$ in stable periods, $16.9 \%$ in discontinued periods, $9.8 \%$ in increasing periods, $8.1 \%$ in slow tapering periods, and $4.2 \%$ in fast tapering periods. Incidence rates of overdose per 1000 person-years were
$0.13,0.23,0.17,0.77$, and 0.59 for discontinued, stable, slow tapering, fast tapering, and increasing periods, respectively.

Conclusion: We developed a novel approach that identified 11 mutually exclusive opioid use patterns and showed that "fast tapering" had the greatest incidence rate of opioid overdose among relatively common patterns.

\section{METHODS, DESIGN, AND ANALYTIC TOOLS}

\section{Assessing the Feasibility of Collecting a Core Set of Harmonized Depression Outcome Measures in Multiple Real-World Care Settings}

Michelle Leavy, Diana E. Clarke, Debbie Gibson, Sarah Hajjar, Eric Bickelman, Richard Gliklich

OM1, Inc., Boston, MA

Background: Major depressive disorder is a significant public health problem in the United States, and new research is needed to improve diagnosis, treatment, and patient outcomes. Real-world data sources, such as electronic health records and patient registries, offer the potential for efficient, large-scale studies, but variations in the data collected across real-world care settings make it challenging to use these data for new research studies. The purpose of this effort was to assess the feasibility of collecting a core set of harmonized depression outcome measures in multiple real-world care settings.

Methods: The Agency for Healthcare Research and Quality (AHRQ) recently supported an effort to harmonize depression outcome measures across patient registries and clinical practice. Implementation of these measures in patient registries and in the clinical practice setting would support both routine clinical care, specifically measurement-based care using the PHQ-9, and development of data infrastructure to address new depression-related research questions. In this AHRQ-funded pilot study, we implemented harmonized measures (remission, response, recurrence, suicide ideation and behavior, adverse events related to treatment) in 2 patient registries (the PRIME Registry sponsored by the American Board of Family Medicine and the PsychPRO sponsored by the American Psychiatric Association) and 1 health system (Baystate Health).

Results: Calculation of the harmonized depression measures was feasible in the primary care and mental health care settings. Technical and operational efforts are necessary to ensure that the data are recorded using structured fields in medical records, as opposed to in clinical notes. Also, low patient response rates impacted collection of the PHQ-9 outside of an office visit, due in part to the implementation curve associated with measurement-based care as well as the data collection occurring during the COVID-19 pandemic. 
Conclusion: While many efforts have developed harmonized or core sets of outcome measures, few efforts have undertaken pilot studies to examine the impact and challenges of implementing the measures. This project provides important information on potential barriers to help inform other implementation efforts.

\section{Constructing a Severity Index for Knee Osteoarthritis for Use in Administrative Datasets}

\author{
Sarah Gebauer, Tim Chrusciel, Joanne Salas, Jeffrey \\ Scherrer
}

\section{Saint Louis University / SSM Health, St. Louis, MO}

Background: Osteoarthritis is one of the most common disabling conditions worldwide. Big Data approaches offer a wealth of information to explore the clinical trajectory of osteoarthritis and potential targets for intervention. One must account for osteoarthritis severity because the definitive treatment, total knee arthroplasty (TKA), is often only offered after failure of nonsurgical management. An osteoarthritis severity index (OSI) utilizing electronic health record (EHR) data would allow the quantification of severity without clinical interaction. The aim of this study was to explore methods to develop a novel administrative OSI to predict TKA.

Methods: This retrospective cohort study used a national EHR database of 5 million adults from 2010 to 2018. Eligible patients were 45-80 years old with a new diagnosis of knee osteoarthritis in 2011-2012 and no history of TKA. The outcome was time to TKA. The OSI was a time-dependent variable updated every 6 months in follow-up. Treatments included X-ray, advanced imaging, intra-articular injection, nonsteroidal anti-inflammatory drugs, and opioids. Principal components analysis index score (PCI) and a basic sum were explored as possible OSI indices. Extended Cox proportional hazard models adjusted for time-dependent comorbidities were used to calculate hazard ratios (HR).

Results: Of the 16,675 patients meeting inclusion criteria, $2119(12.7 \%)$ underwent TKA. Follow-up time ranged from 6 to 96 months. The cohort was $44.8 \% 65+$ years old, $63.1 \%$ female, and $82.9 \%$ White. PCI and sum OSI indices both showed that a unit increase doubled the risk for TKA (HR: 2.02, 95\% CI: $1.95-2.10$ vs HR: $1.95,95 \%$ CI: $1.88-2.02$, respectively). Results did not differ substantially between unadjusted and adjusted models (PCI unadjusted HR: 2.00, 95\% CI: 1.93-2.07; sum OSI unadjusted HR: $1.91,95 \%$ CI: $1.85-1.98)$.

Conclusion: Since PCI and sum OSI showed similar results, using a simpler sum score would perform well at identifying patients who would ultimately undergo TKA. The likelihood of TKA almost doubled with each additional treatment category received. Next steps include replication in another administrative dataset, inclusion of pain score, and application and evaluation of performance in geospatial models.

\section{A Study of the Aging Brain Using Electronic Health Record Brain Imaging}

\section{Donielle Beiler, Arnab Roy, Vanessa Troiani}

\section{Geisinger Research, Lewisburg, PA}

Background: Magnetic resonance imaging (MRI) can be a valuable tool for understanding disease processes of the human brain, and MRI analysis has contributed to our understanding of brain development, aging, and cognitive processing. Studies of the brain at the group level tend to utilize prospective research recruitment strategies, requiring substantial time and money for the acquisition of substantial population cohorts. Conversely, health systems amass large collections of MRIs as part of routine patient care, but clinically ascertained imaging tends to only be used for case or small cohort studies. Herein, we examine the viability of using clinically ascertained MRIs to establish a research cohort and assess whether we can confirm that gray matter volumes diminish with age as an initial experiment using the cohort.

Methods: To establish this population cohort, 12,030 brain MRI sessions from 9236 patients were identified based on having scan sequence names consistent with higher resolution acquisitions (ie, MPRAGE, FSPGR, 3-dimensional SPGR T1). Diagnostic history, prescribed medications, and patient-level variables including age, sex, and vitals recorded at the time of the scan were exported. Patient-level variables and MRIs were deidentified using an approved data broker process, and then MRI data were analyzed using a voxel-based morphology pipeline. As a test experiment using this cohort, we then compared gray matter, white matter, and cerebral spinal fluid volumes between individuals that were either 18-22 years old $(n=181)$ or $65-69$ years old $(n=193)$ at the time of the scan.

Results: As expected, statistically significant differences were detected in gray matter volume between the young and aging cohorts when comparing individuals of the same gender $(\mathrm{P}=1.07 \mathrm{E}-38$ for females; $\mathrm{P}=4.04 \mathrm{E}-26$ for males) but not in white matter or total intracranial volume. We also confirm known differences of total intracranial volume within each cohort across genders $(\mathrm{P}=2.97 \mathrm{E}-10$ for age 18 22 ; $\mathrm{P}=5.93 \mathrm{E}-13$ for age $65-69$ ).

Conclusion: These results suggest that use of clinically obtained, high-resolution MRIs produce results comparable to MRIs obtained via research protocols for global gray matter, white matter, and cerebral spinal fluid volume. This largely untapped resource can be used to access substantial cohorts with significantly less time and cost than traditional prospective cohort recruitment. 


\section{Breathing Life Into Data Using the Lives Impacted Method: A Distinctive Approach to Estimating Health Impact and Enhancing Health Care Performance Data Applicability}

\author{
Alan Bienvenida, Kate Koplan, Felipe Lobelo \\ Kaiser Permanente Georgia, Atlanta, GA
}

Background: Traditional health care performance is not expressed into easy-to-understand outcome-driven measures, and few attempts have been made to translate these indicators into population health impact estimations. Furthermore, previous research studies in this area have relied on complex modeling that may be difficult to replicate by health systems for operational uses and quality improvement initiatives. We developed a valid and replicable method to estimate total cases of morbidity and mortality averted through chronic disease prevention and control services within an integrated health system.

Methods: This tool uses retrospective health plan performance rates provided by the Healthcare Effectiveness Data and Information Set (HEDIS) and number needed to treat/screen published estimates. Patient-level data of HEDIS-eligible Kaiser Permanente Georgia members were used to create an adjustment factor to account for people with multiple chronic conditions. With this approach, we conservatively estimated the crucial population health impact of chronic disease morbidity and mortality averted among our integrated health system membership. We compiled and analyzed 10-year trends (2008 to 2018) in HEDIS for 10 metrics specific to diabetes care, cardiovascular control, and cancer screenings. We accessed both health management organization (HMO) commercial and Medicare product line data for each HEDIS metric. All individuals who met the HEDIS eligibility criteria were included.

Results: For the 2008-2018 period, we conservatively estimate a total of 15,123 lives were crucially impacted by prevention and control services resulting in chronic disease morbidity and mortality averted. Prospectively, we estimate that we can impact 68 to 596 more lives annually in scenarios with membership growth and/or improvements in average HEDIS performance rates in the HMO commercial and Medicare populations.

Conclusion: The Lives Impacted method is a distinctive approach that uses HEDIS data to estimate the impact that a health system can have on the population it serves. The Lives Impacted method can also forecast future estimates of health impact and enable comparisons with other publicly reported quality data. This approach can aid health care organizations to better communicate the crucial impact of their prevention and control services and shape quality-ofcare objectives.
Does Exposure to School Violence Predispose Adolescents to Engage in Risky Sex?

\author{
Ruaa Al Juboori, Divya Subramaniam
}

\section{Saint Louis University / SSM Health, St. Louis, MO}

Background: School-based violence is a public health problem in the United States. While mortality, serious injury, and adverse mental health outcomes are the most severe consequences of violence, if left untreated, violenceexposed adolescents might engage in maladaptive behaviors to cope with their traumatic experience(s). Despite that, many adolescents are not being assessed for school violence exposure and its implications by the educational systems with which they frequently interact. Hence, we investigated the association between school-based violence (being threatened/injured on school property) and having last sex under the influence of alcohol/drugs, in sexually active U.S. high school adolescents.

Methods: Prevalence trend of having last sex under the influence of alcohol/drugs and predictors (1991-2019) were examined. Descriptive and multivariate logistic regression analyses were used. Total sample used in this multivariate analysis was 3637 sexually active adolescents. Regression models were adjusted for demographics (age, sex, race/ ethnicity), early initiation of risky behaviors (smoking, alcohol, and marijuana), mental health indicators (suicidal attempt, depression), and good school performance.

Results: Analysis showed that adolescents who had sex under the influence of alcohol/drugs accounted for $21 \%$ of the sexually active adolescents. Adolescents who had been threatened/injured with a weapon on school property showed higher odds of engagement in having last sex under the influence of alcohol/drug (adjusted odds ratio [aOR]: 1.58, $95 \%$ CI: 1.13, 2.19). Results were significant after adjusting for potential confounders in good school performance (aOR: $0.69,95 \%$ CI: 0.55, 0.58); suicidal attempt (aOR: $1.34,95 \%$ CI: $1.03,1.74)$; and early initiation of the risky behaviors drinking alcohol (aOR: 1.59, 95\% CI: 1.25, 2.20), smoking (aOR: 1.57, 95\% CI: 1.20, 2.06), and marijuana (aOR: 2.36, 95\% CI: 1.79, 3.14).

Conclusion: Findings indicate that violence exposure is associated with risky sexual behaviors. Therefore, targeted multidimensional interventions should be developed to combat school-based violence as a preventative measure for risky sexual behaviors. School counseling services ought to pay attention to adolescents who are at risk or victims of school-based violence. Intervention programs should consider confidential screening for risky sexual behaviors among victims of violence to help provide referrals to prevention services when appropriate. Further, longitudinal research is needed to better understand school-based violence and risky sexual behaviors. 


\section{Changes in Clinician Well-Being and Needs Over Time During the COVID-19 Pandemic and the Increased Burden on Female Clinicians}

Ellis C. Dillon, Cheryl Stults, Sien Deng, Meghan Martinez, Amaka Agodi, Nina Szwerinski, P.T. Koenig, Melissa Hanley, Sarina Le Sieur, Jill Kacher Cobb, Laurie Gregg, Suzanne Pertsch

\section{Sutter Health, Palo Alto, CA}

Background: COVID-19 has dramatically changed clinicians' personal and professional lives. At Sutter Health, the Joy of Work team sought to support clinicians by conducting repeated surveys that were used to ask about well-being, gather actionable information, and identify improvement opportunities.

Methods: We developed 5 core questions about leadership, safety at work, caregiving, what can be done to support clinicians, and burnout (using a validated single-item measure) and asked them on surveys distributed by our research team and by NRC Health in June-August and again in October-December of 2020. A total of 10,916 surveys were distributed in summer and 9318 in fall to clinicians in 8 affiliated medical groups and 17 Sutter hospitals across Northern California.

Results: Overall, $3470(31.8 \%)$ surveys were returned in summer and $4556(48.9 \%)$ in fall. The burnout rate was $30.1 \%$ in summer and $29.0 \%$ in fall. Burnout was more common among women than men (summer: $38.4 \%$ vs $24.6 \%$; $\mathrm{P}<0.001$; fall: $35.1 \%$ vs $21.7 \%$; $\mathrm{P}<0.001$ ). Between summer and fall, the proportion of clinicians concerned about safety decreased ( $42.7 \%$ to $21.7 \% ; \mathrm{P}<0.001)$ and the proportion who believed their concerns would be acted on increased (53.9\% to $60.7 \%$; $\mathrm{P}<0.001$ ). Of participants with caregiving responsibilities, the proportion indicating that childcare/caregiving impacted work was $32.6 \%$ (summer) vs $31.7 \%$ (fall) and more women reported this than men (summer: $42.2 \%$ vs $23.7 \%$; $\mathrm{P}<0.001$; fall: $36.7 \%$ vs $26.0 \% ; \mathrm{P}<0.001$ ). When asked "What can be done to better support you?" the most frequent response was "more personal protective equipment (PPE)" (37.1\% in summer vs $26.6 \%$ in fall; $\mathrm{P}<0.001)$ and "provide more flexibility with schedules" (26.5\% in summer vs $21.9 \%$ in fall; $\mathrm{P}<0.001)$. More women than men wanted flexibility with schedules (summer: $32.5 \%$ vs $22.6 \%$, respectively; fall: $25.3 \%$ vs $18.4 \%$, respectively; $\mathrm{P}<0.001$ ) and support for mental health needs (summer: $16.9 \%$ vs $10.7 \%$, respectively; fall: $19.1 \%$ vs $11.1 \%$, respectively; $\mathrm{P}<0.001$ ).

Conclusion: Over time, in this population, the pandemic has disproportionally impacted women who reported more burnout, caregiving challenges, and desire for mental health support. The Joy of Work team worked with organizational leadership and operations teams to respond to these concerns, which may have influenced decreased rates of concern about safety, requests for more PPE, flexibility with schedules, and mental health needs.

\section{OTHER}

Saint Louis University and SSM Health Virtual Data Warehouse (VDW): Lessons Learned From the Creation of a Multi-Institutional VDW in the U.S. Midwest

\author{
Leslie Hinyard, Katie Sniffen, Jeffrey Scherrer
}

Saint Louis University / SSM Health, St. Louis, MO

Background: HCSRN membership can lead to transformative growth in research collaboration and funding. Saint Louis University (SLU) has funded the development of a Virtual Data Warehouse (VDW) containing electronic health record (Epic) data from approximately 4 million patients who have received care in SLU's academic medical practice or our partner system SSM Health. Herein, we describe the development of a unique, comprehensive VDW encompassing data from two organizationally distinct collaborative entities.

Methods: A merged instance of Epic between SSM Health and the SLUCare medical practice provided a unique opportunity for the development of an SLU-SSM Health VDW. Working collaboratively with both medical organizations, SLU researchers obtained a data use agreement (DUA) for shared data use across the institutions. Upon obtaining institutional support for the project, we recruited an experienced HCSRN VDW developer and established a phase 1 goal to extract a limited database with monthly updates.

Results: This unique academic-private health system collaboration began after DUA completion. The VDW build began in June 2019, with projected completion in March 2021, and SLU successfully applied for membership into the HCSRN in September 2020. The SLU-SSM Health VDW includes 23 hospitals, nearly 300 ambulatory care sites, and approximately 4 million patients who received care in Missouri, Illinois, Oklahoma, and Wisconsin from 2015 to 2021. Of those, 1.7 million have established primary care within SSM Health. Patient demographics include sufficient samples of African American and White urban and rural populations of low income to offer a unique resource for health disparities research. Challenges to development included identification and inclusion of key stakeholders in both organizations, conforming to data security and compliance policies at two separate organizations, timing of data transfer from SSM Health to SLU, and interorganizational collaboration during the COVID-19 pandemic.

Conclusion: The SLU-SSM Health partnerships allows for novel health services and outcomes research comparing academic to private sector delivery of care. Bridging two independent organizations with shared data resources presents both challenges and advantages during resource development. 


\section{Lessons Learned From a Pilot Study on Cognitive Behavioral Therapy for Chronic Pain: Qualitative Findings}

Dea Papajorgji-Taylor, Alison Firemark, Meghan Mayhew, Sara Gille, Lynn DeBar, Carmit McMullen

\section{Kaiser Permanente Northwest, Portland, OR}

Background: Approximately $10 \%$ of adults are affected by chronic pain, for which cognitive behavioral therapy for chronic pain (CBT-CP) is an effective and accepted alternative to pharmacotherapy treatments. Making CBT-CP widely available in medically underserved populations, in addition to delivering these services at low cost, would reduce access barriers for a significant portion of the U.S. population.

Methods: A comparative effectiveness study is currently underway in 4 integrated health systems and is evaluating the delivery of CBT-CP via 2 low-cost scalable modalities: an established web-based intervention, and a virtual coach-led intervention covering similar content. Formative research was conducted to pilot test both interventions and gather feedback for refining the trial protocol. In-depth semi-structured interviews conducted by telephone with 8 health system leaders and 7 patients explored feasibility, acceptability, alignment of interventions with existing pain management programs, and opportunities for improvement of study procedures. Thematic analysis and comparative qualitative data visualization techniques helped to synthesize results.

Results: Health system stakeholders emphasized support for the trial and anticipated valuable results from it. They anticipated likely adoption of the interventions and saw these as aligned with existing pain management initiatives. Several concrete suggestions about recruitment and information-sharing between the trial and health systems were offered. Patients in both arms reported similar motivators for enrolling in the study, including outreach through the health system, an opportunity to gain new pain management skills, having an open mind, potential for pain relief, and a desire to help others through research. Common motivators for intervention completion included dedication to the commitment made to participate and familiarity with the material presented. Both groups had mixed experiences of learning valuable skills but generally anticipated sustained skill use postintervention. Web-based intervention participants stated that convenience and flexibility supported intervention completion. They were less likely to report that the intervention met their expectations compared to participants in the coach-led intervention. Coach-led intervention participants valued engagement with trained professionals.

Conclusion: Findings resulted in modest protocol changes and confirmed that the trial design was feasible and welcome, especially for medically underserved areas. Data visualization techniques greatly enhanced communication of pilot study findings to the study team.
Adults Exposed to Tear Gas Report Physical and Psychological Health Issues and Health Care Utilization: A Cross-Sectional Survey From Portland, Oregon

Britta Torgrimson-Ojerio, Karen Mularski, Madeline Peyton, Erin Keast, Jason Scott, Ilya Ivlev

\section{Kaiser Permanente Northwest, Portland, OR}

Background: Although banned in international warfare, U.S. civilians are being subjected to chemical munitions by law-enforcement agencies. Interest in health policy on the use of these chemical agents on the general population is increasing; as of August 2020, lawmakers in 9 states had introduced bills that would ban or limit the use of tear gas on civilians. This study was designed to describe the health effects and subsequent health care utilization in civilians who were exposed to tear gas agents by law-enforcement agencies during protests of racial and social injustice in 2020 in Portland, Oregon.

Methods: We conducted a cross-sectional online survey administered July 30-August 20, 2020, in a convenience sample of 2257 adults exposed to tear gas.

Results: The majority of respondents self-identified as female/cisgender women $(\mathrm{n}=1151,51.0 \%)$ and White/ non-Hispanic $(\mathrm{n}=1615,71.6 \%)$. Of the 2257 persons who reported tear gas exposure, $2116(93.8 \%)$ reported physical $(\mathrm{n}=2114,93.7 \%)$ and/or psychological $(\mathrm{n}=1635,72.4 \%)$ health issues immediately or within days after being exposed. Most $(\mathrm{n}=2105,93.3 \%)$ reported eye $(88.4 \%)$, nose (79.3\%), lungs/chest (69.5\%), mouth (66.9\%), and/or skinrelated $(54.9 \%)$ issues immediately after exposure. Notably, we report ongoing health issues 1 to 2 days after exposure in lungs and/or chest $(n=1063,47.1 \%)$, gastrointestinal $(n=638$, $28.3 \%)$, head $(n=626,27.7 \%)$, and eye issues $(n=594$, $26.3 \%$ ). Of 1650 persons who identified as female/cisgender woman, transgender male, genderqueer (not exclusively female or male), or did not specify sex or gender identity, the majority $(\mathrm{n}=899,54.5 \%)$ reported menstrual disruption (ie, increased bleeding, abnormal spotting, increased menstrual cramping) and/or breast/chest tenderness. Most respondents, 1635 (72.4\%), reported increased anxiety, startle response, fear, fatigue, or sadness/depressive feelings after the exposure. The majority of respondents $(n=1233,54.6 \%)$ received or planned to seek health care for their physical and/or psychological tear gas-related health issues. Of the 2257 exposed to tear gas, $944(41.8 \%)$ reported receiving medical care immediately after exposure.

Conclusion: We report tear gas-related health issues affecting multiple body systems, sometimes persisting for days to weeks, and often requiring medical attention that could potentially be avoided otherwise. Our findings may further inform policy on the public health impact of using of these chemical agents on the general population. 


\section{PATIENT, CLINICIAN, AND HEALTH SYSTEM ENGAGEMENT}

\section{Using Survey Data to Assess Clinician Perspectives on Comparative Performance Feedback Interventions on Opioid Prescribing}

Zijun Shen, Farah Refai, William Isenberg, Amol Navathe, Mitesh Patel, Joshua Liao, Xiaowei (Sherry) Yan

\section{Sutter Health, Walnut Creek, CA}

Background: We surveyed 437 clinicians from emergency departments and urgent care clinics across Sutter Health on their perspectives on an email-based opioid intervention. The 4-arm randomized intervention involved sending clinicians comparative performance feedback on their opioid prescribing, and was live from September 2019 to February 2020. We analyzed survey responses to understand clinician perspectives on the email intervention and to determine the impact on clinical decision-making when prescribing opioids to patients.

Methods: A pre- and a postintervention survey were sent in April 2019 and September 2020, respectively. We compared clinicians' perspectives of comparative performance feedback before and after the intervention and assessed clinicians' feelings regarding the intervention using descriptive statistics. Results: A total of 139 clinicians responded to both pre- and postintervention surveys, compared to 167 clinicians who responded to one survey and 157 clinicians who did not respond to either. The baseline opioid prescription rate is slightly higher for nonrespondents (14.2\%) compared to those who responded to both (12\%). Compared to the preintervention survey, fewer clinicians agreed on the effectiveness of receiving comparative performance feedback on their opioid prescribing than in the postintervetnion survey ( $49 \%$ to $42 \%)$, and the perception of another clinician's prescribing practices influencing one's own prescribing behavior increased from $21 \%$ to $29 \%$. Most clinicians claimed to trust the data in the feedback email, varying by intervention arm ( $46 \%$ to $62 \%)$, and most $(67 \%$ to $76 \%$ ) also agreed that receiving the feedback via email was convenient. Clinicians in certain arms (46\% in combination and $48 \%$ in peer comparison) felt that the email intervention was a critique of their clinical decision-making compared to the individual feedback arm $(11 \% ; \mathrm{P}<0.001)$. About $30 \%$ of clinicians in the peer comparison and combination arms felt that their autonomy as a clinician was threatened by receiving the feedback email. A significant amount of clinicians $(35 \%$ in peer comparison and $30 \%$ in combination arms) reported that the email intervention impacted their opioid prescribing behavior.

Conclusion: Though evidence demonstrated that the peercomparison intervention improves clinicians' practice behavior, the clinicians' perspectives of being monitored and critiqued on their clinical decisions cannot be ignored.
Designing Educational Mailings to Motivate Patient-Caregiver-Provider Conversations About Deprescribing

Mary Antonelli, Sam Cox, Cassie Saphirak, Abir Kanaan, Jerry Gurwitz, Kathleen M. Mazor, Sonal Singh

Meyers Primary Care Institute, Worcester, MA

Background: Inappropriate prescribing includes the use of unnecessary medications or medications that increase the risk of adverse events. Sedative-hypnotic, antipsychotic, and anticholinergic agents pose risks for older adults, especially those with Alzheimer's disease and Alzheimer's disease-related dementias (AD/ADRD). Educating patients and caregivers about the potential for inappropriate prescribing and prompting them to talk with their health care provider about their medications may help stimulate deprescribing of inappropriate medications. We sought to create educational mailings for patients with $\mathrm{AD} / \mathrm{ADRD}$, with the goal of motivating the patient or their caregiver to initiate a conversation with their provider about potentially inappropriate medications.

Methods: We drafted cover letters and educational brochures for sedative-hypnotics, antipsychotics, and anticholinergics, drawing on existing tested materials. We conducted semistructured interviews with patients and caregivers via Zoom and telephone to elicit their reactions to the materials and to explore whether they would bring the materials to their next health care visit as the materials suggested. Interview transcripts were analyzed qualitatively. An advisory panel and stakeholder panel also provided feedback and suggestions. Materials were iteratively adapted and refined based on feedback from the interviewees and panelists.

Results: We conducted 14 interviews, involving 9 caregivers, 2 independent patients, and 3 patient-caregiver dyads (total $\mathrm{N}=17$ ). Interviewees were receptive to the ideas of bringing the materials to their next health care visit and initiating a conversation with their provider. Most patients indicated the materials would be shared with the caregiver. Major recommendations included: 1) keep materials specific to the patient, concise, understandable, and with clear action steps; 2) use color to highlight key information and emphasize importance; 3) align text with visuals to reinforce understanding; 4) convey warmth and empathy; and 5) be mindful that patients want to avoid offending the provider or disrupting their relationship. Final materials were more concise than the originals, with a sharper focus on medication risks and the importance of talking with the provider.

Conclusion: We successfully engaged diverse participants to create specific, concise, and easily understandable educational mailings intended to encourage patients and caregivers to engage in conversations with their provider. We are planning a small pilot mailing to evaluate feasibility of a large pragmatic trial. 
Systems Mapping to Understand Colorectal Cancer Screening Underutilization in an Integrated Health System: A Valuable Approach for Learning Health Systems

Patricia L. Mabry, Andrew R. Zinkel, Meghan Jaka, Jennafer Mee, Nathaniel D. Osgood, Jeanette Y. Ziegenfuss, Nico Pronk, Anne Book, Ann Werner, Kristen Kopski, Greg Fedio, Gary Kitching, Kody Donahue, Matt Flory, Charles R. Rogers

\section{HealthPartners, Bloomington, $M N$}

Background: Learning health systems are health systems that engage in self-study to improve quality, efficiency, and effectiveness of the care they deliver. HealthPartners, a nonprofit integrated health system in the U.S. Midwest, used systems mapping to understand barriers to member/ patient underutilization of colorectal cancer (CRC) screening and identify strategies to address these barriers. Methods: Semi-structured interviews were conducted with HealthPartners members $50+$ years of age $(n=40)$; thematic content analysis revealed barriers to CRC screening. Barriers from a recent national survey of unscreened adults 45-75 years of age $(n=1021)$ also were considered. Stakeholders within and outside of HealthPartners $(n=40)$ familiar with CRC screening constructed causal maps of the identified barriers and strategies to address them. HealthPartners members'/patients' attitudes toward barrierreduction strategies were assessed in an online survey (age range: $45-75$ years; $n=261$ ). Lastly, 14 modeling experts and 6 subject matter experts added extensive detail and logic to the barrier/strategy causal maps, transforming them into interconnected systems maps that informed the development of a dynamic simulation model (model results presented elsewhere).

Results: 1) Perceived barriers to CRC screening: invincibility to CRC; unpleasantness/fear of the prep/ test/results; lack of time/resources to get screened; and financial challenges. 2) Surveyed attitudes toward barrierreducing strategies: a majority of respondents would not be motivated by incentives (64\%); many preferred evening $(28 \%)$ or weekend $(41 \%)$ colonoscopies; and some would utilize a ride program (37\%). 3) Causal maps of barriers/ strategies and model structures and their advantages, limitations, and recommendations were delivered for use in simulation model development.

Conclusion: Multimethod, multistakeholder data collection and systems mapping provided participant stakeholders with a deeper understanding of CRC screening underutilization at HealthPartners and identified strategies to improve participation. A beneficial byproduct of the mapping process was new communication/collaboration pathways across the organization. Uncovering patient/ member opposition to incentives for CRC screening prevented implementation of a potentially flawed strategy, while discovering patient/member enthusiasm for evening/ weekend scheduling and rides led HealthPartners to reconsider these strategies. Systems mapping informed the development of a dynamic simulation model. By enabling virtual experimentation, the model can be used to systematically examine trade-offs between different CRC barrier-reducing strategies, thereby informing strategy adoption at HealthPartners. In sum, systems mapping can be a valuable tool for learning health systems.

\section{Randomized Trial of Variable Performance Feedback and Its Effects on Influenza Vaccination at Kaiser Permanente Georgia}

\section{Felipe Lobelo, Alan Bienvenida, Belkis Pimentel, Henry Eyring, Kate Koplan}

\section{Kaiser Permanente Georgia, Atlanta, GA}

Background: Provision of performance feedback to health care providers (HCPs) can improve overall rates of delivering screening and other preventive services. However, researchers have yet to examine whether targets can change individual HCP performance in health care settings. In this study, we assessed how varying performance target designs can produce improvements in the successful provision of influenza (flu) vaccinations.

Methods: Between September 23, 2019, and December 31,2019 , we conducted a randomized controlled trial to assess the effectiveness of variable performance target designs on provider flu vaccination performance, defined as the number of successful flu vaccinations over their total patients counseled (opportunities). A total of 595 HCPs at Kaiser Permanente Georgia (62.0\% female, $84.7 \%$ physicians) were enrolled and categorized into 4 groups (primary care, medical specialty, procedural \& surgical, and other) based on their specialty and randomly assigned to 1 of 3 performance target design conditions: 1) organizational control target (CT), defined as $50 \%$ success static target $(\mathrm{n}=204) ; 2)$ achievable target (AT), defined as static target 10 percentage points higher than the providers' baseline performance $(\mathrm{n}=197)$; and 3) variable target (VT), defined as a dynamic target set 10 percentage points higher than the individual best and rising another 10 points in the 2 -week period after the provider achieves it $(\mathrm{n}=194)$.

Results: Over the study period, there were 117,230 unique flu vaccination opportunities among all participating HCPs. Overall, HCPs who received VT $(22.3 \%$, 95\% CI: 20.5-24.1) or AT (21.4\%, 95\% CI: 19.5-23.2) performance feedback designs had significantly higher $(\mathrm{P}<0.05)$ average flu vaccination success rates, as compared to HCPs in the CT condition (19.5\%, 95\% CI: 17.5-21.9). When stratified by provider demographics, VT resulted in significant flu vaccination success rate improvements for younger HCPs (4.3\%; $\mathrm{P}<0.001)$, male HCPs $(1.1 \%$; $\mathrm{P}<0.05)$, and shorter- 
tenured HCPs $(3.5 \% ; \mathrm{P}<0.05)$. Additionally, HCPs who received the VT had higher flu vaccination success rates with older $(2.7 \% ; \mathrm{P}<0.05)$, White $(4.8 \% ; \mathrm{P}<0.05)$, and Black patients $(3.2 \% ; \mathrm{P}<0.05)$ compared to HCPs in the CT.

Conclusion: Alternative performance feedback designs such as variable and achievable targets led to significant improvements in flu vaccination success rates across a variety of provider and patient demographics.

\section{POPULATION HEALTH IMPROVEMENT}

\section{"I Would Never Have Done It on My Own" - Patient Satisfaction With Advanced Notifications and Reminders as Part of a Mailed Fecal Immunochemical Test Colorectal Cancer Screening Program}

Jennifer L. Schneider, Jennifer S. Rivelli, Katie Vaughn, Jamie Thompson, Amanda Petrik, Gloria Coronado

\section{Kaiser Permanente Northwest, Portland, OR}

Background: Colorectal cancer (CRC) screening by annual fecal immunochemical test (FIT) is an accessible and costeffective strategy to lower CRC incidence and mortality. Mailed FIT outreach programs have been shown to improve CRC screening rates in community health centers, with improvements ranging from $22 \%$ to $45 \%$. Despite use of primers (advanced notifications) and reminders for mailed FIT programs, less is known about patient satisfaction of these communications for motivating FIT completion.

Methods: To improve rates of CRC screening, the Participatory Research to Advance Colon Cancer Prevention (PROMPT) trial studied the effectiveness of an enhanced mailed FIT outreach program that adds primers (text messages or live phone calls) and automated phone call reminders. The program was administered at a Los Angeles-based community health center that provides medical services to 280,000 patients annually $(82 \%$ are Latinx). As part of PROMPT, we conducted telephone interviews with a subset of English-language (EL) and Spanish-language (SL) participants who experienced the enhanced mailed FIT program and primers. Our goal was to assess acceptability, helpfulness, and advice for program improvement.

Results: We completed 25 interviews $(n=13$ SL, $n=12$ EL) with 15 females and 10 males, 68\% $(n=17)$ of whom were 50-60 years of age, and 15 with no identified prior experience completing a FIT. Most patients found the frequency and content of the primers and reminders appropriate and professional. SL patients were less likely to recall receipt of primer texts, with a few indicating technology challenges. The majority of patients preferred the live call primer for increasing awareness of the importance of CRC screening and creating patient accountability for completion. This theme was endorsed more often by SL patients and those who had never completed a FIT in the past. Primer texts also were found satisfactory to patients, viewed as less intrusive than calls, and less likely to be ignored. Automated reminders, while recognized as helpful for overcoming inertia, were leastpreferred due to being impersonal and lacking clear caller identification. All participants felt the program should continue.

Conclusion: An enhanced mailed FIT program with primers and reminders is acceptable to patients and helpful for overcoming common barriers such as lack of awareness, completion confusion, and procrastination.

\section{Cardiometabolic Health Gaps: How Precisely Can Therapeutic Care Gaps Be Identified From Electronic Health Records?}

Xiaowei (Sherry) Yan, Walter Stewart, Hannah Husby, Jake Delatorre-Reimer, Satish Mudiganti, Farah Refai, Andrew Hudnut, Kevin Knobel, Karen MacDonald, Frangiscos Sifakis, J.B. Jones

\section{Sutter Health, Walnut Creek, CA}

Background: Demand is increasing for efficient, higherquality management of cardiometabolic health. Because cardiometabolic health outcomes are mediated by cliniclevel (eg, knowledge, communication) and individuallevel factors (environment, health behaviors, genetics, etc), the ability to more precisely identify the root cause of a health gap may help drive actions to close them. We sought to understand the utility of structured electronic health record (EHR) data to precisely identify the gaps in therapeutic care processes (eg, physician ordering) that underlie cardiometabolic health gaps.

Methods: Using structured EHR data (2015-2017) from 249,659 primary care patients, 35+ years of age, we identified cardiometabolic health gaps for guidelinedefined control of blood pressure, low-density lipoprotein cholesterol (LDL-C), and hemoglobin A1c, as well as corresponding guideline-based therapeutic care gaps. Logistic regression models were applied to determine if cardiometabolic health gaps were explained by 1) lack of medication retrieval or low treatment adherence, 2) lack of health gap closure by the physician, or 3) uncertain.

Results: EHR data identified cardiometabolic health gaps in $44 \%$ of individuals with hypertension, 33\% with hyperlipidemia, and $57 \%$ with diabetes. Failure to fill medication was common in hypertension $(39 \%)$, hyperlipidemia (27\%), and diabetes $(27 \%)$ but was not strongly related to cardiometabolic health gaps (odds ratios [OR] of 1.23 to 1.76). By comparison, a provider's lack of medication prescription (ie, therapeutic inertia) 
was common for hypertension (16\%), hyperlipidemia (40\%), and diabetes $(27 \%)$ and was strongly related to hyperlipidemia (OR: 5.8) and diabetes (OR: 5.7) health gaps. In 9\% (hyperlipidemia) to $21 \%$ (diabetes) of patients, we could not determine if a cardiometabolic health gap was explained by patient adherence or a physician-related therapeutic care gap.

Conclusion: Cardiometabolic health gaps and related therapeutic care gaps can be identified from structured EHR data. Therapeutic inertia is highly correlated to health gaps and could represent an actionable target for health systems. The underlying cause of persistent care gaps for hyperlipidemia and diabetes is difficult to identify in up to $20 \%$ of patients, suggesting that better structured data and/ or more sophisticated decision support is needed to help close these gaps in care settings.

\section{A Developmental Evaluation of the Screening, Treatment, and Prevention of Pediatric Adverse Childhood Experiences at Kaiser Permanente Southern California}

\section{Kelly Dumke}

\section{Kaiser Permanente Southern California, Los Angeles, CA}

Background: More than 20 years ago, Kaiser Permanente (KP) partnered with the Centers for Disease Control and Prevention and uncovered the devastating impacts that adverse childhood experiences (ACEs) can have across the life course. While frameworks for treating or preventing ACEs exist, there is limited practice-based guidance on the role of the health care sector, especially when it comes to pediatric populations. This study capitalized on $3 \mathrm{key}$ opportunities: a new California initiative seeking to cut ACEs in half in one generation (ACEs Aware); a lack of practice-based research on pediatric ACEs; and an ongoing pediatric screening pilot at KP Southern California.

Methods: A mixed-methods developmental evaluation of KP Southern California's Pediatric ACEs Pilot Program was conducted to explore 4 research aims: 1) understand the current burden of pediatric ACEs (through quantitative analysis); 2) evaluate pilot efforts from multiple perspectives, including pediatricians, nurses, social workers, and community organizations (through semi-structured interviews); 3) compare pilot efforts to recommendations for trauma-informed care (through semi-structured interviews); and 4) explore opportunities for KP's role in primary prevention (through focus group). Results: Among pediatric members screened at KP $(\mathrm{n}=8473), 1$ in 5 had an ACE score of 1+ with statistically significant differences across age, race/ethnicity, pilot site, and income. Key findings when examining perceptions of the pilot included the need for more training on traumainformed care, strengthening resources and referral networks, adjusting the questionnaire to account for cultural differences and include social determinants of health, and offering more follow-up. In exploring KP's role in the primary prevention of ACEs, key findings showed investment in multiple prevention-focused strategies, but they often focused at one medical center or one community. Conclusion: Health care has both a downstream (treatment) and upstream (prevention) role to play in addressing pediatric ACEs. From a downstream perspective, KP can use ACEs screening as a tool (not a diagnostic) but within the context of trauma/resilience-informed care. From an upstream perspective, KP can continue to collaborate across sectors, invest in key environments, and leverage data to pinpoint investment and collaboration. This developmental evaluation provides a draft blueprint for KP's role in the screening, treatment, and prevention of pediatric ACEs.

\section{Evaluation of the National Diabetes Prevention Program Among Adults With Prediabetes in the Southeastern United States, 2018-2020}

Tina Davis, Courtney McCracken, Ann Whitaker, Cristina Bozocea, Felipe Lobelo, Belkis Pimentel

\section{Kaiser Permanente Georgia, Atlanta, GA}

Background: While 34.2 million Americans have diabetes, 88 million adults in the United States have prediabetes. Without lifestyle changes, people with prediabetes have increased risk of developing type 2 diabetes, stroke, and heart disease. Lifestyle changes decrease risk of developing type 2 diabetes by as much as $58 \%$. The National Diabetes Prevention Program (National DPP) lifestyle change program is a 12-month evidence-based intervention developed in 2010 to address increasing burden of prediabetes and type 2 diabetes. Adults in National DPP were one-third less likely to develop diabetes. Kaiser Permanente Georgia (KPGA) achieved full recognition for their National DPP lifestyle change program, which is offered to members at no cost. This study is an evaluation of members from 2018 to 2020 who participated in the National DPP at KPGA.

Methods: Members were eligible to participate if they were 18 years old or older, not pregnant, not diagnosed with diabetes, currently enrolled at KPGA, and met at least 1 of the following criteria: hemoglobin A1c of 5.7-6.4, previous prediabetes or gestational diabetes diagnosis, and a score of $>5$ on the prediabetes risk test. A total of 254 members participated in National DPP during 2018-2020. The primary outcome was weight loss at 12 months postbaseline session; participants were included in the analysis of weight trajectory based on having at least 1 follow-up time point.

Results: National DPP participants at KPGA were predominately female $(85.1 \%, \mathrm{n}=217)$ and Black $(69.8 \%$, 
$\mathrm{n}=178)$. Median age at program start was 59 years, and $43.1 \%(n=110)$ had a college degree. Most participants $(79.6 \%, n=203)$ had a hemoglobin A1c recorded to confirm their prediabetes status. Among members with multiple weights $(n=191), 69 \%(n=132)$ lost more than 1 pound per month and $31 \%(n=59)$ showed a stable weight trajectory with weight change within \pm 1 pound per month. Results from the mixed model showed estimated weight loss was 1.59 pounds per month $(95 \% \mathrm{CI}: 1.33,1.88)$. The average minutes of physical activity per week was 150 minutes.

Conclusion: Members with prediabetes in the National DPP lost weight while participating in the lifestyle change program. Future research should look at a maintenance phase of weight loss and hemoglobin A1c 6-12 months after completing the program to understand longer-term program outcomes for program participants.

\section{TECHNOLOGY AND DIGITAL HEALTH}

\section{Effect of a Web-Based Medication Reconciliation Tool on Medication Discrepancies in a Primary Care Population}

Michael Gionfriddo, Vanessa Duboski, Melissa Kern, Bhumika Maddineni, Yirui Hu, Eric Wright

\section{Geisinger Research, Forty Fort, PA}

Background: Having an accurate medication list is a wellrecognized patient safety issue. Unfortunately, electronically recorded medication lists are not often reflective of actual medications used. We developed a web-based medication reconciliation tool $\left(\mathrm{MedTrue}^{\mathrm{TM}}\right)$ to facilitate and display the collection of accurate medication lists - through integration of patient-collected, electronic health record (EHR), and administrative claim medication information - within the EHR. Effectiveness of this tool in improving the accuracy of medication lists was evaluated.

Methods: We conducted a cluster-randomized trial within 6 primary care clinics within an integrated health delivery system. Sites were selected in collaboration with clinic leadership and randomized in a 1:1 fashion to intervention (MedTrue $^{\mathrm{TM}}$ ) or usual care using a random number generator. Patients were eligible for inclusion if they were seen at a participating clinic for a visit with their primary care provider, if they were 18 years of age or older, if they spoke English, and, for the intervention arm, if both the patient and a health care provider were exposed to MedTrue. After an eligible visit, patients were approached by a trained pharmacist, consent was obtained, and a best possible medication history was performed. The primary outcome was the rate of medication discrepancies, defined as a composite rate of medication additions, removals, or modifications.
Results: Overall, 224 patients were recruited and underwent a best possible medication history with the pharmacist (118 usual care, 106 MedTrue). The most common discrepancies identified were medications the patient was no longer taking, with a median of 2 per patient (interquartile range: 1-3) in usual care and a median of 1 per patient (interquartile range: 1-3) among those who used MedTrue. MedTrue use did not affect the rate of the primary composite outcome (odds ratio: $1.01,95 \% \mathrm{CI}$ : 0.38-2.70; $\mathrm{P}=0.982$ ).

Conclusion: MedTrue did not reduce the rate of medication list discrepancies among a population of patients seen in primary care.

\section{Cross-Organization Data Sharing Using MDClone Platform}

Gabriel Chodick, Tal Tova Patalon, Brosh Yinon, Ben Gross

Maccabi Institute for Health Services Research, Tel Aviv-Jaffa, Israel

Background: At present, most health care providers rely only on data from their facilities, which is many times lacking and telling a misleading story. It is clear that integration of information from different health care providers can dramatically improve patient quality of care, research, organization financial savings, etc.

Methods: MDClone platform is a Big Data system for health care, enabling broad use of data inside and across health systems to rapidly deliver information to improve patients' health. During the COVID-19 pandemic, MDClone platform is used to share data between two leading health care providers in Israel: Maccabi, the second-largest health management organization (HMO) in Israel $(\sim 30 \%$ of Israel population) and Sheba Medical Center, the biggest governmental hospital in Israel. As part of this collaboration, both organizations shared data on a daily/weekly basis, enabling researchers to draw a complete picture of COVID-19 continuum of care and statistics.

Results: Full historical data of 800 Maccabi COVID-19 patients that were admitted to emergency department or hospitalized in Sheba was shared between the two organizations. The vast data-sharing allowed both organizations to work on varied research. Two papers are already published, and recently the data collaboration was extended to include vaccination data, as Israel becomes the world leader, allowing the identification of side effects and long-term outcomes.

Conclusion: Following the success of this pilot collaboration, we now aim to extend this collaboration to additional areas such as oncology, neurology, and obstetrics data. 
Engaging Parents and Health Care Providers to Inform Development of a Behavioral Intervention Technology to Promote Pediatric Behavioral Health: Mixed-Methods Study

Sean M. O'Dell, Heidi R. Fisher, Victoria Schlieder, Tracey Klinger, Rachel Kininger, McKenna Cosottile, Stacey Cummings, Kathy DeHart

\section{Geisinger Research, Danville, PA}

Background: Behavioral intervention technologies (BITs) hold potential to transform primary care behavioral health by empowering parents to take charge of their child's behavioral health care. However, research is needed to understand parental needs in a BIT as well as current parent self-help practices and parent-provider collaboration to identify and address behavioral health challenges.

Methods: We conducted a convergence-validation mixedmethods study in which parent quantitative surveys $(\mathrm{N}=385)$ on preferences and current practices related to behavioral health themes to be addressed in a BIT were integrated with focus group interview data on internal and external contextual factors contributing to parental unmet needs and current practices with 48 health care stakeholders in 9 child-serving clinics within a large, predominantly rural health system. We integrated these data using joint displays and synthesized areas of confirmation, expansion, and discordance between parents and health care stakeholders.

Results: Parents frequently endorsed about half of the available themes, indicating that BITs may not be the preferred modality for all targeted prevention. Additionally, parents frequently endorsed themes that were not related to child psychopathology (eg, parenting stress and family communication), indicating parents are interested in guidance on parenting beyond challenging child behavior. Health care stakeholders indicated that an online platform aligns with how parents already seek behavioral health guidance and suggested that a BIT may connect families with evidence-based guidance sooner. We identified areas of convergence related to overt behavior problems and areas of divergence related to internalizing problems and cross-cutting issues that may be more difficult for health care providers to detect. Data integration expanded our understanding of factors enabling effective parent-provider partnerships, including the impact of time pressure during office visits and insights on how parenting stress influences parent help-seeking behavior.

Conclusion: These findings provide a rich understanding of the complexity involved in meeting parents' needs for behavioral health guidance in a primary care setting using BITs. Further triangulation of these findings in usertesting studies for BIT prototypes is needed to refine our understanding of how to successfully develop and implement an effective BIT to guide parents in taking charge of their child's behavioral health care.
Pulse Rate Variability as a Digital Biomarker of Low-Grade Inflammation and Its Regulation

\section{Adam Anderson}

NeurodigitX, Syracuse, NY

Background: While acute inflammation is critical to survival, chronic low-grade inflammation (LGI) is now appreciated to be a major factor in mental and physical disease, with potential onset in early development. While acute inflammation is assessed from blood samples (eg, C-reactive protein), LGI status requires repeated assessments to characterize its longitudinal evolution. Herein, we examine the use of pulse rate variability (PRV) as a noninvasive digital biomarker of LGI correlates in the brain and body.

Methods: LGI is controlled by the brain through the vagal nerve (cranial nNerve $\mathrm{X}$ ) and is 1 ) associated with older age, 2) associated with lower neuroplasticity, and 3) regulated by the vagal anti-inflammation reflex. In 65 adults across the lifespan, we assessed these 3 factors and their relation to the variability in timing between heart beats (3-lead electrocardiogram) and fingertip pulse (photoplethysmography). All participants performed measures of cognitive plasticity (learning and cognitive flexibility) and 2 minutes of an autonomic vagal maneuver (slow breathing at $0.1 \mathrm{~Hz}, 6$ breaths per minute) to engage the vagal anti-inflammation response.

Results: Consistent with LGI, lower baseline PRV was reliably associated with age, lower cognitive functional status (including decreased learning), and cognitive flexibility. Consistent with an anti-inflammation response, $0.1 \mathrm{~Hz}$ breathing transiently enhanced PRV. This was greatest for those with lower baseline PRV and thus higher LGI, lowering PRV indices of LGI equivalent to a reduction of approximately 30 years. Finally, PRV was able to capture vagal influences on heart contractions equal to electrocardiogram.

Conclusion: Acute and unresolved LGI is a critical feature of COVID-19 and its potential long-term consequences, and it is known to contribute to future noncommunicable disease burden in the young. We present evidence that PRV, by assessing and regulating vagal control of inflammation, captures critical associations between LGI with mental and physical function. PRV may serve as a noninvasive digital biomarker of the evolution of LGI and its resolution. 


\section{Patient Considerations When Selecting Telemedicine Versus In-Office Care: A Qualitative Study}

John W. Morgan, Mandy K. Salmon, Maya Ambady, Kirstin A. Manges, Corrine M. Rhodes, Nwamaka Eneanya, Judy Shea, David Grande

\section{University of Pennsylvania, Philadelphia, PA}

Background: The COVID-19 pandemic has dramatically increased adoption of telemedicine. However, how patients choose between in-office and telemedicine visit modalities remains largely unknown. Given the impact of patient choice on use of and access to telemedicine, we sought to understand how high-risk primary care patients choose between in-office, synchronous audiovisual, or audio-only telemedicine.

Methods: Between July 13, 2020, and September 30, 2020, we interviewed 29 high-risk patients who had been invited to schedule primary care visits for chronic disease management via in-office, audiovisual, or audio-only visits. Semi-structured interviews were conducted over phone. Using constant comparison, enabled by NVivo 12.1, we utilized a grounded theory approach to explore factors influencing participants' selection of visit type.

Results: Participants were predominantly female (55.2\%), Black (65.5\%), low-income (85.7\% with household income of $<\$ 50,000$ ), and elderly (mean age of $64.6 \pm 11$ years). Among interviewees, $43 \%$ had had a prior video visit. Reasons for choice of visit type were compiled into a comprehensive decision framework comprising the following factors: 1) perceptions of utility, 2) perceptions of costs, 3) choice modifiers, and 4) underlying drivers. Patients were especially sensitive to office visits' perceived superiority at revealing unexpected medical problems. Despite lower transportation "costs" with telemedicine, these were often offset by reduced ease of task-clustering (ie, inability to complete labwork or schedule appointments subsequent to the televisit). Patients' particular clinical situations, awareness of choice, and behavioral phenotype (risk aversion, assertiveness) modified decisions irrespective of cost/benefit assessments. Underlying drivers comprised global life experiences and experience with local telehealth environments.

Conclusion: Our results stand to aid health systems seeking to understand, anticipate, and inform telemedicine use. For example, by leveraging choice-framing and replicating in-person remote task-clustering efficiencies (ie, nurses in virtual "waiting rooms" prior to visit), health systems can affect telehealth utilization more effectively than by exclusively addressing low technology literacy and lack of access to technology or by focusing on more immutable factors such as distrust. The underlying drivers we identified highlight telemedicine's risk of exacerbating disparities in access and underscore the importance of alternative methods of engaging high-risk patients, including using telephoneonly services and bringing conveniences of telemedicine to in-office care (ie, direct phone lines).

(C) 2021 Advocate Aurora Health, Inc. 